\title{
Notch1 mutation drives clonal expansion in normal esophageal epithelium but impairs tumor growth
}

Emilie Abby ${ }^{1}$, Stefan C Dentro ${ }^{1,2}$, Michael W J Hall11,3, Joanna C Fowler ${ }^{1}$, Swee Hoe Ong $^{1}$, Roshan Sood ${ }^{1}$, Christian W Siebel ${ }^{4}$, Moritz Gerstung ${ }^{3}$, Benjamin A Hall ${ }^{5}$ and Philip H Jones ${ }^{1,3}$.

\section{Affiliations:}

${ }^{1}$ Wellcome Sanger Institute, Hinxton CB10 1SA, UK.

${ }^{2}$ European Molecular Biology Laboratory, European Bioinformatics Institute, Cambridge CB10 1SD, UK.

${ }^{3}$ MRC Cancer Unit, University of Cambridge, Hutchison-MRC Research Centre, Cambridge Biomedical Campus, Cambridge CB2 OXZ, UK.

4 Department of Discovery Oncology, Genentech, South San Francisco, CA, USA

${ }^{5}$ Department of Medical Physics and Biomedical Engineering, University College London, Gower Street, London WC1E 6BT, UK.

* Correspondence to: pj3@sanger.ac.uk 


\section{Summary}

NOTCH1 mutant clones occupy the majority of normal human esophagus by middle age, but are comparatively rare in esophageal cancers, suggesting NOTCH1 mutations may promote clonal expansion but impede carcinogenesis ${ }^{1-3}$. Here we test this hypothesis. Visualizing and sequencing NOTCH1 mutant clones in aging normal human esophagus reveals frequent biallelic mutations that block NOTCH1 signaling. In mouse esophagus, heterozygous Notch1 mutation confers a competitive advantage over wild type cells, an effect enhanced by loss of the second allele. Notch1 loss alters transcription but has minimal effects on epithelial structure and cell dynamics. In a carcinogenesis model, Notch1 mutations were less prevalent in tumors than normal epithelium. Deletion of Notch1 reduced tumor growth, an effect recapitulated by anti-NOTCH1 antibody treatment. We conclude that Notch1 mutations in normal epithelium are beneficial as wild type Notch1 promotes tumor expansion. NOTCH1 blockade may have therapeutic potential in preventing esophageal squamous cancer.

\section{Main text}

As our tissues age they accumulate numerous somatic mutations ${ }^{1,2,4,5}$. Disruption of some genes confers a competitive advantage to mutant progenitor cells, which may go on to form mutant clones that spread through normal tissue. Such clonal expansions are often associated with genes recurrently mutated in cancer and are considered to be a first step in malignant transformation ${ }^{2}$. However, in the esophagus, the under-representation of NOTCH1 mutants in esophageal cancer compared with normal aging epithelium suggests $\mathrm{NOTCH} 1$ mutations may even protect against malignant transformation.

The NOTCH pathway regulates cell fate in development, tissue homeostasis and cancer ${ }^{6,7}$. NOTCH1 is a cell surface receptor composed of a ligand-binding extracellular domain (NEC) and a transmembrane and cytoplasmic subunit (NTM), interacting non-covalently through the negative regulatory region (NRR) (Extended data Fig. 1a). The NRR is composed of three Lin12-Notch repeats (LNR) and a heterodimerization domain (HD) that inhibits receptor activation in the absence of a ligand ${ }^{8}$. Binding of ligands expressed on adjacent cells to conserved epidermal growth factor (EGF) repeats in the NEC results in proteolytic cleavage events that release the cytoplasmic domain (NICD), which then migrates to the nucleus and alters the expression of target genes ${ }^{8}$. In the esophagus NOTCH1 protein is expressed in the proliferating cell compartment and regulates both development and adult tissue maintenance (Extended data Fig. 1a,b) ${ }^{9}$.

The role(s) of NOTCH1 in esophageal carcinogenesis are controversial with different studies arguing NOTCH1 is a tumor suppressor or promotes carcinogenesis ${ }^{1,2,10-12}$. Here we investigate how NOTCH1 mutants take over the epithelium, the impact this has on transformation and whether NOTCH1 has potential as a therapeutic target in esophageal carcinogenesis ${ }^{1,2}$.

\section{NOTCH1 mutant clones in human esophagus}

Deep targeted sequencing studies have revealed numerous NOTCH1 mutants in human esophagus but are unable to visualize clones within the tissue and resolve which NOTCH1 mutation(s) or copy number alterations occur within each clone ${ }^{1}$. To address these issues, histological sections of normal epithelium from middle-age and elderly donors were immunostained for NOTCH1 revealing 
continuous regions of different intensity, alternating with negative areas (Fig. 1a). To determine whether this staining pattern reflected NOTCH1 mutations, we immunostained histological sections from 3 donors, aged 57, 68 and 76 years for NOTCH1 micro-dissected positive or negative areas and performed targeted sequencing for 322 genes associated with cancer (Fig. 1b). 176 protein altering somatic mutations were identified across 60 samples. Consistent with previous studies, the predominant mutations were in NOTCH1, TP53 and NOTCH2. Protein altering mutations in NOTCH1 represented a third of all mutation calls ${ }^{1,2}$ (Supplementary tables $\mathbf{1}, \mathbf{2}$, Supplementary Note). NOTCH1 mutations were near clonal with an average variant allele frequency (VAF) of 0.42 (Fig. 1c). $94 \%$ $(15 / 16)$ of negative staining areas in the two oldest donors carried nonsense or essential splice mutations or indels in NOTCH1 with copy neutral loss of heterozygosity (CNLOH) of the NOTCH1 locus (human GRCh37- chr9:139,388,896-139,440,238) or a further mutation, likely to disrupt the second NOTCH1 allele ${ }^{1}$. Of the positive staining samples, $45 \%$ (20/44) carried a missense NOTCH1 mutation and most of these had either CNLOH or a second mutation (Fig. 1c, Extended data Fig. 1c-d, Supplementary Table 2). To test if the mutations disrupted NOTCH1 function we stained consecutive sections from additional donors aged 22 to 77 years old for NOTCH1 protein and NICD1, which is detectable in the nucleus during active signaling with an anti-NICD1 antibody targeting a neo-epitope revealed after cleavage (Fig. 1d, e, Extended data Fig. 1a, Supplementary table 3). The proportion of epithelium with active NOTCH1 decreased with age (Kendall's tau- $b=-0.67, p=0.014$ ). In older donors in whom NOTCH1 mutations are common, we confirmed that NOTCH1 negative areas were associated with NICD1 loss. We also found occasional NOTCH1 positive NICD1 negative areas, consistent with the presence of missense mutant proteins that reach the cell membrane but have no detectable signaling activity (Fig. 1d, e). NICD1 positive and negative areas were histologically undistinguishable, with no significant differences in tissue thickness, cell density or the expression of the proliferation marker MKI67 (Extended data Fig. 1e-h). We conclude that the majority of NOTCH1 mutant clones in aging human esophagus carry biallelic alterations that disrupt protein expression and/or function. 


\section{Figure 1}

a.

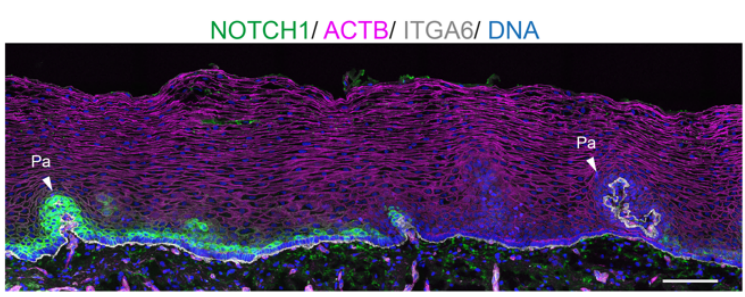

b.

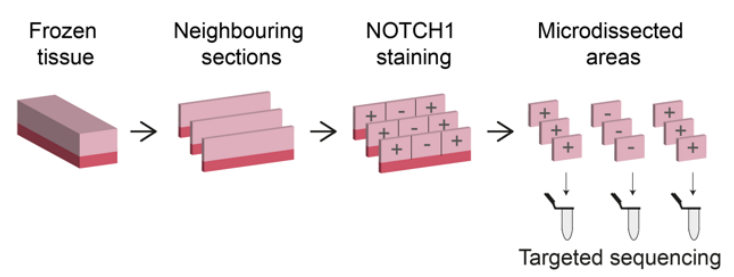

c.

d.

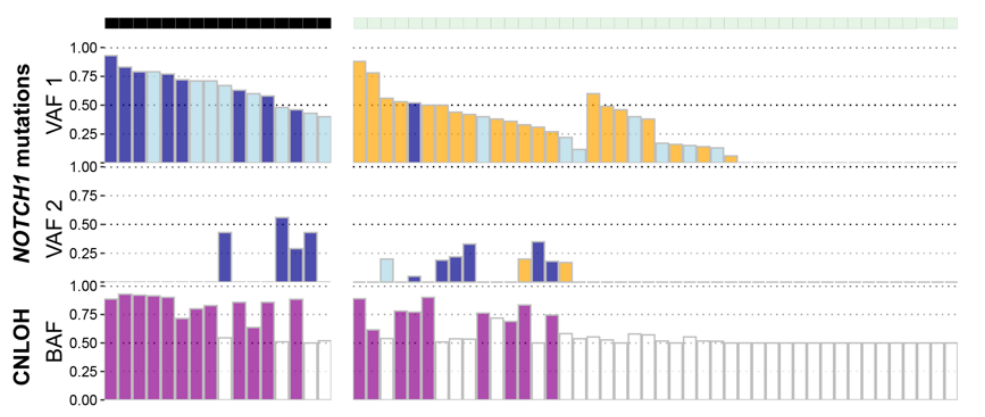

e.

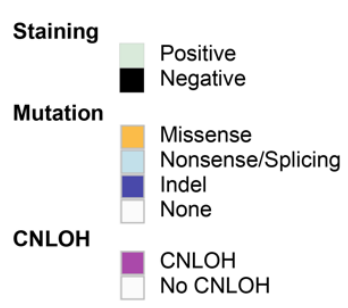

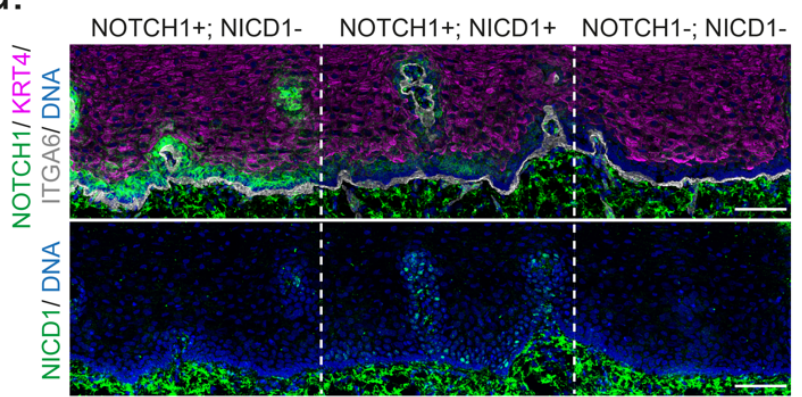

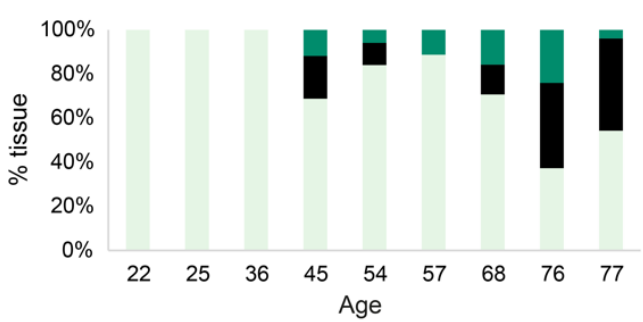

NOTCH1+; NICD1+ $\square$ NOTCH1+; NICD1- 1 NOTCH1-; NICD1-

\section{Figure 1: NOTCH1 mutant clones in human esophageal epithelium}

a. NOTCH1 (green) is expressed in the basal cell layer marked with ITGA6 (grey) but expression is lost in adjoining regions of esophagus. F-actin is magenta. $\mathrm{Pa}$, papillae. Image is representative of sections from 3 elderly donors. Scale bar, $100 \mu \mathrm{m}$. b. Protocol for $\mathbf{c}$. Frozen esophagus from three donors were sectioned and stained for NOTCH1. Contiguous NOTCH1 positive $(+)$ and negative $(-)$ staining areas from successive sections were micro-dissected and processed for targeted sequencing. c. Results from b, showing NOTCH1 staining, NOTCH1 mutation calling and copy number calling on chromosome 9. CNLOH, copy neutral loss of heterozygosity ( $n=60$ samples from 3 donors aged 57,68 and 76 years). d. Representative areas stained for NOTCH1 and NICD1 on successive sections of esophageal epithelium from an aged donor. Picture is representative of 6 middle-aged and elderly donors. Scale bar, $100 \mu \mathrm{m}$. e. Proportion of tissue stained for NOTCH1 and associated NICD1 quantified in patients aged 22 to 77 years (total section length $4774-17988 \mu \mathrm{m}$ per donor, $\mathrm{n}=9$ donors). NOTCH1+; NICD1+ areas decrease significantly with age (Kendall's tau-b correlation $=-0.67, p=0.014$ ). See Supplementary tables 1-3. 
bioRxiv preprint doi: https://doi.org/10.1101/2021.06.18.448956; this version posted June 18, 2021. The copyright holder for this preprint

(which was not certified by peer review) is the author/funder, who has granted bioRxiv a license to display the preprint in perpetuity. It is made available under aCC-BY 4.0 International license.

\section{Extended Data Fig. 1}

a.

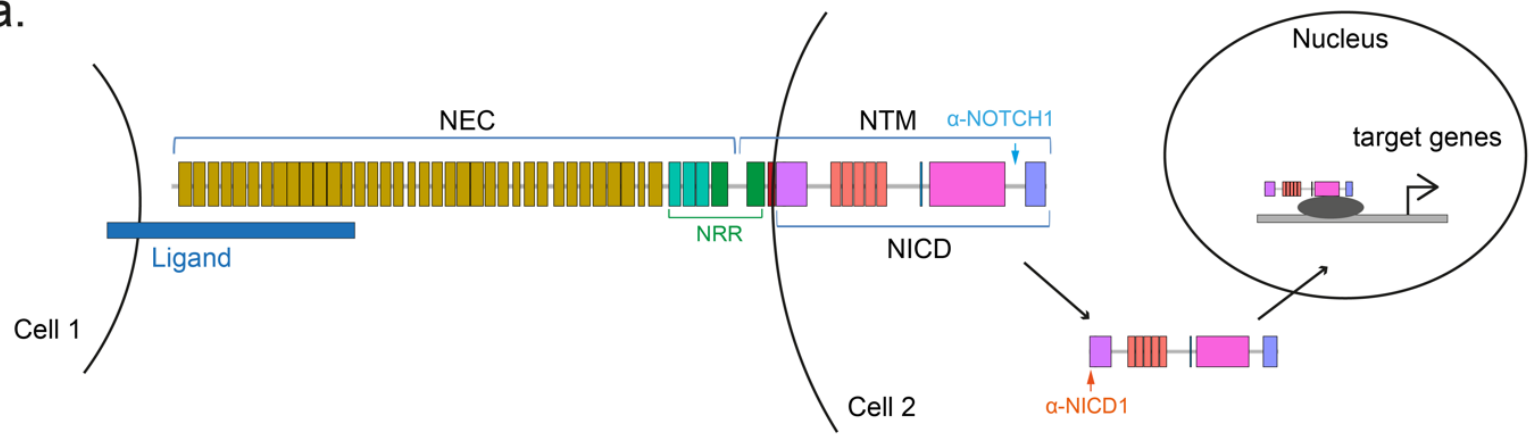

b.

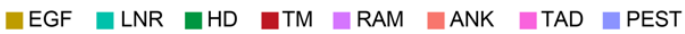

.

\section{Differentiated cell \\ - Suprabasal cell \\ (0) Basal cell}

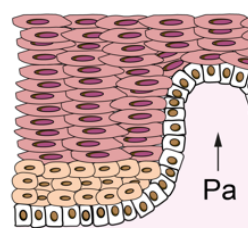

Submucosa

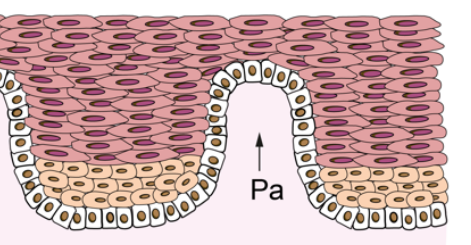

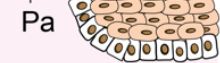

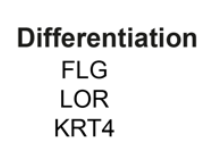

Proliferation

KRT14

ITGA6 c.

PD31008bd

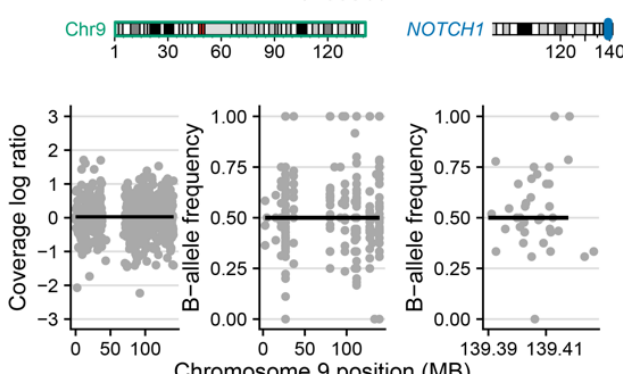

e.

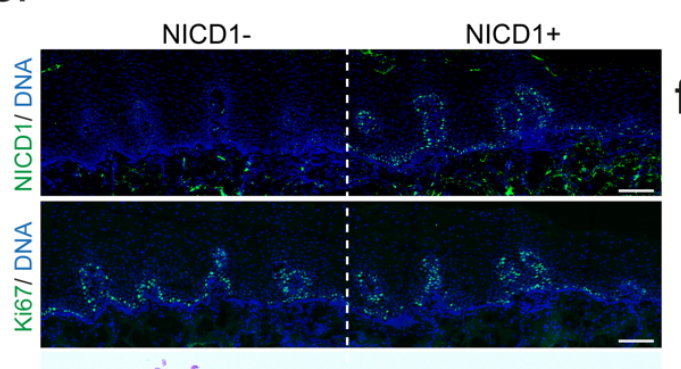

H\&E

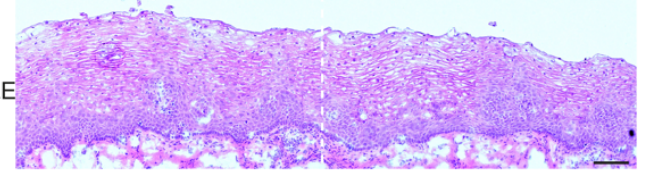

d.
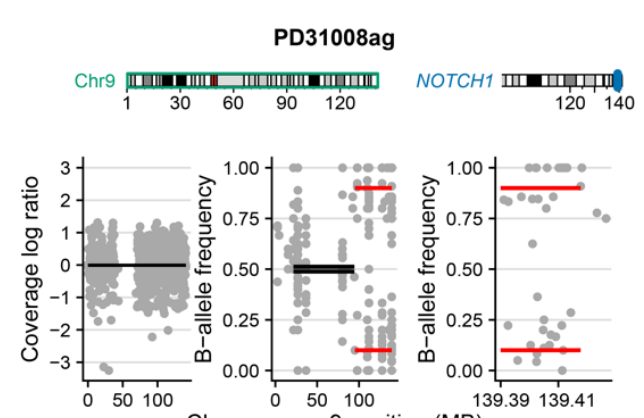

${ }_{120} 140$
Chromosome 9 position (MB)

g.

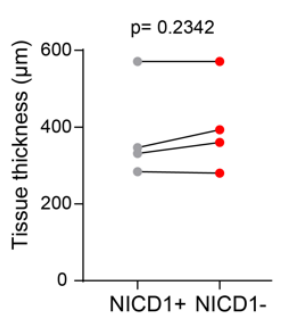

h.

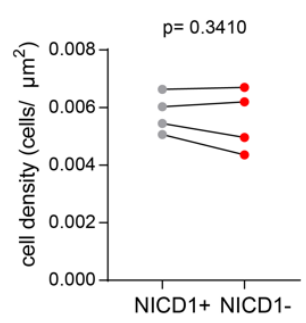

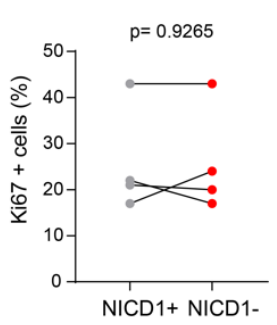




\section{Extended data Figure 1: Aging human esophageal epithelium is colonized by NOTCH1 mutant clones}

a. Schematic showing Notch signaling and NOTCH1 subunits. NOTCH1 is a membrane receptor composed of an extra-cellular domain (NEC) and a transmembrane and cytoplasmic unit (NTM). Activation by ligand expressed on a neighboring cell leads to two successive cleavages releasing the intracellular domain (NICD) which translocates into the nucleus and interacts with partners to activate target genes. Functional domains of NOTCH1 are indicated, arrows show epitopes recognized by the antibodies used for anti-NOTCH1 (blue) and anti-NICD1 (orange) immunodetection b. Human esophageal epithelium. The basal layer expresses ITGA6 and KRT14. Proliferating cells are found in the basal and lower suprabasal cell layers which express KRT14. Differentiating cells leave the proliferative compartment and migrate to the tissue surface, expressing the early and late differentiation markers KRT4 and $L O R, F L G$ respectively. Cells are continually shed from the epithelial surface and replaced by proliferation in the lower cell layers. Pa, papillae. c, d. Figures of copy number calls for the Human microbiopsies shown in Fig.1b, c and Supplementary table 2c. On the left plot, analysis of off-target reads show no difference in total copy number along chromosome 9 for both $c$ and $d$. Middle and right hand plots show the B allele frequency (BAF) of germline heterozygous SNPs along chromosome 9 and the NOTCH1 locus, respectively. Red lines denote a significant difference compared to a matched control. The figures in $\mathbf{d}$ show a high BAF for sample PD31008ag without a change in total copy number reflecting copy neutral loss of heterozygosity. e. Successive sections of esophagus from middle aged and elderly donors were stained for active NOTCH1 (NICD1), proliferation marker Ki67 and with Hematoxylin and eosin (H\&E). Images are representative from transversal sections analyzed in 4 middle-age and elderly donors. Scale bars, $100 \mu \mathrm{m} . \mathbf{f}, \mathbf{g}, \mathbf{h}$. Tissue thickness (f), cell density $(\mathbf{g})$ and proportion of proliferative cells $(\mathbf{h})$ were measured in NICD1 positive and negative areas, on sections stained for NICD1, Ki67 and DAPI. Each dot represents a donor. For $f$, NICD1+, $n=14$ areas from 4 donors, NICD1-, $n=15$ areas from 4 donors. For $\mathbf{g}$, NICD1+: 10795 cells from 4 donors, NICD1-: 11593 cells form 4 donors. For h, NICD1+: 5402 cells from 4 donors, NICD1-: 6204 cells form 4 donors. Two-tailed paired Student's t-test. See Supplementary tables 1-3. 


\section{Notch1 mutation increases clonal fitness}

To investigate how NOTCH1 mutant clones colonize normal epithelium we tracked the fate of Notch1 mutant clones in transgenic mice using lineage tracing. Mouse esophageal epithelium consists of layers of keratinocytes, with progenitor cells residing in the basal cell layer (Extended data Fig. 2a). Dividing progenitors generate either two progenitor daughters that remain in the basal layer, two differentiating daughters or one cell of each type. Differentiating cells exit the cell cycle, exit the basal layer and migrate towards the epithelial surface where they are shed. In wild type tissue, the probabilities of each progenitor outcome are balanced, generating equal proportions of progenitor and differentiated cells, maintaining cellular homeostasis across the progenitor population (Extended data Fig. 2a) ${ }^{13,14}$. Mutations can alter the balance of progenitor fate leading to excessive production of progenitors driving the growth of mutant clones ${ }^{15,16}$.

To follow the behavior of Notch1 mutant clones we generated AhCre ${ }^{E R T}$ Rosa2 $6^{\text {floxedYFP }}$ Notch ${ }^{\text {flox }}$ triple transgenic (YFPCreNotch1) mice, a conditional Notch1 model crossed with a genetic labeling system. A drug inducible form of Cre recombinase $\left(A h C r e^{E R T}\right)$ was used to delete one or both conditional Notch1 alleles in Notch $1^{\text {flox/wt }}$ or Notch $1^{\text {flox/flox }}$ animals and induce a separate conditional yellow fluorescent protein reporter allele (Rosa26 floxedYFP) so YFP was expressed in recombined epithelial cells and their progeny (Extended data Fig. 2b,c ) $^{13,17}$. This model could be induced at low dose to allow recombination of in scattered single esophageal basal cells (clonal induction) or at a high level so a larger proportion of the esophageal cells was recombined (high induction) (Extended data Fig. 2c,d).

\section{Extended data figure 2: Lineage tracing of Notch1 mutant cells in mouse oesophageal epithelium}

a. Structure and cellular homeostasis in mouse esophageal epithelium. The basal layer contains progenitor cells that divide to generate progenitor and differentiating daughter cells. Differentiating basal layer cells exit the cell cycle and migrate into the suprabasal layers, moving towards the surface of the epithelium from which they are shed. The division of a progenitor cell (green) produces two progenitors, two differentiating cells or one cell of each type. In homeostatic tissue, the likelihood of each division outcome is balanced and gives on average $50 \%$ of progenitors and $50 \%$ of differentiating cells across the progenitor population. b. YFPCreNotch1 conditional knock-out mouse strain. LoxP sites (grey arrows) flank exon1 of the Notch1 gene. Notch1 floxed animals were crossed with Rosa26 floxedYFP mice carrying a conditional Yellow fluorescent protein (YFP) reporter targeted to the Rosa26 locus and with $A h C r e^{E R T}$ mice carrying an inducible Cre recombinase. c. For lineage tracing, triple mutant mice were treated with inducing drugs at a dose that resulted in recombination of Notch1 (blue), expression of YFP (green) or both (orange) in scattered individual esophageal basal cells (clonal induction). The recombined cells may expand into clones detected by the reduced intensity $(+/-)$ or absence of NOTCH1 (-/-) and expression of YFP detected by immunostaining. Samples were collected at different time points after induction and the number and location of cells in each clone determined by 3D confocal imaging of sheets of epithelium. $\mathbf{d}$. Triple mutant mice were induced with a high dose of drugs, allowing recombination of cells at high density in the tissue. In the case of mutant clones with a competitive advantage over wild type cells, this protocol allowed the coverage of the tissue by mutant clones relatively shortly after induction. 
bioRxiv preprint doi: https://doi.org/10.1101/2021.06.18.448956; this version posted June 18, 2021. The copyright holder for this preprint

(which was not certified by peer review) is the author/funder, who has granted bioRxiv a license to display the preprint in perpetuity. It is made available under aCC-BY 4.0 International license.

\section{Extended Data Figure 2}

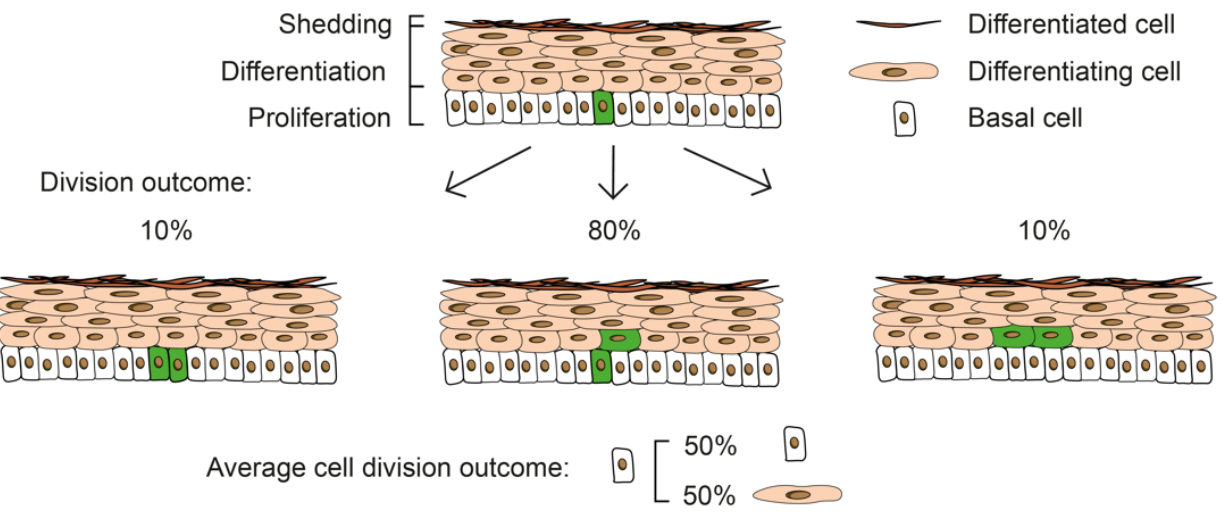

a.

b.
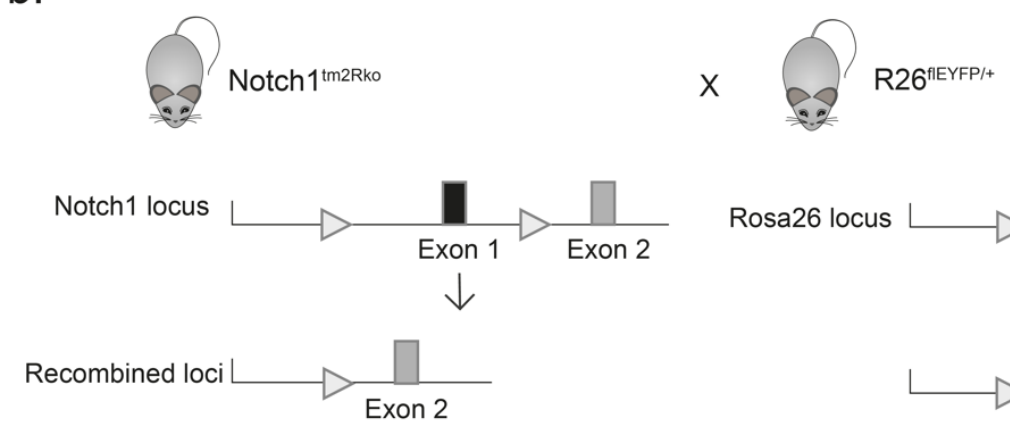

C.

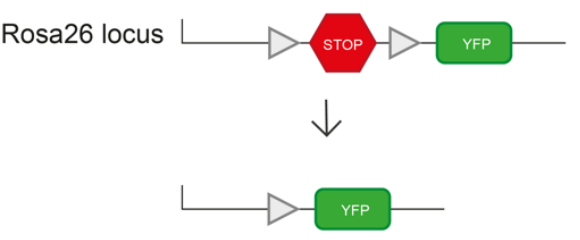

YFPCreNotch1+/+

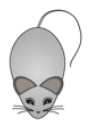

Clonal induction

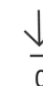

Lateral view
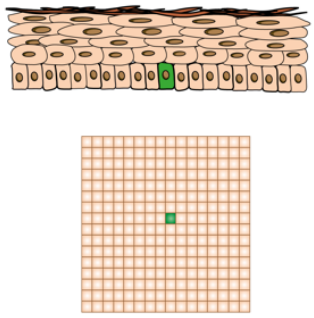

d.

YFPCreNotch1+/+

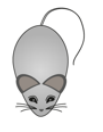

High induction

$\downarrow$

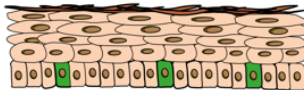

Lateral view

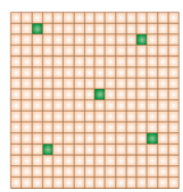

Top down view
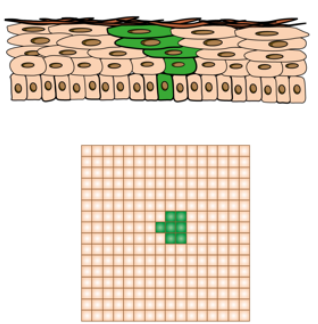

YFPCreNotch1+fllox or floxfllox

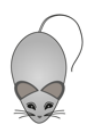

Clonal induction
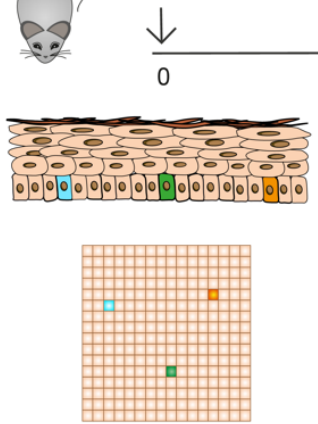
AhCre ${ }^{\text {ERT }}$
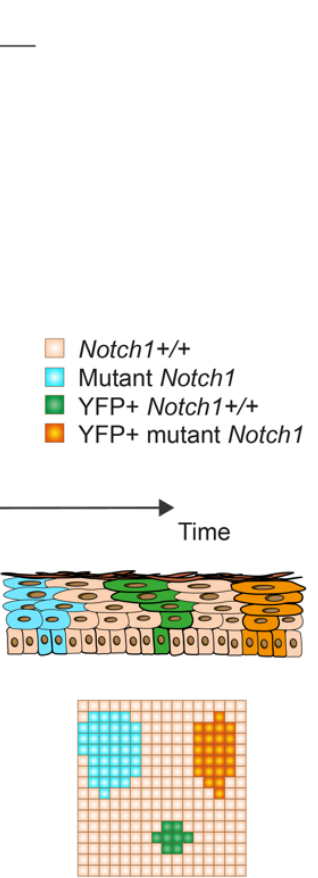

Notch1+/+

- Mutant Notch1

- YFP+ Notch1+/+

YFPCreNotch 1+/flox or flox/flox

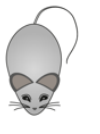

High induction

I YFP+ mutant Notch1

$\downarrow$
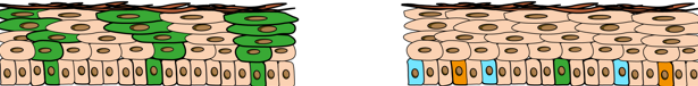

. 00100001000000010100
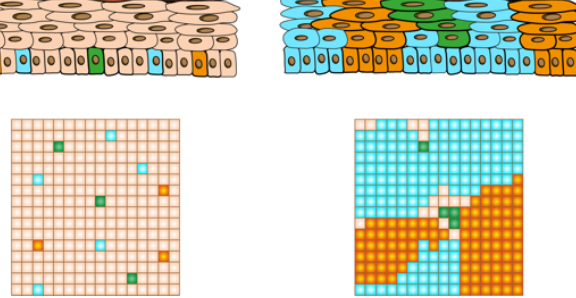
Excision of the Notch1 allele and expression of the YFP reporter at the Rosa26 locus can occur in in combination or separately, resulting in four possible populations of cells, Notch1 mutant or wild type and YFP + or - (Extended data Fig. 2c,d). To identify induced Notch1 mutant clones, we first analyzed the gene expression in wild type, Notch1 ${ }^{+-}$and Notch $1^{-}$esophageal cells finding both Notch1 mRNA and protein expression were halved in Notch $1^{+/-}$compared with wild type keratinocytes and were abolished in Notch $1^{\%}$ cells (Fig.2 a-c, Extended data 3a-e).

We then performed genetic lineage tracing by inducing recombination in scattered single progenitor cells in control $\left(\mathrm{Notch}^{+/+}\right.$), heterozygous $\left(\mathrm{Notch}^{+/ \text {flox }}\right)$ and homozygous (Notch1 flox / flox $)$ mutant animals. The growth of the YFP expressing clones in each mouse was detected by imaging sheets of epithelium stained for both YFP and NOTCH1 at multiple time points. We were able to identify YFP+ Notch $1^{+/}$and $\mathrm{YFP}+$ Notch $1^{-}$clones from the intensity of NOTCH1 immunostaining (Fig. 2d,e, Extended data Fig. 3f-i). The number and location of cells in YFP expressing clones of each genotype was then scored across the time course using 3D confocal imaging.

The size of YFP+ Notch1 ${ }^{+/}$clones was significantly increased compared with those of wild type YFP+ Notch $1^{+/+}$clones at all time points. YFP+Notch ${ }^{-/}$clones were larger still (Fig. 2e,f, Extended data Fig. 3j-k). To examine the cellular mechanisms underlying mutant clonal expansion we used short term tracking by labelling cycling cells with the $S$ phase probe EdU, and subsequently imaging the distribution of their EdU positive progeny. One hour after labelling the proportion of EdU positive cells was similar in wild type, Notch $1^{+-}$and Notch $1^{\%}$ cells, arguing the rate of cell division was similar for progenitors of all three genotypes (Fig. $\mathbf{2 g - j}$ ). At 48 hours after labelling, the proportion of suprabasal EdU positive cells reflects the rate at which basal cells differentiate and stratify out of the basal cell layer. This was significantly decreased within YFP+Notch $1^{+-}$and YFP+Notch1-- clones compared with wild type cells distant from the clone. However, in wild type cells immediately adjacent to YFP+ Notch1 ${ }^{-}$cells the proportion of EdU positive suprabasal cells was significantly increased, consistent with previous reports that Notch-inhibited keratinocytes promote the differentiation of adjacent wild type cells (Fig. $2 \mathbf{k}-\mathbf{n}$ ) ${ }^{16,18}$. 
bioRxiv preprint doi: https://doi.org/10.1101/2021.06.18.448956; this version posted June 18, 2021. The copyright holder for this preprint

(which was not certified by peer review) is the author/funder, who has granted bioRxiv a license to display the preprint in perpetuity. It is made available under aCC-BY 4.0 International license.

\section{Figure 2}

a.

YFPCreNotch1

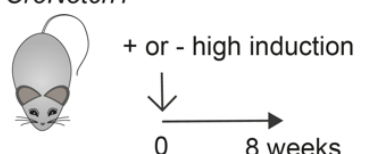

b.

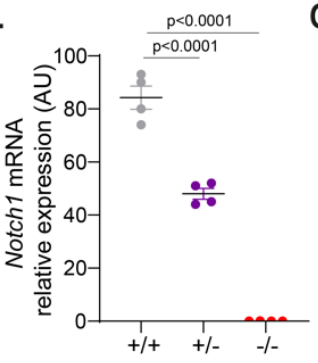

C.
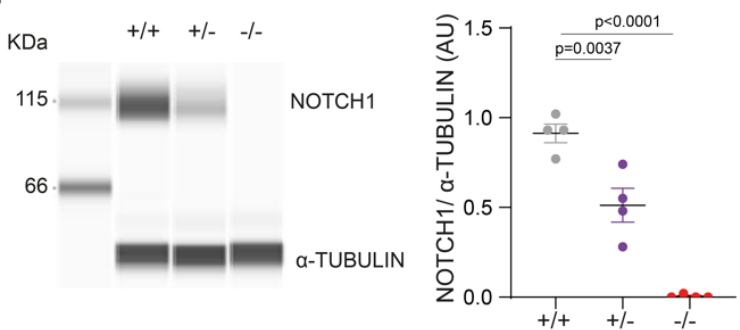

d.

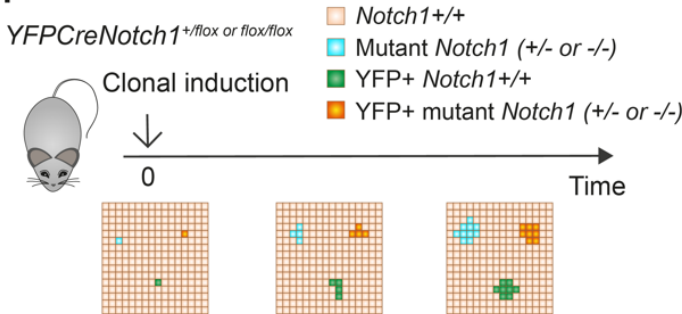

g.

YFPCreNotch $1^{\text {flloxfllox or }+ \text { fflox }}$
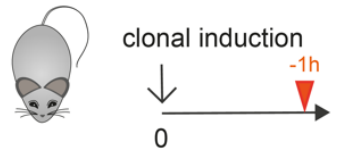

h.

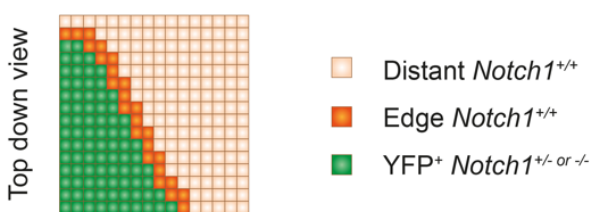

$\mathrm{k}$.

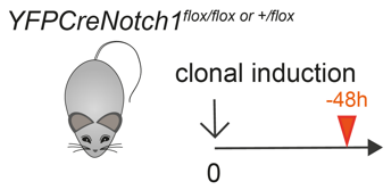

I.

$\mathrm{YFP}^{+}$Notch $^{+}{ }^{+}$

$\mathrm{YFP}^{+}$Notch $1 \%$

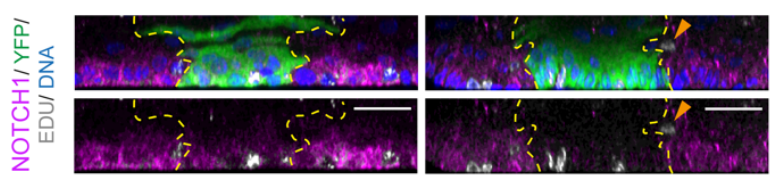

e.

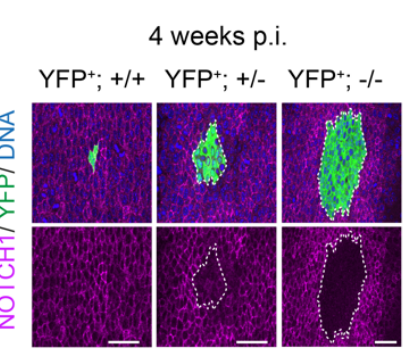

i.

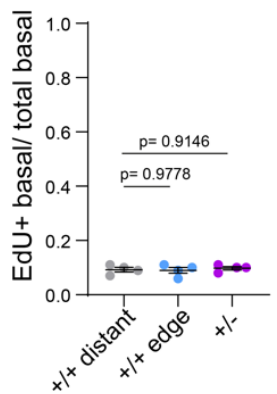

m.

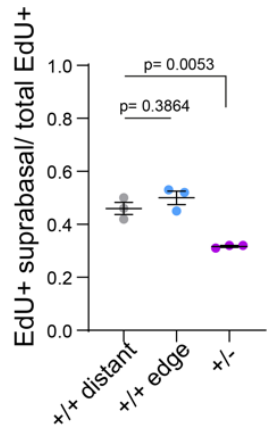

f.

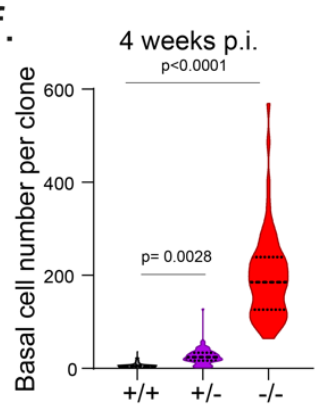

j.

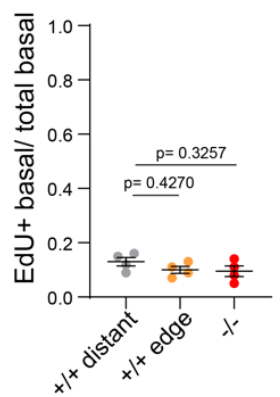

$\mathrm{n}$.

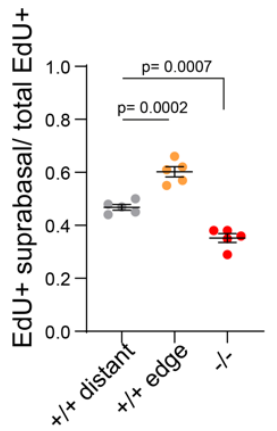




\section{Figure 2: Lineage tracing of Notch1 mutant clones}

a. Protocol for $\mathbf{b}$ and c. YFPCreNotch $1^{\text {flox } f \text { flox }}$ or +fflox mice were induced to give a high level of recombination and aged for 8 weeks allowing Notch1 mutant cells to colonize the epithelium. Noninduced YFPCreNotch $1^{\text {flox/flox }}$ mice were used as wild type controls $(+/+)$. Esophageal epithelium was then collected. b. Notch1 mRNA expression level measured by qRT-PCR (Mean \pm SEM, each dot represents a mouse, $n=4$ mice). One-way ANOVA; adjusted $p$ values from Tukey's multiple comparisons test against wild type. c. Immune Capillary Electrophoresis assays for NOTCH1 transmembrane/intracellular domain (NTM1 + NICD1) and $\alpha$-TUBULIN protein in esophageal epithelium (Mean $\pm S E M$, each dot represents a mouse, $n=4$ mice). One-way ANOVA; adjusted $p$ values from Tukey's multiple comparisons test against wild type. d. YFPCreNotch $1^{+/+}, Y F P C r e N o t c h 1^{+f f l o x}$ and flox/flox mice were induced at clonal density. YFP+ wild type and YFP+ Notch1 mutant clones (+/- or -/-) were analyzed at several time points (Extended data Fig. $\mathbf{2 c}$ and $\mathbf{3}$ ). e. Projected view of 4 weeks postinduction (p.i.) wild type, Notch $1^{+-}$and Notch $1^{-}$clones stained with NOTCH1 and YFP. White dashed lines delineate mutant clones. Scale bars: $30 \mu \mathrm{m}$. f. Violin plots depicting number of basal cells per clone at 4 weeks post-induction (p.i.). Lines show median and quartiles. ( $n=143$ clones from $3+/+$ mice, $n=97$ clones from $4+/$ flox mice, $n=63$ clones from 9 flox/flox mice). One-way ANOVA; adjusted $p$ values from Tukey's multiple comparisons test against $+/+$. g. Protocol for short term clonal lineage

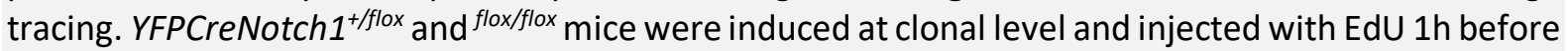
collection. h. EdU+ cells were counted within the mutant YFP+ area (green), the wild type area at the edge of the mutant clone (orange) or distant from the clone (beige). $\mathbf{i}, \mathbf{j}$. Basal EdU positive and total basal cells were counted in Notch1 ${ }^{+/}$(i) or Notch ${ }^{-/}(\mathbf{j})$ mutant clones, in wild type cells at clone edges or distant from clones. Ratio of EdU positive basal cells to total number of basal cells was calculated for each cell population (Mean \pm SEM, each dot represents a mouse; $i, n=4830 / 1584 / 4607$ cells in +/+ distant/ +/+ edge/ +/- clones from 4 mice; $j, n=3967 /$ 1036/ 4279 cells in +/+ distant/+/+ edge/ /- clones from 4 mice). One-way ANOVA; adjusted $p$ values from Tukey's multiple comparisons test against $+/+$ distant. $\mathbf{k}$. Protocol for short term clonal lineage tracing. Mice were induced at clonal level and injected with EdU 48h before collection. I. Side view of projected confocal z stacks of a Notch $1^{+-}$ clone at 3 months p.i. (left) and a Notch $1^{-}$clone at 1 month (p.i. right) from animals injected with EdU 48h before collection. NOTCH1 (magenta); YFP (green); EdU (grey); DNA (blue). Yellow dashed lines show the edges of the clones. Orange arrow points to a differentiating cell adjacent to a clone. Images are representative of clones analyzed in respectively $3 \mathrm{Notch}^{+/-}$and 5 Notch $1^{-1}$ mice. Scale bars: $30 \mu \mathrm{m} . \mathbf{m}, \mathbf{n}$. EdU was injected as in $\mathbf{k}$. EdU positive cells were counted in Notch ${ }^{+/-}(\mathbf{m})$ or Notch1 ${ }^{-/}(\mathbf{n})$ mutant clones, in wild type cells at the edge (+/+ edge) or distant from (+/+ distant) clones. Ratio of EdU positive suprabasal cells was calculated for each cell population. (Mean $\pm \mathrm{SEM}$, each dot represents a mouse; $m, n=471 / 300 / 525 \mathrm{EdU}+$ cells in +/+ distant/+/+ edge/ +/- clones from 3 mice; n, $n=1304 / 723 / 1318$ EdU+ cells in +/+ distant/ +/+ edge/ -/- clones from 5 mice). One-way ANOVA; adjusted $p$ values, Tukey's multiple comparisons test against $+/+$ distant. $A U$, arbitrary unit. 


\section{Extended Data Figure 3}

a.

YFPCreNotch1 1+/; +fflox or floxfllox

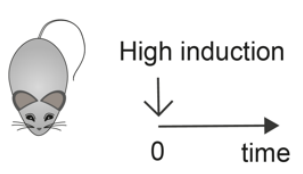

C.

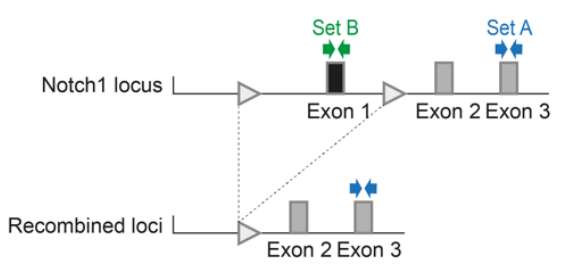

f.

YFPCreNotch 1+/+; +fflox or flloxfflox

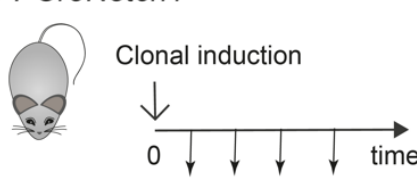

g.

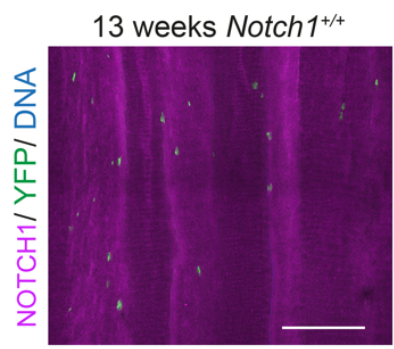

$\mathrm{h}$.

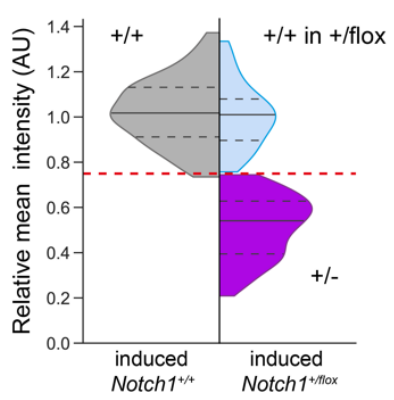

i.

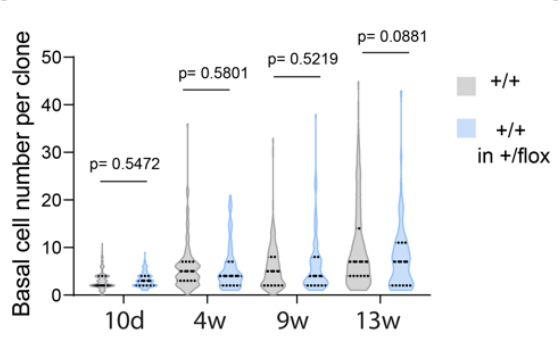

j.

k.
Notch $1^{+/+}$

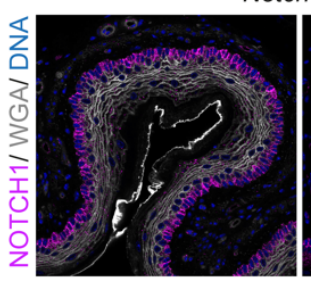

d.

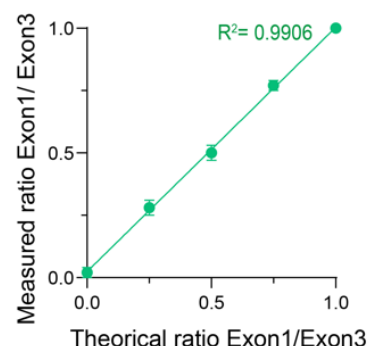

Induced Notch $1 \%$

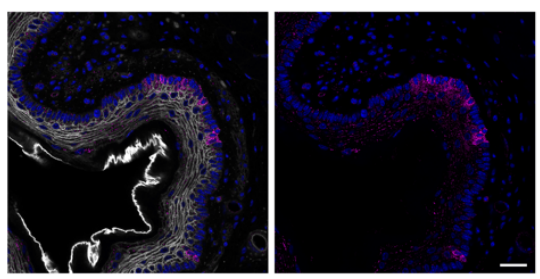

e.

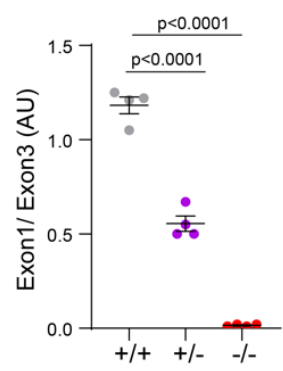

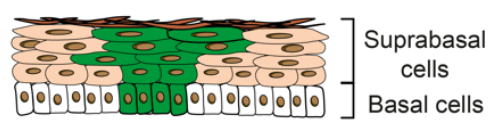

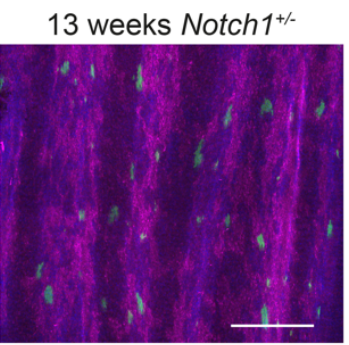

13 weeks Notch 1 -
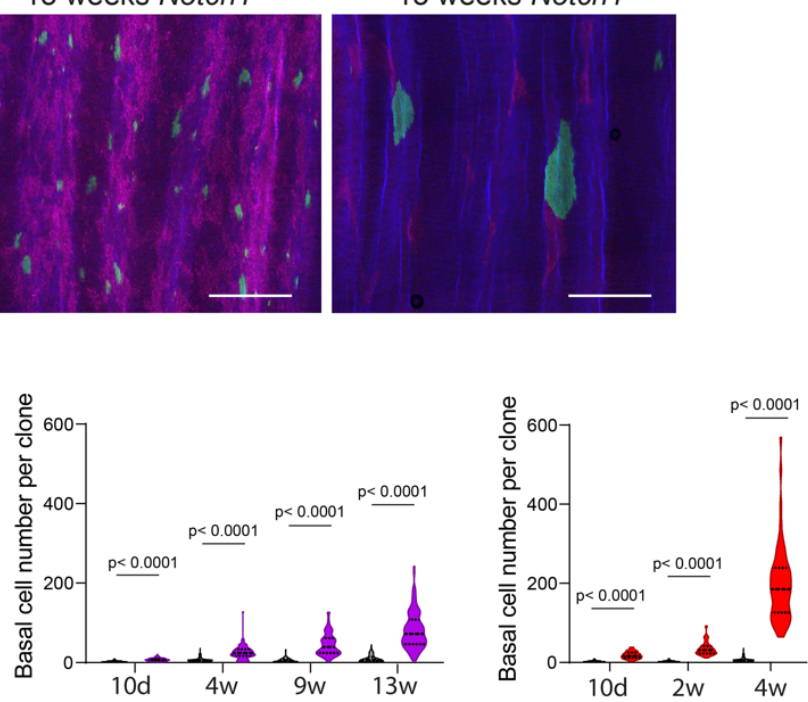

口+/+

마

- $1-$
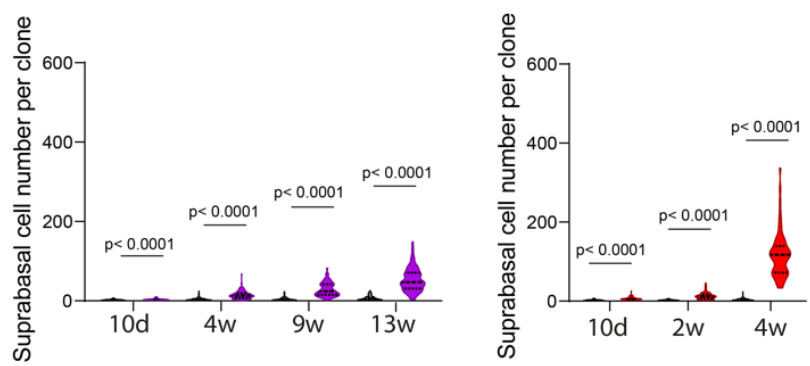


\section{Extended data Figure 3: Dynamics of Notch1 ${ }^{+/}$and Notch1\% clones}

a. Protocol for b-e. YFPCreNotch $1^{\text {flox/flox }}$ or ${ }^{+/ f l o x}$ mice were induced to give a high level of recombination and aged to allow Notch1 mutant cells to colonize the epithelium. Non-induced YFPCreNotch1 $1^{\text {floxflox }}$ mice were used as wild type controls $(+/+)$. Esophageal epithelium was then collected. b. Immunostaining of transversal section of esophageal tissue 10 days after high induction. NOTCH1 (magenta) expression is lost in most basal cells in induced Notch $1^{\text {flox/flox }}$ mice. Wheat germ agglutinin

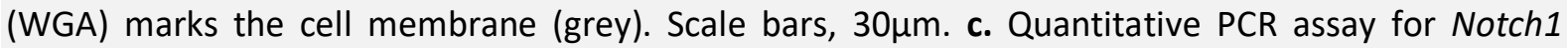
recombination. Primer set $B$ measures the loss of the floxed exon1 of Notch1 by Cre excision after induction protocol. Primer set A allows intragenic normalization within Notch1 exon 3. d. Validation of the linearity of the recombination assay against a standard curve reproducing different recombination rates with Exon1/Exon3 ratio of $1,0.75,0.5,0.25$ and $0 . \mathrm{AU}$, arbitrary units. e. Recombination assay was performed on the esophageal epithelia shown in Fig.2a-c. Exon1/Exon3 ratio is halved in induced Notch $1^{+-}$mice compared to wild type mice and null in induced Notch $1^{--}$mice suggesting full recombination of the esophageal epithelium. Mean \pm SEM, each dot represents a mouse, $n=4$. One-way ANOVA; adjusted, $p$ values from Tukey's multiple comparisons test against wild type. f. Protocol for genetic lineage tracing. YFPCreNotch $1^{\text {flox } / f l o x}$ or ${ }^{+/ f l o x}$ or ${ }^{+/+}$mice were induced at clonal level, tissue was collected, tissue was peeled and stained by whole mount for NOTCH1, YFP and DAPI and cohorts of YFP+ clones imaged at different time points. Basal and suprabasal cells were counted in mutant or control clones categorized on the basis of NOTCH1 staining (Extended data Fig.2a). g. Notch $1^{+/+}$, Notch $1^{+/-}$and Notch $1^{-/}$clones 13 weeks post clonal level induction in esophageal epithelial wholemounts stained for NOTCH1 (magenta), YFP (green) and DNA (blue). Scale bars, $500 \mu \mathrm{m}$. h. d. YFPCreNotch $1^{+/+}$and YFPCreNotch $1^{+/ f l o x}$ mice were induced at clonal level and intensity of NOTCH1 staining was measured in the basal layer of YFP+ clones. Recombination of YFP is independent of the Notch1 locus so YFP clones may be Notch $1^{+/+}$or Notch1 $1^{+-}$in YFPCreNotch1 $1^{+/ f l o x}$ animals depending on whether Notch1 was recombined. Intensity of clones in YFPCreNotch $1^{+/+}$mice served as reference for discriminating Notch1 wild type from Notch $1^{+-}$clones in the induced Notch $1^{+/ f l o x}$ mice. Violin plot shows the distributions of relative NOTCH1 staining intensity of YFP+ clones in $\mathrm{Notch}^{+/+}$mice (left, grey, $\mathrm{n}=3$ mice) and Notch $1^{+/-}$mice (right, $\mathrm{n}=7$ mice). Black lines show median and quartiles. Red dashed line indicates the threshold relative intensity (75) determined based on the normal approximation of the distribution in wild type mice and the two-component Gaussian mixture model approximation of the distribution in induced Notch $1^{+/ f l o x}$ mice. Clones with intensity $>75$ in induced Notch $1^{+/ f l o x}$ mice are categorized as wild type (+/+ in $+/$ flox, blue), the other clones are categorized as $\mathrm{Notch}^{+/-}(+/-$, purple). i. Validation of clonal genotype from NOTCH1 immunostaining. Violin plots show the number of basal cells/clone in wild type $(+/+)$ and wild-type intensity populations from induced Notch $1^{+/}$tissue (+/+ in $+/$flox). Lines show median and quartiles. Groups do not show statistical difference in clonal size at 10 days, 4,9 and 13 weeks. Number of mice (clones) for $+/+$ and +/+ in +/flox respectively at 10 days: 3 (206)/ 5 (94); 4 weeks: 3 (143)/ 4 (78); 9 weeks: 3 (132)/ 4 (72); 13 weeks: 3 (126)/ 7 (65). Two tailed unpaired Student's t-test against +/+ at each time point. j, k. Basal cell number $(\mathbf{j})$ and suprabasal cell number $(\mathbf{k})$ per clone is shown with violin plots at different times after induction. YFP positive Notch1 ${ }^{+/}$(left panel) or Notch1 ${ }^{-/}$(right panel) clonal cell counts are shown in comparison to Notch $1^{+/+}$clones. Lines show median and quartiles. Fusion of expanding clones prevented quantification of clone size beyond 4 weeks for Notch $1^{f l o x}$ flox tissue and beyond 13 weeks for Notch $1^{+/ f l o x}$ tissue. Number of mice (clones) for $+/+$ at 10 days, 2 weeks, 4 weeks, 9 weeks, 13 weeks respectively: 3 (206)/ 3 (155)/ 3 (143)/ 3 (132)/ 3 (126). Number of mice (clones) for +/- at 10 days, 4 weeks, 9 weeks, 13 weeks respectively: 5 (84)/ 4 (97)/ 4 (68)/ 7 (107). Number of mice (clones) for -/at 10 days, 2 weeks, 4 weeks respectively: $6(68) / 3(69) / 9(63)$. P values from two-tailed unpaired Student's t-tests against $+/+$ at each time point. 
bioRxiv preprint doi: https://doi.org/10.1101/2021.06.18.448956; this version posted June 18, 2021. The copyright holder for this preprint (which was not certified by peer review) is the author/funder, who has granted bioRxiv a license to display the preprint in perpetuity. It is made available under aCC-BY 4.0 International license.

These observations and constraints were integrated into a simple Wright-Fisher style quantitative model of 'neighbor constraint', in which competition is driven by local fitness differences between cells. Mutant clones expand until they collide with other mutant clones of similar fitness, at which point they revert to neutral competition ${ }^{19}$. We fitted this model to the clone size data to estimate the fitness of mutant clones relative to wild type cells with a fitness of 1 . The inferred fitness for Notch $1^{+/-}$ clones was substantially greater than that of wild type cells and the inferred fitness of Notch $1^{-}$clones markedly higher than that of heterozygous clones (Extended data Fig. 4a-d, Video 1, Supplementary Note). We conclude that loss of one allele of Notch1 is sufficient to drive clonal expansion, but the competitive fitness of clones is substantially augmented when the second allele is lost.

\section{Extended Data Figure 4}

a.

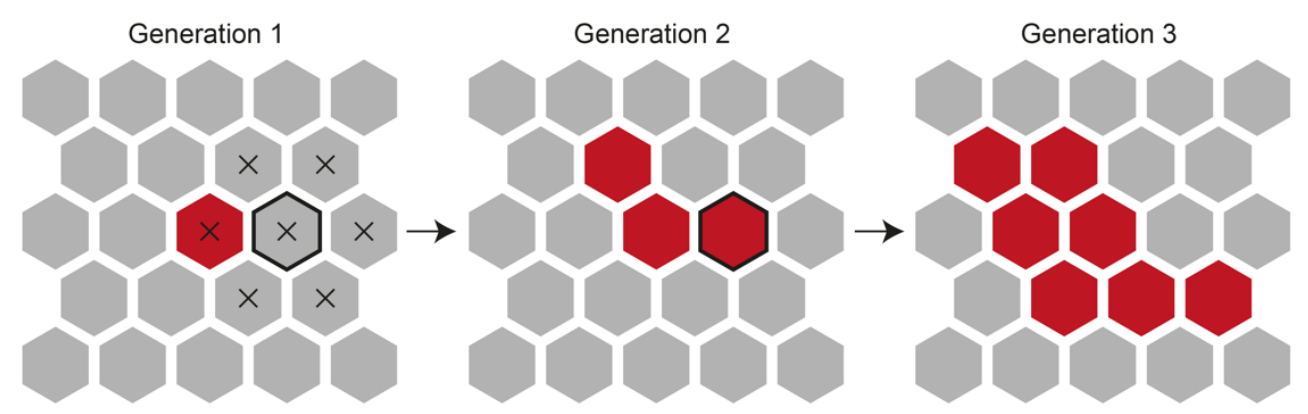

b.

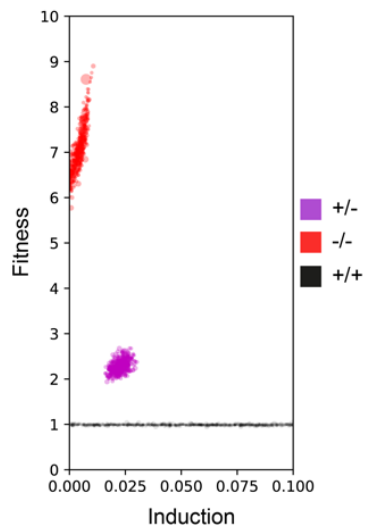

e.

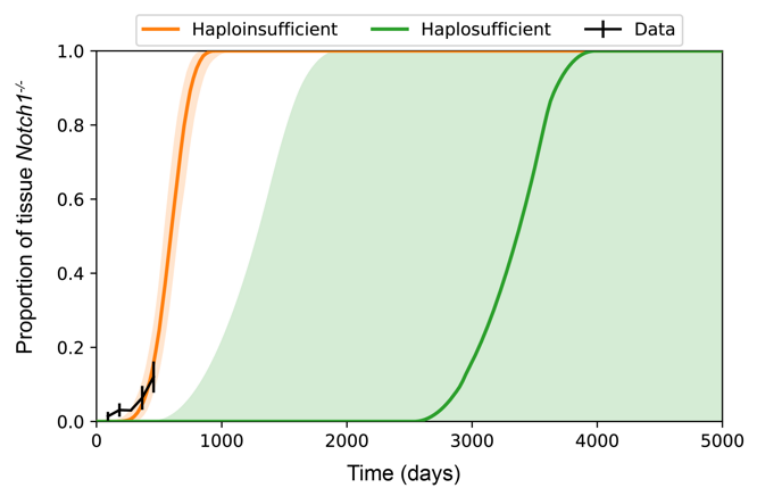

C.

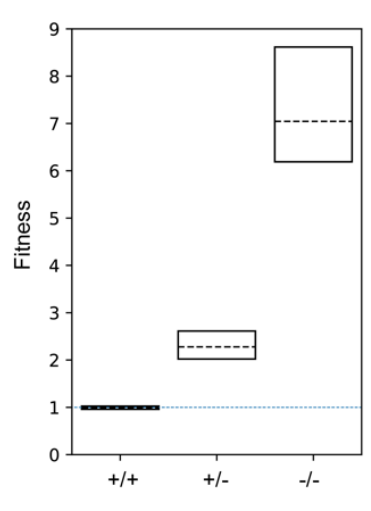

d.

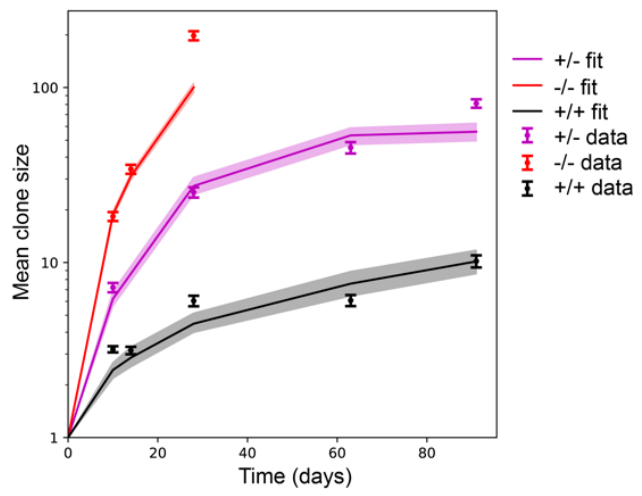

f.

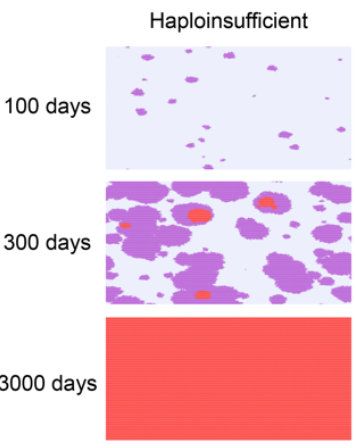

Haplosufficient 
bioRxiv preprint doi: https://doi.org/10.1101/2021.06.18.448956; this version posted June 18,2021 . The copyright holder for this preprint (which was not certified by peer review) is the author/funder, who has granted bioRxiv a license to display the preprint in perpetuity. It is made available under aCC-BY 4.0 International license.

\section{Extended Data Figure 4: Modelling Notch1 mutant clone expansion}

a. 2-dimensional Wright-Fisher style model of clone dynamics in the esophagus. The basal layer is made up of a hexagonal grid of cells. At time zero, a small proportion of cells are assigned to be mutant (red) and the rest wild type (grey). Cells in the next generation are picked from neighboring cells - e.g. the cells which can be placed in the outlined position in generation 2 are those marked with an X in generation 1. Mutant cells with higher fitness have a higher probability of generating daughter cells in the next generation and hence can expand into large clones (Supplementary Note). b. Plot shows the inferred induction proportion and inferred fitness values from $A B C$ fitting to the lineage tracing data (Supplementary Note) for Notch1\% (red), Notch1 ${ }^{+/-}$(purple) and Notch1 $1^{+/+}$(black) animals. Each dot shows an "accepted" parameter set. c. Distributions of acceptable values of the fitness parameter. Dashed lines and box show median inferred fitness and 95\% credible intervals. A fitness of 1 is neutral. d. Mean clone sizes from simulations of the parameters at the peak of the acceptable distributions (see Supplementary note). Median and 95\% confidence intervals of 100 simulations shown for the simulation curves. Mean \pm SEM are shown for the experimental data. e. Proportion of tissue covered by Notch $1 \%$ clones over time in simulations using the best fit fitness parameter for Notch $1 \%$ fitness. Notch $1^{+/}$is either assumed to be neutral (haplosufficient, green) or to have the best fitting fitness parameter to the experimental analysis of $\mathrm{Notch}^{+/-}$clones (haploinsufficient, orange). Curves show median and shaded areas show $95 \%$ confidence intervals. f. Snapshot images at 100 days, 300 days and 3000 days of video modelling using the simulations shown in e. On the left, Notch ${ }^{+/-}$cells are haploinsufficient (fitting to observed experimental data), on the right the Notch1 ${ }^{\text {+/ }}$ cells are assumed to be haplosufficient (neutral fitness). See Supplementary Note. 


\section{Notch1 haploinsufficiency is pivotal in the colonization of normal epithelium}

Clones generated by the transgenic deletion of Notch1 alleles may not reflect the behavior of the sporadic Notch1 mutant clones carrying truncating, missense or indel mutations that develop during aging. We therefore investigated the appearance of spontaneous Notch1 mutant clones in control YFPCreNotch $1^{+/+}$mice, comparing this with highly induced YFPCreNotch $1^{+/}$animals which we used to model the effects of loss of a second Notch1 allele within heterozygous epithelium. Both strains were aged for over a year prior to immunostaining the epithelium for NOTCH1 (Fig. 3a). We observed a progressive increase in the area of epithelium staining negative for NOTCH1 protein in both induced strains, rising to $12 \%$ of Notch $1^{+/+}$and $78 \%$ of Notch ${ }^{+/}$epithelium by 65 weeks (Fig. 3b,c). Widespread loss of NICD1 staining was also observed in the aged Notch1 $1^{+-}$tissue (Extended data Fig. $\mathbf{5 a}, \mathbf{b})$. These findings show that esophageal epithelium is colonized by Notch1 mutant clones during aging, as is the case in humans, and that loss of the remaining Notch1 allele in Notch $1^{+/-}$epithelium increases competitive fitness.

In order to isolate and sequence potential clones we used NOTCH1 staining in combination with the YFP reporter. Aging Notch1 ${ }^{+/}$epithelium contained multiple ovoid areas far larger than most YFP labelled clones. Some of these were positive for YFP (YFP+), whereas in others all YFP clones were absent (YFP-), suggesting they had been displaced by an expanding clone (Fig. 3d,e). These NOTCH1 positive and negative 'expanded' areas were imaged, dissected out and targeted sequencing of 73 Notch pathway and cancer related genes was performed on a total of 246 biopsies (Supplementary tables 1 and 4). We analyzed CNLOH and all mutations with a VAF higher than 0.2 , as below this threshold mutations were considered unlikely to drive the presumed clonal expansion. 97\% (180/185) of the sequenced YFP+ and YFP- expanded areas had either Notch1 protein-altering mutations with VAF $\geq 0.2$ or $\mathrm{CNLOH}$ at Chromosome 2 involving the Notch1 locus (GRCm38 - chr2:26,457,903$26,503,822)$. In contrast, only $2 / 61$ samples from non-expanded areas carried Notch1 mutations and none had CNLOH (Fig. 3f, Extended data Fig. 5c, d, Supplementary table 4). A small number of mutations in other genes were also called with $\mathrm{VAF} \geq 0.2$, however they were either found coupled with a high VAF Notch1 genetic alteration, so may represent passenger mutations within a Notch1 mutant clone or were found in non-expanded areas. Expanded areas were thus associated with genetic events affecting Notch1 locus. Furthermore, 94\% (169/180) of the expanded areas with Notch1 altering events carried only a single event (about $50 \%$ one Notch1 protein-altering mutation and the remainder $\mathrm{CNLOH}$ ) with an average VAF 0.44 , consistent with them being clones carrying spontaneous genetic events affecting the non-recombined Notch1 allele. (Fig. 3e-g, Supplementary Table 4, Extended data Fig. 5c,d). NOTCH1 expressing clones harbored missense mutations (87\%) or Indels (13\%) (Fig. 3g). Overall the effect of these mutations on NOTCH1 expression is consistent with our observations in human tissue (Fig.1).

To test the functional impact of missense mutations we used an ex-vivo assay of NOTCH1 function Extended data Fig. 5e-j, Supplementary table 4) ${ }^{20}$. YFPCreNotch $1^{+/-}$tissues analyzed in Fig. 3e-g were incubated with EDTA at $37^{\circ} \mathrm{C}$ prior to fixation. Depletion of Ca2+ using EDTA promotes the cleavage of NEC and NTM subunits without ligand binding, thought to be due to conformational changes in the LNR domains (Extended data Fig.1a) ${ }^{20}$. This results in the release and nuclear migration of NICD. Some NOTCH1 positive clones displayed NICD nuclear staining, but in others nuclear NICD was absent (Extended data Fig, 5e). Nuclear NICD staining clones were enriched in missense mutations located in the ligand binding site, EGF repeats 8-12, whereas non-nuclear staining clones were enriched in NRR domain mutants (Extended data Fig. 5g, h p=0.001, Chi-square test). Most of the mutated residues 
bioRxiv preprint doi: https://doi.org/10.1101/2021.06.18.448956; this version posted June 18, 2021. The copyright holder for this preprint (which was not certified by peer review) is the author/funder, who has granted bioRxiv a license to display the preprint in perpetuity. It is made available under aCC-BY 4.0 International license.

in the ligand binding domain had highly destabilizing properties, consistent with them impairing Notch signaling by disrupting ligand binding, a process bypassed in the ETDA assay (Extended data Fig. 5i) ${ }^{21,22}$. The NRR domain mutants were clustered in the LNR1 and 2 domains (Extended data Fig. 5j) ${ }^{23}$. This contrasts with NOTCH1 activating mutations in human T cell acute lymphoblastic leukemia (TALL), collated from COSMIC (https://cancer.sanger.ac.uk/cosmic), which cluster in the HD domain of the NRR and promote the cleavage of NEC without ligand interaction (two-sided Fisher exact test comparing mutation counts in the LNR1-2 and LNR3-HD sub-regions of the NRR, $p=1.48 \mathrm{e}^{-10}$ Extended data Fig. 5j) ${ }^{24,25}$. This observation associated with the absence of detectable nuclear NOTCH1 after EDTA treatment in this group of clones suggests that the mutations they carry at the NRR domain may lock it into its auto-inhibited conformation, preventing the cleavage of NOTCH1.

\section{Figure 3}

a.

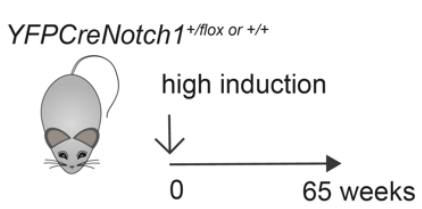

d.

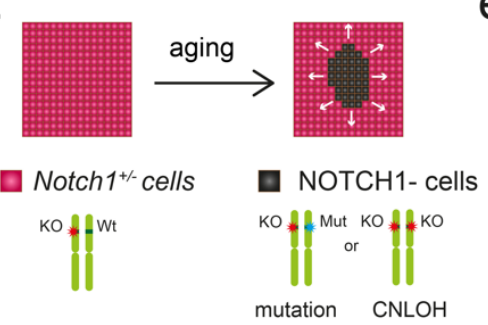

f.

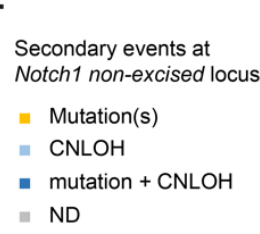

g.
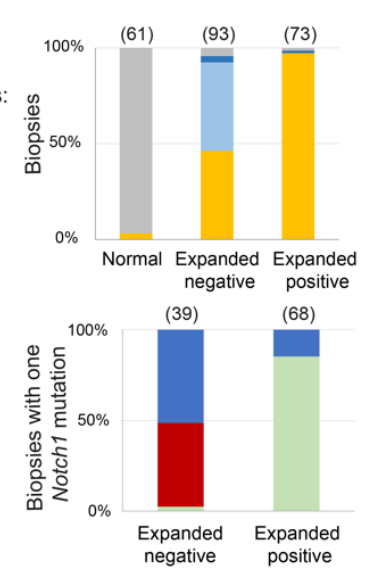

b.

e.

Missense

- Nonsense

- Indel/splicing
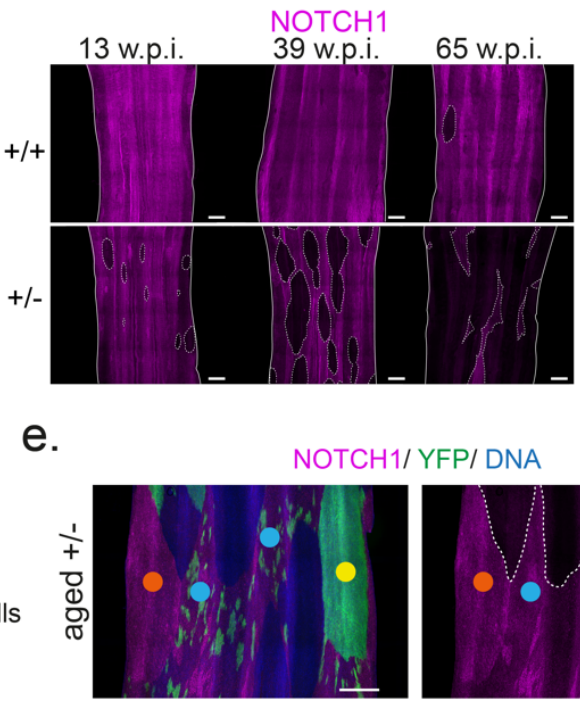

h.

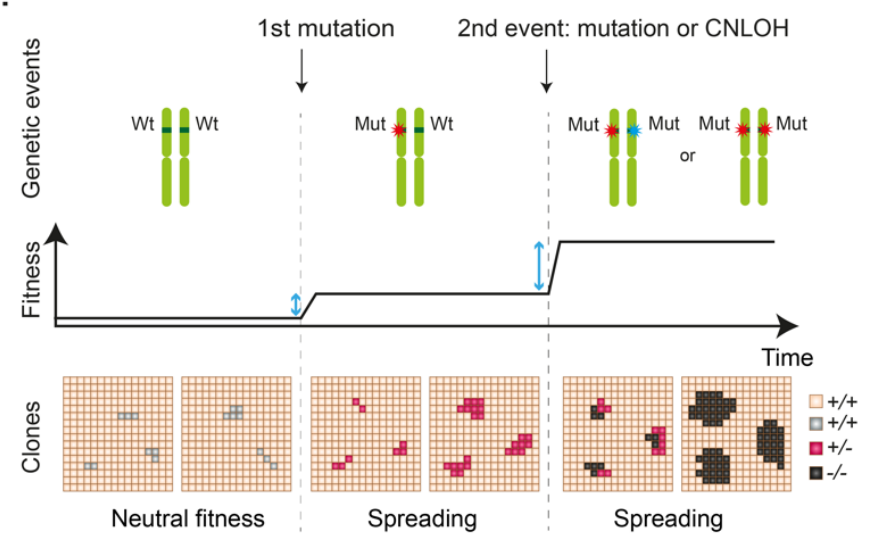

C.

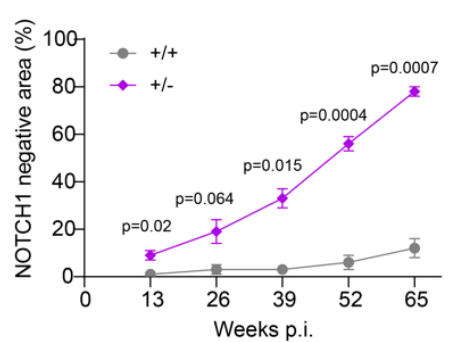

Sampled areas

- Non expanded normal area

- Expanded negative area

- Expanded positive area 
bioRxiv preprint doi: https://doi.org/10.1101/2021.06.18.448956; this version posted June 18, 2021. The copyright holder for this preprint (which was not certified by peer review) is the author/funder, who has granted bioRxiv a license to display the preprint in perpetuity. It is made available under aCC-BY 4.0 International license.

\section{Figure 3: Notch1 colonizes aging esophageal epithelium}

a. YFPCreNotch $1^{+/ f l o x}$ and ${ }^{+/+}$mice were induced at high level and aged for 65 weeks. b. Representative NOTCH1 staining in esophageal epithelium of Notch $1^{+/+}$and $\mathrm{Notch}^{+/-}$mice at the indicated time points. White dashed lines delineate negative areas, solid lines delineate tissue edges. Images are representative confocal $z$ stack of entire epithelium from 3 mice per time point. W.p.i, weeks- postinduction. c. Percentage of NOTCH1 negative area increases with age in Notch $1^{+/+}$(Kendall's tau-b correlation $=0.56, p=0.0062$ ) and Notch $1^{+-}$(Kendall's tau-b correlation=0.91, $p=8.3 e-6$ ) esophagi (Mean \pm SEM, $n=3$ mice per time point). $P$ values shown are from two-sided Welch's t-test. Scale bars: $500 \mu \mathrm{m}$. d. Schematic of Notch1 $1^{+-}$cells (purple cells) showing the spontaneous appearance of expanding NOTCH1 negative cells (black) with aging, possibly caused by genetic events affecting the Notch1 locus. e. Induced YFPCreNotch $1^{+-}$mice were aged 54 to 78 weeks-old, when esophageal epithelium was collected and stained for NOTCH1 (magenta) and YFP (green). Expanding areas devoid or fully stained with YFP appeared distinct from normal appearing areas marked with a patchwork of small YFP+ clones. Expanded NOTCH1 negative (yellow) and positive (orange) areas and normal appearing areas (blue) were isolated for targeted sequencing ( $n=246$ biopsies from 10 mice). Colored circles show the sampled areas. White dashed lines delineate negative areas. Scale bar: $500 \mu \mathrm{m} . \mathrm{f}$. Proportion of normal appearing, expanded NOTCH1 negative and expanded NOTCH1 positive biopsies with Notch1 mutations or copy number alternations. g. Proportion of NOTCH1 negative and positive areas carrying a secondary missense, nonsense or indel/splicing Notch 1 mutation. For $\mathbf{f}$ and $\mathbf{g}$, number of samples is shown in brackets, redundant samples, defined as biopsies sharing the same mutation and separated by $<1 \mathrm{~mm}$ were counted once. $\boldsymbol{h}$. Summary scheme of colonization by Notch1 clones. Clonal fitness increases from monoallelic and biallelic Notch1 mutation resulting in a selective pressure (blue arrows) for biallelic gene alterations. P.i., post-induction, Wt, wild-type; KO, knock-out allele lacking Notch1 exon 1; Mut, mutation., ND, none detected. See Supplementary table 4. 


\section{Extended Data Figure 5}

a.

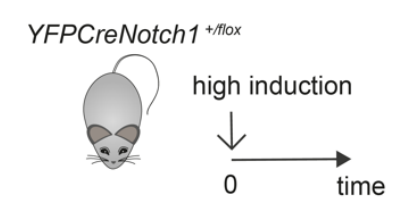

C.

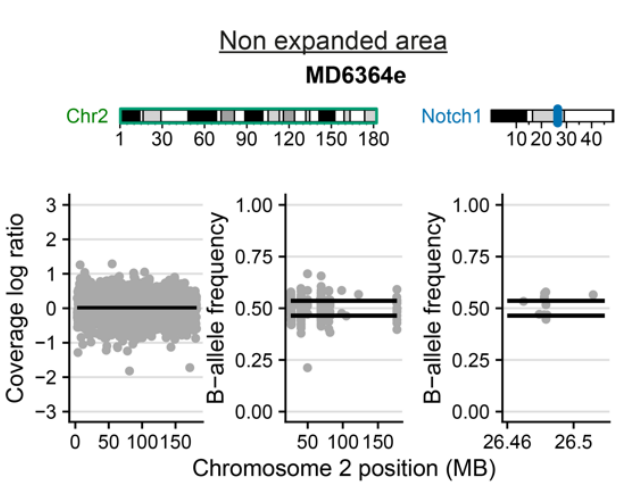

b.

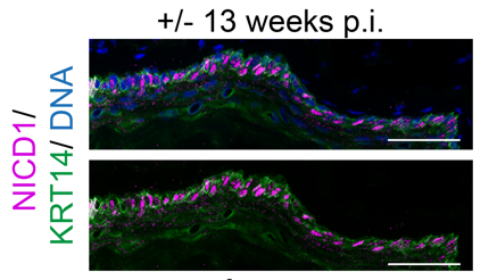

d.

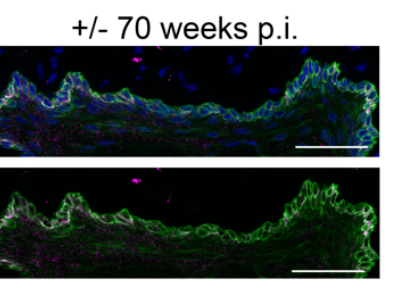

NOTCH1 negative expanded area MD6364j
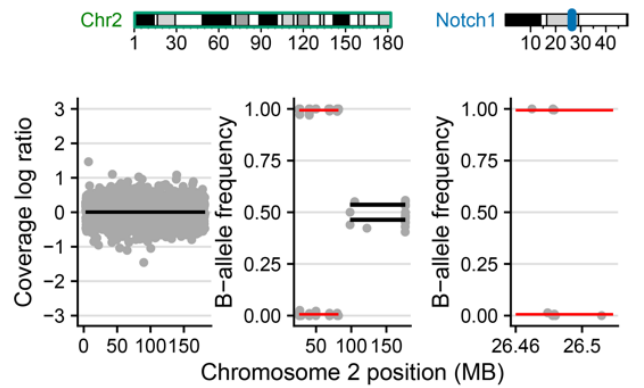

e.
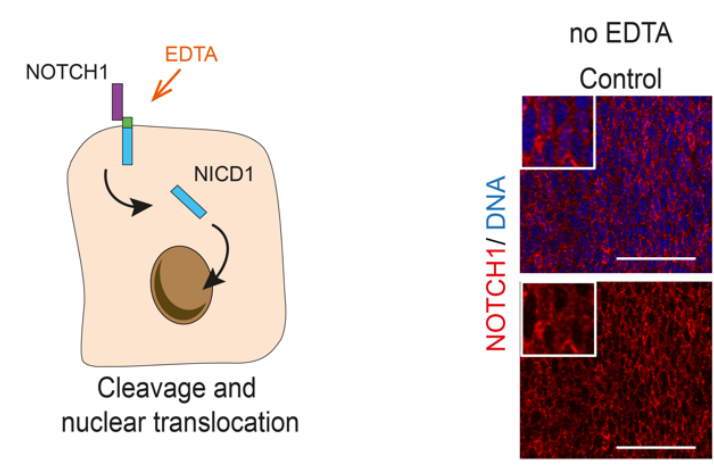

f.

g.

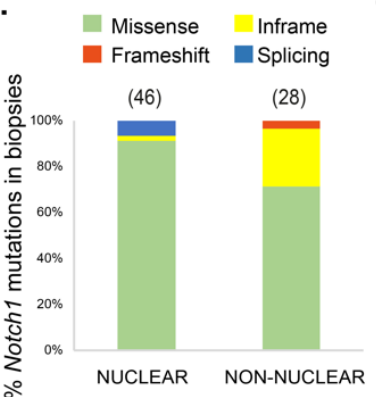

i.

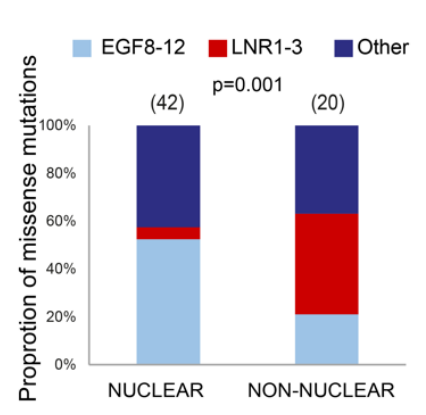

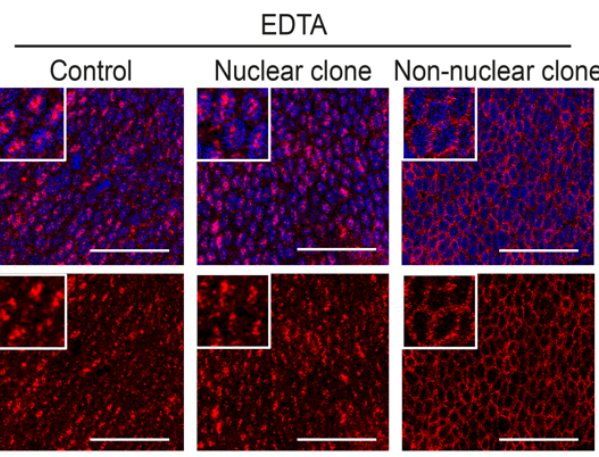

h.

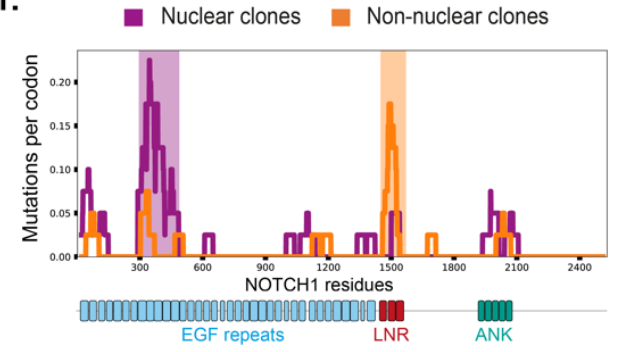

NOTCH1 EGF 8-12

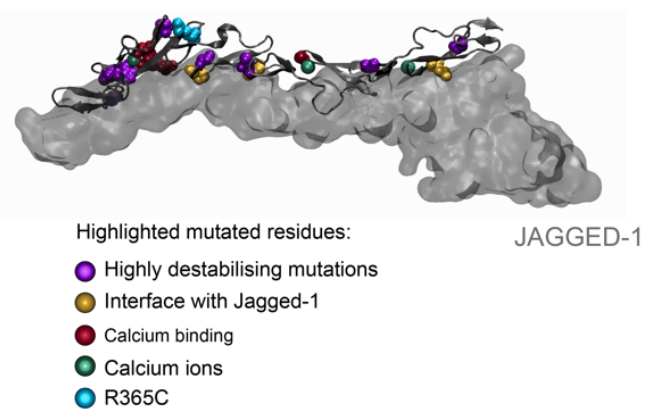

j.

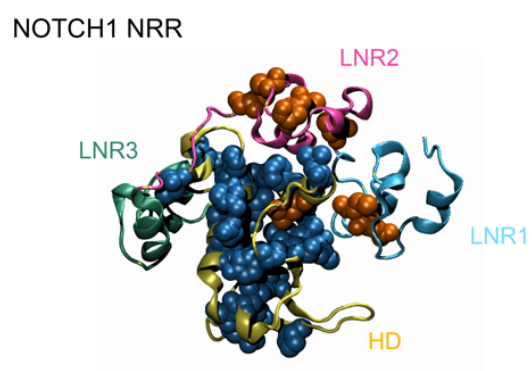

Mutated residues from non-nuclear biopsies

- Mutated residues in TALL samples (COSMIC) 
Extended Data figure 5: Analysis of spontaneous mutant clones in Notch1 ${ }^{+/}$aged esophageal epithelium

a. Protocol. YFPCreNotch1 $1^{+/ f l o x}$ mice were induced at high density and aged up to 78 week-old. b. Representative NICD1 (magenta) and KRT14 (green) staining of sections of esophagi from Notch1 ${ }^{+/}$ mice, 13 weeks and 70 weeks after induction. Images are representative examples from 3 and 5 mice respectively. Scale bars, 50 um. c, d. As in Fig. 3e, expanded and non-expanded areas were dissected and targeted sequencing performed. Plots show representative examples of copy neutral LOH analysis from non-expanded normal area (c) and NOTCH1 negative expanded area carrying CNLOH (d) Total copy number and loss of heterozygosity analysis from targeted sequencing. The left panel contains the read coverage of off-target reads across chromosome 2 and shows no changes in total copy number were detected. The middle and right panels show the B allele fraction (BAF) of germline heterozygous SNPs along chromosome 2 (middle) and at the Notch1 locus (right). Significantly different values for total copy number or BAF imbalance are shown with red lines. The NOTCH1 negative sample MD6364j shows CNLOH at clonal level. e. Left panel. Aged YFPCreNotch ${ }^{+/-}$mice esophagi were incubated in $5 \mathrm{mM}$ EDTA solution, activating NOTCH1 cleavage in the absence of ligand, stained for NOTCH1 (red), YFP and DNA (blue) and control and clonal areas were sampled and identified as shown in Fig. 3e-g. Right panel shows representative pictures of NOTCH1 positive areas. Non expanded normal areas show nuclear NOTCH1 only following EDTA treatment as opposed to nonEDTA treated cells displaying both nuclear and non-nuclear staining. EDTA treated positive clones display either nuclear staining (nuclear clone) or extra nuclear NOTCH1 (non-nuclear clone). Scale bars, $50 \mu \mathrm{m}$. Analysis of the type of Notch 1 mutations in mutant clones with NOTCH1 nuclear staining (nuclear) and without nuclear staining (non-nuclear) imaged as in e. Number of samples is indicated in brackets. Redundant samples defined as biopsies sharing the same mutation and spaced $<1 \mathrm{~mm}$ away in the tissue were counted once. g. Location of missense mutations in nuclear and non-nuclear samples in NOTCH1 protein. Number of samples is indicated in brackets. $\mathrm{P}=0.001$, Chi-square test.h. Distribution of NOTCH1 codon alterations in missense mutants in nuclear (purple, $n=42$ mutations) and non-nuclear (orange, $n=20$ ) clones. Purple shadow highlight the cluster of mutations in EGF repeat $8-12$, Orange shadow highlight the cluster of mutations at the LNR repeats. i. Missense mutations in NOTCH1 EGF8-12 in samples with nuclear staining. Residues containing missense mutations are highlighted on the structure of rat Notch1 EGF8-12 bound to Jagged-1 (PDB 5UK5). Mutated residues involved in calcium binding are shown in dark red, residues on the interface with NOTCH1 ligands are shown in yellow, and residues with highly destabilizing mutations (FoldX $\Delta \Delta \mathrm{G}>2 \mathrm{kcal} / \mathrm{mol}$ ) are shown in purple. R365C, which does not fit into any of the previous categories is shown in blue. Calcium ions are shown in green, $n=21$ missense mutations. $j$. Mutated residues highlighted on the 3D human NRR structure (PDB 3ETO). Missense mutations in NOTCH1 NRR in biopsies with non-nuclear staining are shown in orange ( $n=9$ mutations). Activating missense mutations from human T-cell acute lymphoblastic leukemia (T-ALL) samples from COSMIC (https://cancer.sanger.ac.uk/cosmic ${ }^{25}$ ) are shown in blue ( $n=153$ mutations). The proportion of missense mutations between LNR1-2 and LNR3HD in T-ALL and non-nuclear NOTCH1 stained esophagus is significantly different (Two-sided Fisher exact tests, $p=1.48 \mathrm{e}^{-10}$ ). LNR1, blue; LNR2, pink; LNR3, green; HD domains, yellow. See Supplementary table 4.

We conclude that most NOTCH1 expressing clones carry mutations disrupting NOTCH1 function. Overall, the emergence of large clones carrying mutations or CNLOH in Notch1 heterozygous mice is consistent with spontaneous mutation of the remaining Notch1 allele conferring a fitness advantage over neighboring heterozygous cells.

Collectively these observations reveal that haploinsufficiency is key for the normal esophagus to be colonized so effectively by Notch1 mutants. In simulations, neutral mutations do not spread across the tissue and are likely to disappear from the tissue over time. However, loss of one allele biases 
mutant progenitor cell fate towards the production of progenitors, increasing the likelihood that mutant clones will expand and persist in the epithelium (Extended data Fig. 4e, f, Video 2) Notch1 inactivated cells have a further fitness advantage, so that subclonal loss of the second allele within a persisting heterozygous clone will generate cells that outcompete both $\mathrm{Notch}^{+/+}$and $\mathrm{Notch}^{+/-}$ neighbors (Fig. 3h). Over time biallelic clones will thus populate the tissue. This model explains the high prevalence of clones with NOTCH1 mutation and $\mathrm{CNLOH}$ in aging human esophagus.

\section{Notch1 loss alters transcription but not tissue function}

The replacement of large areas of the esophageal epithelium by clones lacking functional NOTCH1 might be expected to have cellular and tissue level phenotypes. To explore the effects of Notch1 loss we first performed bulk RNA sequencing on peeled esophageal epithelium from Notch1 wild type, heterozygous and homozygous mice (Fig. 4a-e). YFPCreNotch $1^{+/ f l o x}$ and YFPCreNotch $1^{\text {flox/flox }}$ mice were induced at a high level and aged for 8 weeks so that the recombined cells occupied almost all the esophageal epithelium (Fig. 4a, Extended data Fig.3e, Fig.2-c). RNA-seq was performed on the peeled esophageal epithelium from these mice and wild type littermates. In comparison with wild type tissue, 20 genes in Notch $^{+/-}$and 227 genes Notch1\%- esophagus were differentially expressed (Fig. 4c-e, Supplementary table 5). These included previously identified Notch1 regulated genes, lgfbp 3 and Sox9, but transcription of canonical Notch targets such as Hes1 and Nrarp was not significantly altered (Supplementary table 5) ${ }^{16,26,27}$. Gene Ontology analysis showed genes regulating biological processes related to squamous epithelia were not significantly affected (EnrichGO q-value $>0.05$ ), though transcripts of genes involved in DNA replication were downregulated $\left(q=2.6 \times 10^{-7}\right)$ (Supplementary table 5).

To define the effect of Notch1 loss on cell lineages and cellular states we performed single cell RNA sequencing (scRNA-seq). YFPCreNotch $1^{\text {flox } / \text { flox }}$ mice were induced at high density and aged so that the recombined keratinocytes covered the tissue entirely (Fig. 4f, Extended data Fig.6a). scRNA-seq was performed on dissociated epithelium from these mice and uninduced littermate controls (Fig. 4f-j, Extended data Fig. 6b-e, Supplementary table 6). After filtering out poor quality cells, a total of 13111 cells remained for analysis, from two biological replicates per genotype (Fig. 4g, Supplementary Note). We analyzed the cells using the Seurat software package, which identified cell clusters corresponding to four lineages (Fig. 4h, Extended data Fig. 6 c,e) ${ }^{28}$. The proportions of keratinocytes, fibroblasts immune and endothelial cells were similar in the control and Notch $1^{\%}$ esophagus (Fig. 4h). We next applied the Seurat pipeline to the keratinocytes alone (Extended data Fig. 6d). The analysis revealed the existence of a continuum of keratinocytes states, from progenitors expressing $K r 14$, to differentiating cells expressing Krt4 or Tgm3 to terminally differentiated cornified cells expressing Lor (Extended data Fig. 6e). We used the expression level of these markers to discriminate basal and suprabasal cells on the UMAP plot (Supplementary Note). The analysis revealed similar proportions of keratinocytes in each differentiation state in the control and Notch $1^{\%}$ tissue (Fig. 4i). Cell cycle gene expression patterns in keratinocytes also showed minimal differences between Notch ${ }^{1-}$ and wild type tissues (Fig. 4 j). Overall, unbiased exploratory RNA-seq approaches did not evidence major alterations in tissue function or cellular composition due to loss of Notch1 in esophageal epithelium. This conclusion was supported by imaging of the esophagus from induced and aged Notch $1^{\text {flox/flox }}$ mice and control littermates. Tissue thickness, basal cell density and immunostaining for differentiation markers KRT14, KRT4 and LOR and the proliferation marker Ki67 were similar in both genotypes, (Fig.5a-d). 


\section{Figure 4}

a.

YFPCreNotch 1 thox YFPCreNotch $1^{\text {floxflo }}$

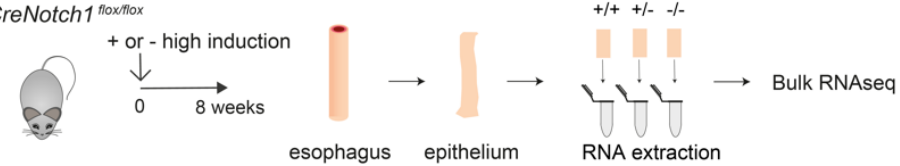

c.

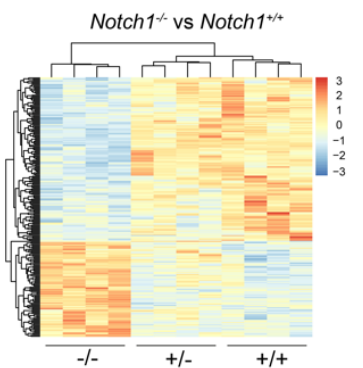

f.

YFPCreNotch 1 floxfliox
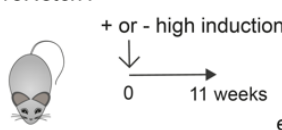

d.
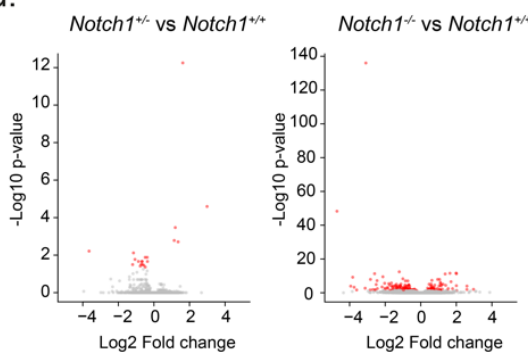

Log2 Fold change

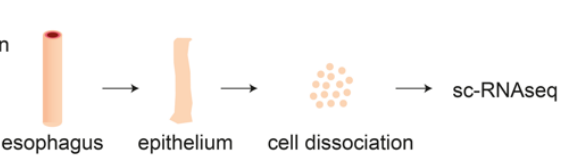

h.
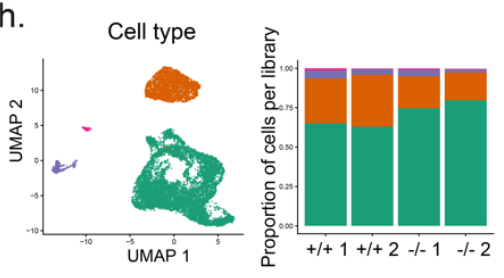

- Keratinocyte $\bullet$ Fibroblast $\bullet$ Immune $\bullet$ Endothelial $\bullet N A$
- Basal - Suprabasal

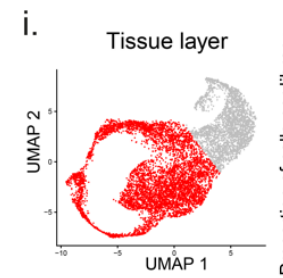

b.

e.
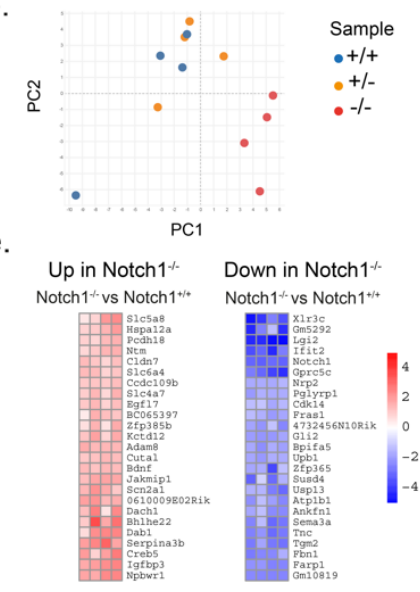

g.
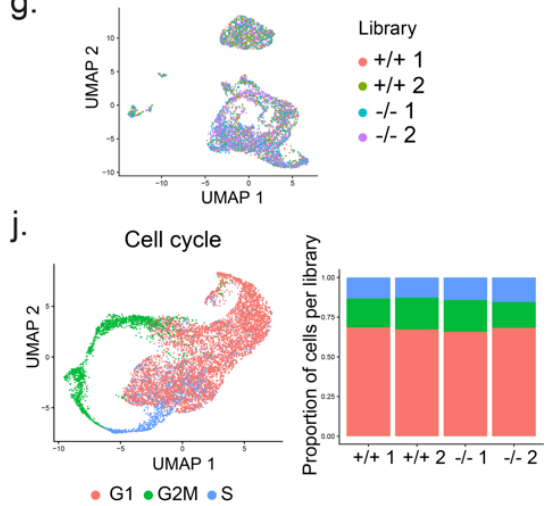

\section{Figure 4: Notch1 loss alters transcription but not tissue composition or cell dynamics}

a. YFPCreNotch $1^{\text {flox } f \text { flox }}$, YFPCreNotch $1^{\text {tfflox }}$ mice were highly induced and aged for 8 weeks to allow the mutant cells to cover the esophageal epithelium. Uninduced YFPCreNotch $1^{\text {flox } / \text { flox }}$ mice were used as wild type controls $(+/+)$. Esophageal epithelium was collected and peeled and samples were processed for bulk RNAseq ( $n=4$ mice per group). b. Principal component analysis (PCA) plot showing the four

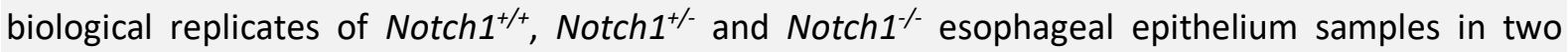
dimensions. c. Hierarchical clustering and heat map showing differentially expressed genes between Notch $1^{--}$and control tissues, in all three genotypes. d. Volcano plots showing Notch $1^{+-}$vs control (left) and Notch $1 \%$ vs control (right) RNAseq analyses. Differentially expressed genes are indicated in red e. RNA-seq generated heat maps showing Log 2 fold changes of the 25 top differentially expressed genes (DEG) in Notch1 ${ }^{-/}$compared to Notch $1^{+/+}$tissues, Dseq2 analysis, adjusted $p$-value $<0.05$. f. YFPCreNotch $1^{\text {flox/flox }}$ mice were highly induced and aged for 11 weeks, allowing the mutant cells occupy the esophageal epithelium. Controls were uninduced YFPCreNotch $1^{\text {flox/flox }}$ mice $(+/+)$. Esophageal epithelium was dissociated and sequenced. g. UMAP plot shows an overlay of 1500 cells from each library ( $n=2$ mice per genotype; $+/+1, n=2454 ;+/+2, n=3194 ;-/-1, n=1929 ;-/-2, n=5534)$. $h$. Left, UMAP plot showing cell types identified via sc-RNAseq. Right, stacked bar chart shows the proportion of cell types per library. NA, not available i. Left, UMAP plot of keratinocytes. Right, stacked bar chart shows the estimated proportion of keratinocytes per library belonging to the basal or suprabasal layers. $\mathbf{j}$. Left, UMAP plot of keratinocytes and the likely cell cycle phase they belong to (G1, G2/M or S). Right, stacked bar chart showing proportion of keratinocytes per library assigned to each cell cycle phase. See Supplementary tables $\mathbf{5}$ and $\mathbf{6}$. 


\section{Extended data Figure 6}

a.

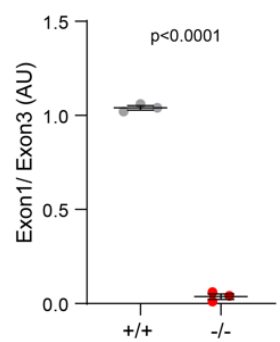

d.

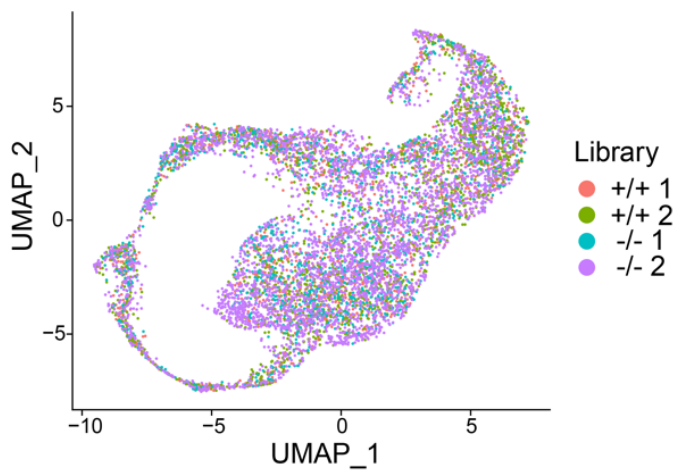

C.
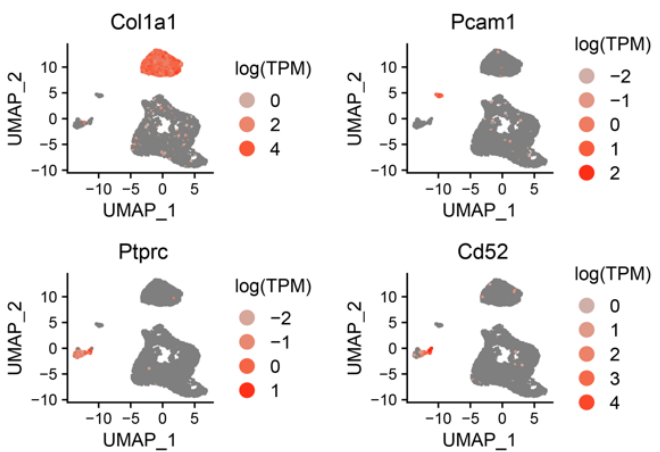

e.
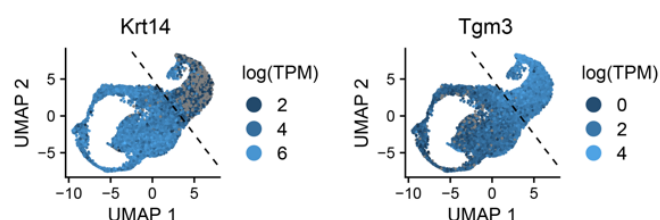

$\mathrm{Krt} 4$
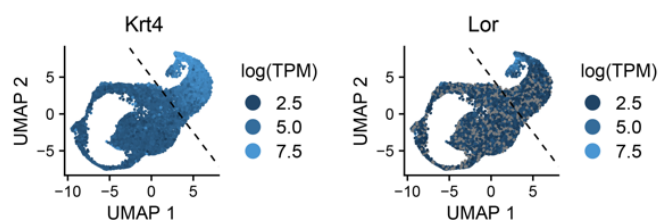

\section{Extended data Figure 6: single cell RNAseq of Notch1 mutant tissue}

a. qPCR recombination assay of Notch1 exon1 in peeled esophageal epithelium from Notch1 floxfflox mice induced once at the same dose as the one used for the sc-RNAseq and collected 4 weeks after induction. Data is shown in comparison to wild type tissue from the same qPCR assay. Mean \pm SEM, each dot represents a mouse, $n=3$ mice. Two-tailed unpaired Student's $t$-test. b. Esophageal epithelium was processed for single cell RNAseq as shown in Fig.4f. Box plots show transcripts per million (TPM) of Notch1 in the cells from each library ( $n=2$ biological replicates per genotype). c. UMAP plots show some markers that were used for cell type annotations of fibroblasts (Col1a1, top left), endothelial cells (Pcam1, top right) and immune cells (Ptprc, bottom left; Cd52, bottom right). See

Supplementary Note. d. UMAP plot shows an overlay of the cells annotated as keratinocytes from each library $(+/+1, n=1555 ;+/+2, n=1931 ;-/-1, n=1403 ;-/-2, n=3919)$. e. UMAP plots show markers of keratinocyte differentiation highlighting basal cells (Krt14, top left), differentiating cells (Tgm3, top right, $K r t 4$, bottom left) and cornified cells (Lor, bottom right). Dashed black line shows the threshold defining basal cells on the left and suprabasal cells on the right following Krt14 and Krt4 expression levels (Supplementary Note). AU, arbitrary unit. See Supplementary table 6. 
bioRxiv preprint doi: https://doi.org/10.1101/2021.06.18.448956; this version posted June 18, 2021. The copyright holder for this preprint

(which was not certified by peer review) is the author/funder, who has granted bioRxiv a license to display the preprint in perpetuity. It is made available under aCC-BY 4.0 International license.

\section{Figure 5}

a.
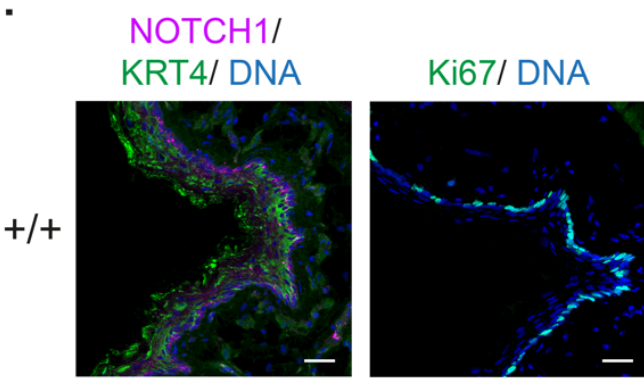

\section{LOR/}

KRT14/ DNA

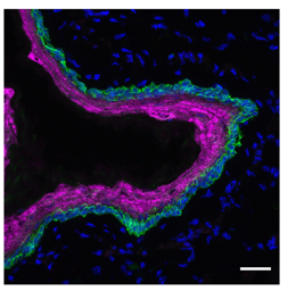

H\&E
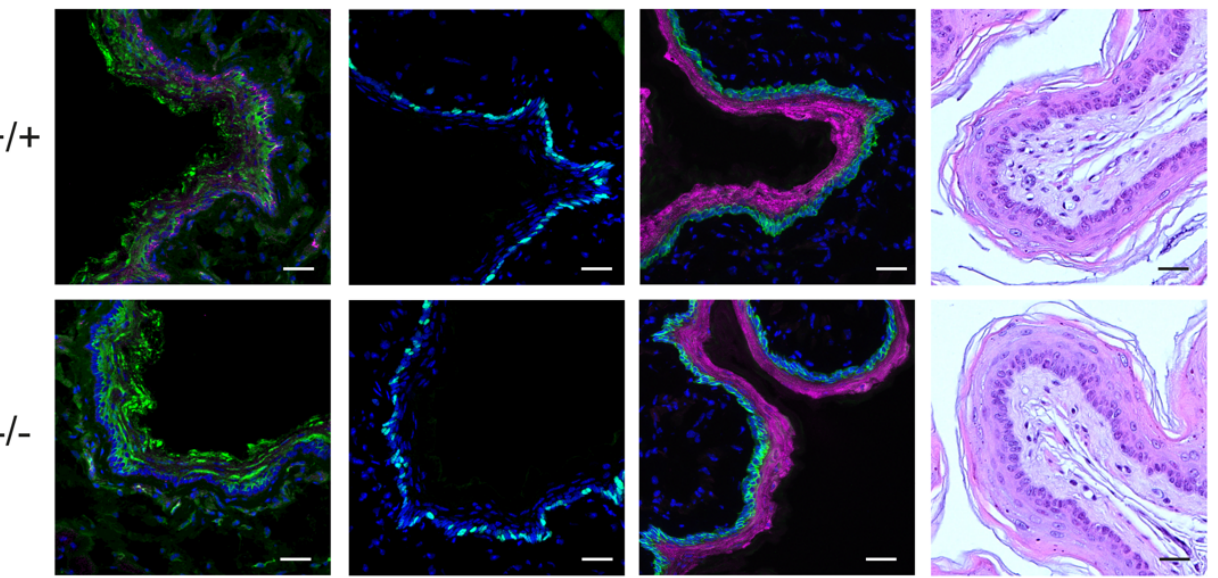

b.

C.
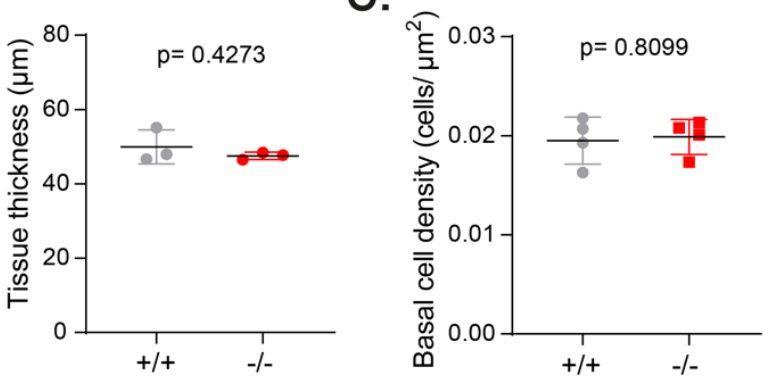

d.

e.

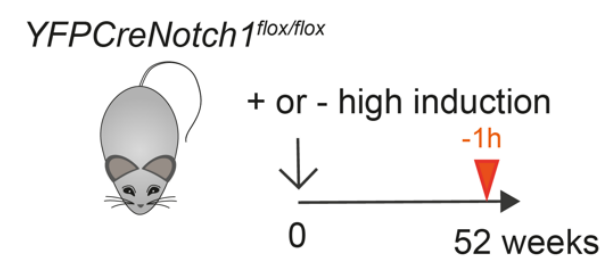

g.

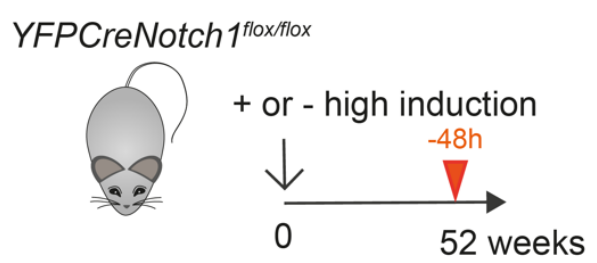

h.

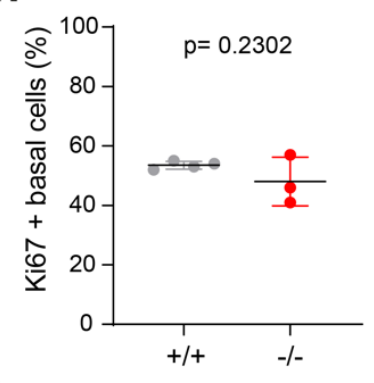

f.

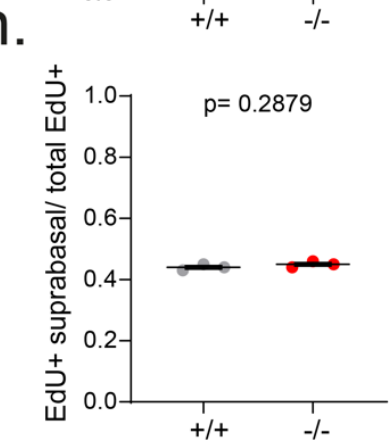




\section{Figure 5: Differentiation and homeostasis in Notch1 mutant mouse tissue}

a. Notch $1^{+/+}$and Notch $1^{\text {flox/flox }}$ mice were induced at high dose and aged as in (e) and (g) and tissue was collected. After sectioning, tissue was stained for basal cell marker KRT14, NOTCH1, proliferation marker Ki67, differentiation markers KRT4 and LOR and with Haematoxylin and eosin (H\&E). Images are representative from 3 mice of each genotype. Scale bars, $30 \mu \mathrm{m}$. b. Thickness of the epithelium was measured on H\&E scanned sections (Mean \pm SEM, each dot represents a mouse, $n=3$ mice). Twotailed unpaired Student's t-test. c. Epithelium basal cell density was measured on whole mount tissue. (Mean \pm SEM, each dot represents a mouse, $+/+, n=4097$ cells from 4 mice; $-/-, n=3964$ cells from 4 mice). Two-tailed unpaired Student's t-test. d. Proportion of proliferative basal cells were measured on sections stained for Ki67, KRT14 and DAPI. (Mean \pm SEM, each dot represents a mouse, $+/+, n=$ 1548 cells from 4 mice; -/-, $n=1129$ cells from 3 mice). Two-tailed unpaired Student's t-test. e, f. Highly induced or uninduced control Notch $1^{\text {flox/flox }}$ mice were aged for at least 52 weeks and injected with EdU $1 \mathrm{~h}$ before collection (e). Ratio of EdU positive basal cells on total number of basal cells was calculated (f) (Mean \pm SEM, each dot represents a mouse, $+/+, n=2754$ cells from 3 mice; -/-, $n=2565$ cells from 3 mice). Two-tailed, unpaired Student's t-test. $\mathbf{g}$, h. Highly induced or uninduced control Notch $1^{\text {flox/flox }}$ mice were aged for at least 52 weeks and injected with EdU 48h before collection (g). Ratio of EdU positive suprabasal cells. (Mean \pm SEM, each dot represents a mouse, $(+/+, n=2687$ EdU + cells from 3 mice; -/-, $n=2201 \mathrm{EdU}+$ cells from 3 mice). Two-tailed unpaired Student's t-test.

To augment these observations, we tracked cell dynamics by short term lineage tracing with EdU. 1h labelling indicated no significant difference in the proportion of $\mathrm{S}$ phase cells in Notch $1^{-/}$and control

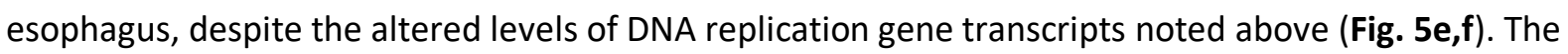
proportion of suprabasal EdU positive cells 48 hours post labelling was also similar in both strains, an observation that contrasts with Notch1-- cells in expanding clones. This suggested that once the esophagus has been fully colonized the behavior of Notch $1 \%$ progenitor cells reverts towards normal, sustaining a normal appearing tissue (Fig.5 g,h) ${ }^{16,19}$.

\section{Notch1 loss alters tumor growth}

NOTCH1 mutations are under strong positive selection in normal human esophagus and occupy $30 \%$ to $80 \%$ of the epithelium as early as middle-age but are only found in about $10 \%$ of esophageal squamous cell carcinomas (ESCC) ${ }^{1,3}$. This disparity hints at a role for wild type NOTCH1 in malignant transformation. We therefore explored the role of Notch1 in murine neoplasia. Notch1 wild type mice were treated with the mutagen Diethylnitrosamine (DEN), and Sorafenib, a multikinase inhibitor that increases cell turnover and promotes the formation of high grade dysplastic lesions ${ }^{29}$. After aging for 28 weeks, tissue was collected (Fig. 6a). Deep targeted sequencing of 73 cancer associated and Notch pathway genes was performed on dissected macroscopic tumors and a gridded array of normal epithelium (Fig.6a, Supplementary table 7).

The normal epithelium contained a high density of clones carrying protein altering mutations (Supplementary table 7). To determine which genes conferred a clonal advantage, we calculated the ratio of silent to protein altering mutations in each gene, $\mathrm{dN} / \mathrm{dS}^{5,30}$. Mutant genes under positive selection with a $\mathrm{dN} / \mathrm{dS}$ ratio significantly above $1(\mathrm{q}<0.05)$ were the Notch pathway genes Notch1, Notch2 and Adam10, plus Fat1, Trp53 and Arid1a, all of which are selected in normal human esophagus along with Ripk4 and Chuk (Supplementary table 7) ${ }^{1,19}$. 
bioRxiv preprint doi: https://doi.org/10.1101/2021.06.18.448956; this version posted June 18, 2021. The copyright holder for this preprint (which was not certified by peer review) is the author/funder, who has granted bioRxiv a license to display the preprint in perpetuity. It is made available under aCC-BY 4.0 International license.

In tumors, the most selected gene was the known mouse esophageal tumor driver Atp2a2, which is rarely mutated and not selected in normal epithelium (Extended data Fig. 7a,b. Supplementary table 7) ${ }^{31,32}$. Notch1 mutations were similar to the protein disrupting mutants in normal tissue with no evidence of activating mutations. However, Notch1 mutants were both less selected and less prevalent in tumors than in the adjacent tissue (Fig. 6b, c, Extended data Fig. 7a,b, Supplementary table 7). Immunostaining confirmed a higher proportion of cells staining positive for NOTCH1 and NICD1 in tumors than in the normal epithelium (Fig. 6d-g). These findings indicate Notch1 wild type cells are more likely to contribute to tumors than those carrying Notch1 mutants, consistent with the sequencing results in humans.

\section{Figure 6}

a.

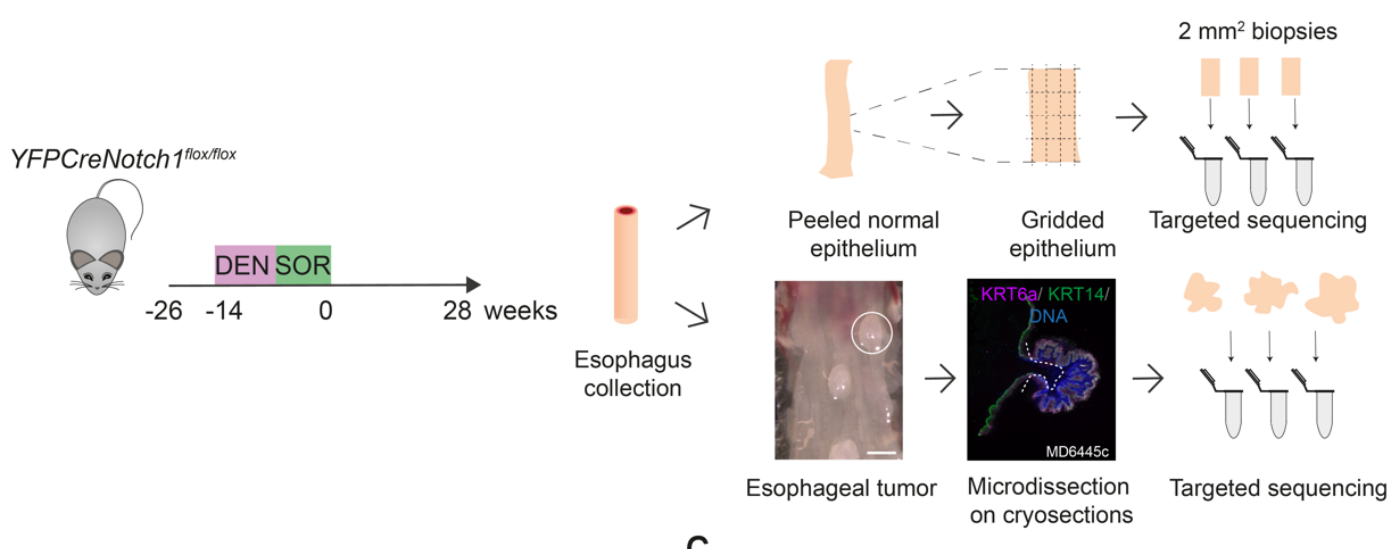

b.

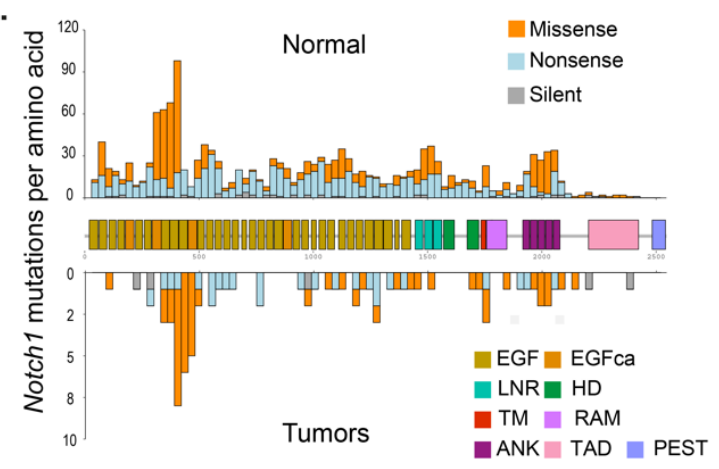

d.

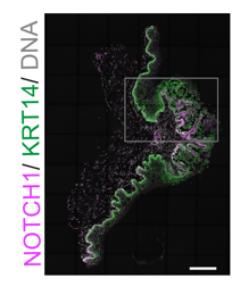

e.

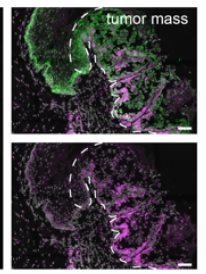

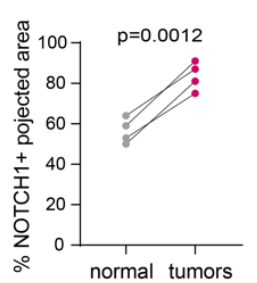

C.

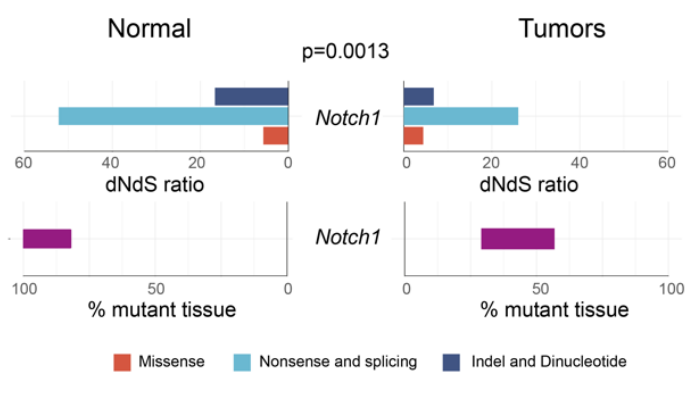

f.

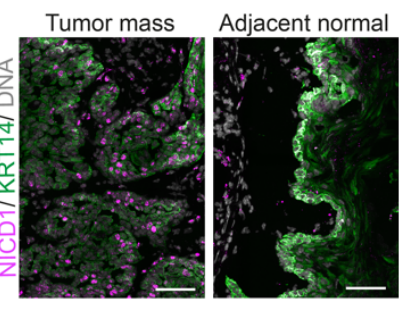

g.

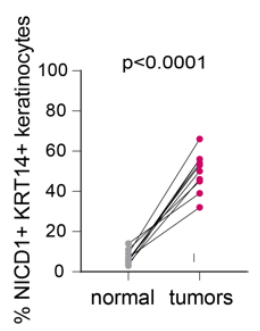




\section{Figure 6: Tumors retain functional Notch1 in carcinogenesis}

a. Uninduced YFPCreNotch $1^{\text {flox/flox }}$ mice were treated with DEN and Sorafenib. Tissue was collected 28 weeks after treatment. Tumors were dissected from underlying submucosa and normal epithelium was cut into a gridded array of $2 \mathrm{~mm}^{2}$ samples prior to targeted sequencing. Scale bar, $1 \mathrm{~mm}$. b. Number of Notch1 mutations per amino-acid is plotted by NOTCH1 protein domains in Notch1 $1^{+/}$ normal gridded biopsies (upper) and Notch $1^{+/+}$tumors (lower) (Normal, $\mathrm{n}=115$ biopsies from 6 mice; Tumors, $\mathrm{n}=17$ biopsies from 7 mice). c. $\mathrm{dN} / \mathrm{dS}$ ratio for Notch 1 mutations and proportion of Notch1 mutant tissue in normal epithelium ( $n=115$ biopsies from 6 mice) and tumors ( $n=17$ biopsies from 7 mice). Two-tailed p-value, likelihood ratio test of $d N / d S$ ratios ${ }^{1}$. $d$. Representative NOTCH1 (magenta) and KRT14 staining (green) in tumors and surrounding tissue, DNA is grey. Image typical of 10 tumors

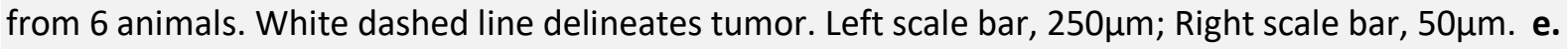
Proportion of NOTCH1+ staining area in normal epithelium and tumors from the same control animals (each dot represents a mouse, $n=40$ tumors from 4 mice). Two-tailed paired Student's t-test. $\quad$. Representative images showing nuclear NICD1 protein (magenta) in keratinocytes (KRT14, green) inside a tumor in comparison to the normal adjacent tissue. Image typical of 10 tumors from 6 animals. Scale bars, $50 \mu \mathrm{m}$. g. Proportion of KRT14 positive keratinocytes with nuclear NICD1 staining in tumors and surrounding epithelium in the same sections (each dot represents a tumor, $n=10$ tumors from 6 mice). Two-tailed paired Student's t-test. See Supplementary table 7.

To test if wild type Notch1 is required for tumor formation we used a high induction protocol to delete one or both alleles in the entire esophageal epithelium of Notch $1^{\text {flox/flox }}$ and Notch ${ }^{+/ f l o x}$ mice prior to treatment with DEN and Sorafenib. Uninduced littermates were used as wild type controls (Fig. 7a). Detailed microscopic and macroscopic analysis of the tissue revealed that the density of tumors was similar in all three genotypes, arguing Notch 1 is not required for tumor formation (Fig.7 b,c). However, tumors were significantly smaller in Notch $1^{-/}$, compared to Notch $1^{+/}$and Notch $1^{+/+}$animals (Fig. 7d,e, Extended data Fig. 7c). NOTCH1 immunostaining confirmed the loss of Notch1 expression in tumors from induced Notch $1^{\text {flox } / f l o x}$ mice. (Extended data Fig. 7d). Immunostaining for differentiation markers (LOR, KRT14) revealed that the tumors had similar staining for proliferation (Ki67), apoptosis (cleaved caspase 3), endothelial cell (CD31) and immune cell (CD45) markers in lesions from Notch1\% and Notch $^{+/+}$esophagus (Fig. 7e, Extended Data Fig. 7d-f). The cell-cell adhesion protein CDH1 (Ecadherin) is down regulated in human ESCC and loss of CDH1 signaling promotes tumorigenesis in mice ${ }^{33}$. Interestingly, Notch1 wild type tumors displayed focal loss of $\mathrm{CDH} 1$ expression, a feature that was not seen in the Notch $1^{\%}$ tumors (Fig.7 f,h). 
bioRxiv preprint doi: https://doi.org/10.1101/2021.06.18.448956; this version posted June 18, 2021. The copyright holder for this preprint

(which was not certified by peer review) is the author/funder, who has granted bioRxiv a license to display the preprint in perpetuity. It is made available under aCC-BY 4.0 International license.

\section{Figure 7}

a.

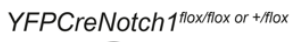

YFPCreNotch $1^{\text {floxfllox or +fflox }}$

e.

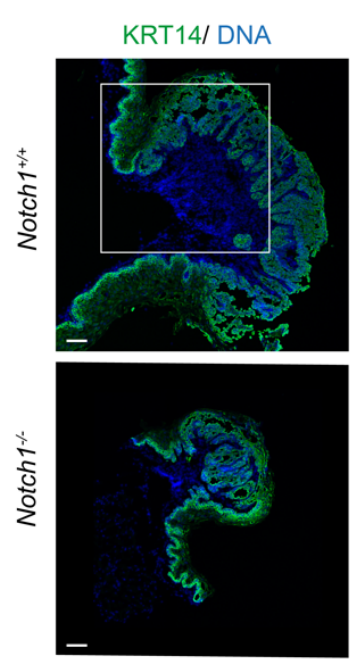

i.

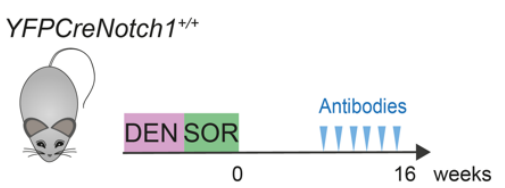

b.

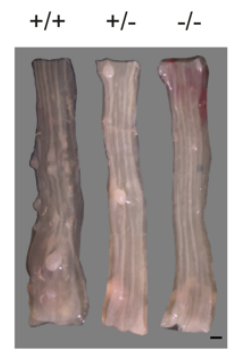

f.
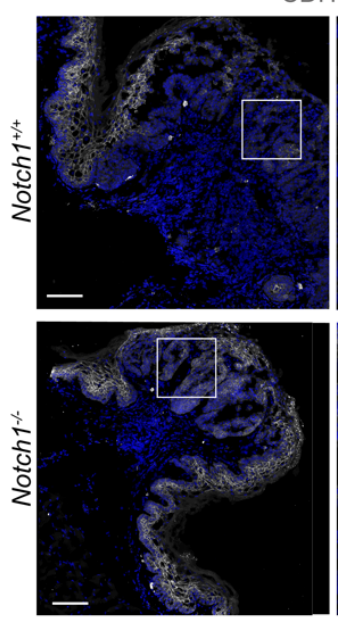

j.

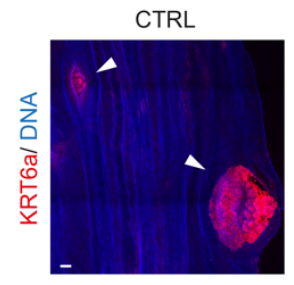

C.

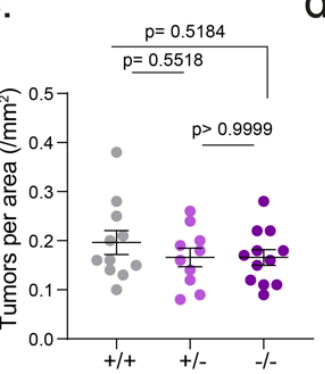

DH1/ DNA
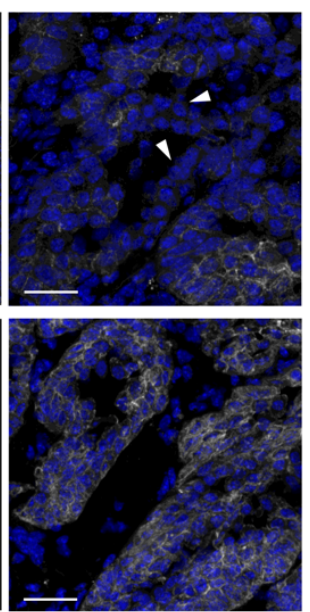

anti-NRR1

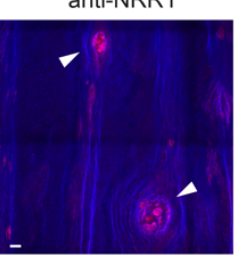

d.

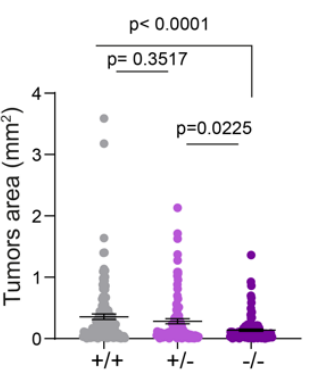

g.

h.

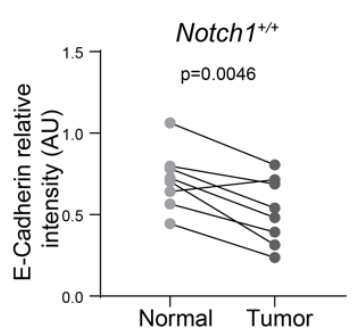

k.
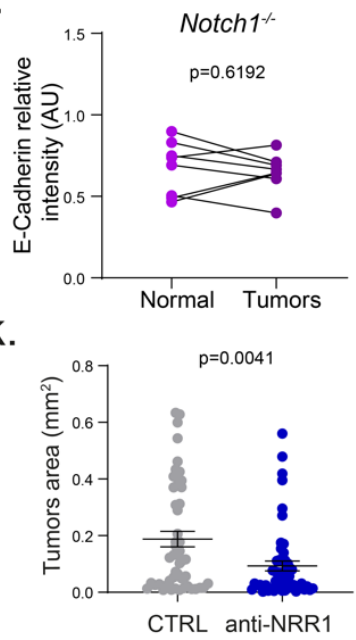
bioRxiv preprint doi: https://doi.org/10.1101/2021.06.18.448956; this version posted June 18, 2021. The copyright holder for this preprint (which was not certified by peer review) is the author/funder, who has granted bioRxiv a license to display the preprint in perpetuity. It is made available under aCC-BY 4.0 International license.

\section{Figure 7: Tumor growth is reduced by Notch1 inactivation}

a. Highly induced YFPCreNotch $1^{+f f l o x}$ and YFPCreNotch $1^{\text {floxfflox }}$ mice or uninduced control mice were treated with DEN and Sorafenib and aged for 28 weeks. Mouse numbers for $\mathbf{b}-\mathbf{d}, \operatorname{Notch}^{+/+}, \mathrm{n}=11$; Notch1 $1^{+-} \mathrm{n}=10 ;$ Notch $^{-1}, \mathrm{n}=12$. b. Esophagi from treated control, heterozygous, homozygous mice collected at experimental end point. Images are representative of each group. Scale bar, $1 \mathrm{~mm}$. c. Tumor density per genotype. Mean $\pm S E M$, each dot represents a mouse, number of mice as shown in b. One-way ANOVA; adjusted $p$ values from Tukey's multiple comparisons test. $\mathbf{d}$. Tumor areas per genotype. Mean \pm SEM, each dot represents a tumor. One-way ANOVA; adjusted $p$ values from Tukey's multiple comparisons test. e, f. Tumors from Notch $1^{+/+}$and $\mathrm{Notch}^{\%}$ mice were stained for immunostained for KRT14 (green) (e), or E-cadherin (CDH1, white) (f). Right hand images in $\mathbf{f}$ are magnified views of areas shown by a white square. White arrows indicate areas with reduced CDH1 staining. Images are representative of $n=8$ stained tumors from 6 mice of each genotype. Scale bars, $100 \mu \mathrm{m}$ (e, f left panel) and $30 \mu \mathrm{m}$ (f right panel). g,h. Mean E-cadherin intensity relative to DNA staining was quantified inside the tumor mass and compared to the corresponding adjacent normal tissue in wild type $(\mathrm{g})$ and Notch $1^{-1}(\mathrm{~h})$ tissues ( $\mathrm{n}=8$ tumors from 6 mice for each genotype). Two tailed paired Student's t-test. i. YFPCreNotch $1^{+/+}$mice were treated with DEN/SOR and aged for 9 weeks. Mice were treated with anti-NRR1.1E3 or with control anti-Ragweed antibody (CTRL) for 6 weeks before collection. j. Representative tumors marked by KRT6a staining (red) are shown with white arrows in esophageal epithelium from control and anti-NRR1.1E3 treated mice. Scale bars: $100 \mu \mathrm{m}$. k. Quantification of the tumor area (Mean $\pm S E M$, each dot represents a tumor, $n=4$ mice per group). $P$ values from two-tailed unpaired Student's t-test. AU, arbitrary unit. 
bioRxiv preprint doi: https://doi.org/10.1101/2021.06.18.448956; this version posted June 18, 2021. The copyright holder for this preprint

(which was not certified by peer review) is the author/funder, who has granted bioRxiv a license to display the preprint in perpetuity. It is made available under aCC-BY 4.0 International license.

\section{Extended data Figure 7}

a.

C.

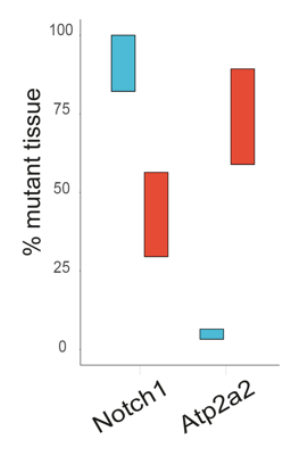

$\square$ normal

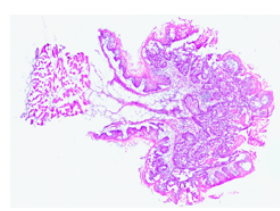

Notch 1 - tumors
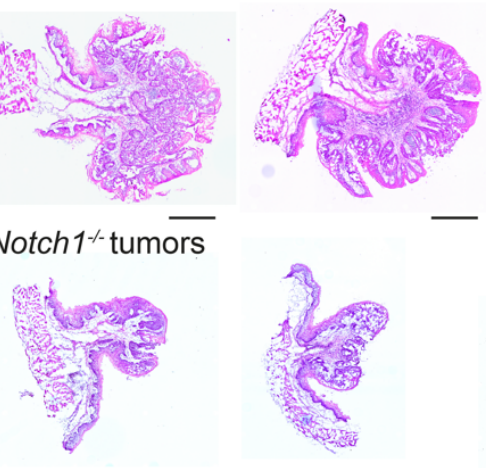
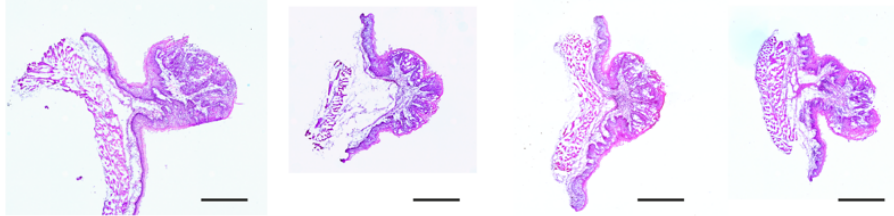

Notch1 $\mathrm{am}=\mathrm{a}$

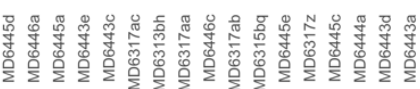
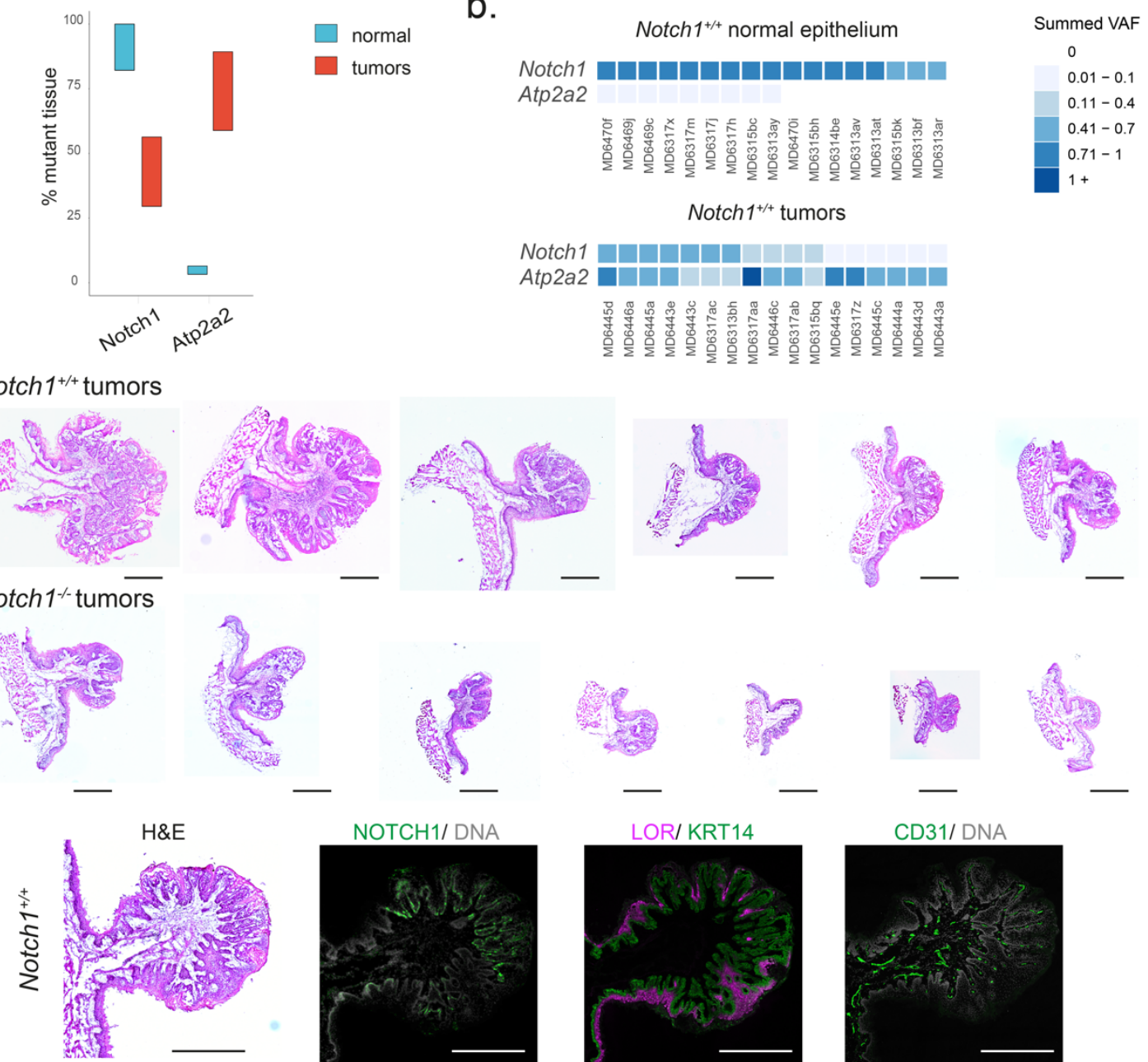

d.
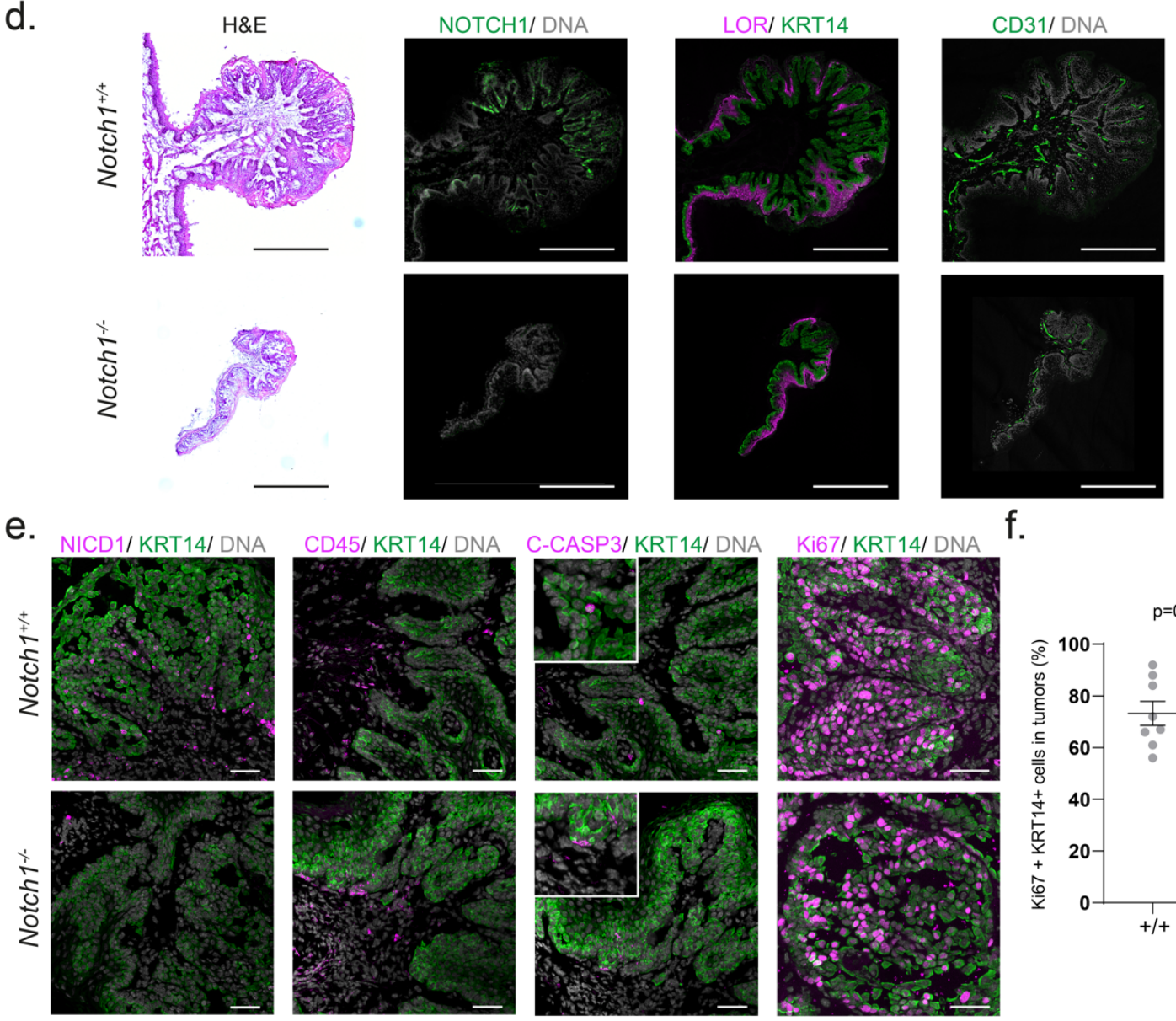

f. 


\section{Extended Data Figure 7: Genetic and histological characterization of mouse esophageal tumors}

a. As in Fig.6a, mouse esophageal tissue and tumors from carcinogenesis assay were collected 28 weeks after treatment and processed for targeted sequencing. Proportion of tissue mutant for Notch1 and Atp2a 2 in tumors and in normal epithelium. Estimates are from sum of VAFs of non-synonymous mutations for Notch1 and Atp2a2 ( $n=115$ normal epithelial biopsies from 6 mice, n=17 tumors from 7 mice; Supplementary Note). b. Summed VAF of protein altering mutations in the indicated genes is shown for randomly picked and representative normal gridded tissues (upper, n=17 out of 115 biopsies) and each 17 sequenced tumors (lower). c. Hematoxylin and eosin (H\&E) staining of tumors from uninduced wild type and induced Notch $1 \%$ mice treated with DEN and Sorafenib and aged for 28 weeks as shown in Fig. 6. Pictures are representative of $n=19$ wild type and $n=11$ Notch $^{\%}$ stained tumors. Scale bars, $500 \mu \mathrm{m}$. d, e. Notch $1^{+/}$and Notch $1^{\%-}$ tumors were sectioned and stained, from left to right, (d) for H\&E, NOTCH1, differentiation markers (KRT14 and LOR), endothelial cell marker CD31, (e) active NOTCH1 (NICD1), immune cell marker CD45, for apoptosis marker cleaved Caspase 3 and proliferation marker Ki67. Images are representative of $n=10$ wild type tumors and $n=11$ Notch $1^{-}$ tumors for all immunostainings and cleaved Caspase 3 ( $n=4$ tumors). Scale bars in $d, 500 \mu m$, in e, 50 $\mu \mathrm{m}$. f. Percentage of Ki67 positive, KRT14+ keratinocytes within tumors from Notch $1^{+/+}$and $\mathrm{Notch}^{-/-}$ esophagus. Mean \pm SEM, each dot represents a tumor, $n=8$ tumors from 5 Notch $1^{+/+}$mice and $n=9$ tumors from 6 Notch $1^{-}$mice. Two-tailed unpaired Student's t-test.

These observations argue that Notch1 promotes tumor growth. To further test this hypothesis, we treated wild type mice with a neutralizing antibody targeting the negative regulatory region (antiNRR1.1E3) of NOTCH1, blocking its cleavage and NICD1 release ${ }^{34}$. A short-term analysis revealed that the antibody was able to reduce levels of cleaved NOTCH1 in esophageal epithelium, abolish nuclear NICD1 immunostaining and normalize altered levels of multiple transcripts dysregulated by loss of Notch1 such as Sox9, Igfbp3, Gli2, Tgm2 and Adam8 in vivo (Extended data Fig. 8a-e). To determine the dosage of anti-NRR1.1E3 that would both inhibit NOTCH1 in esophageal epithelium and was well tolerated over an extended period of treatment we performed a clonal competition assay. Notch $1^{-}$ clones were induced in YFPCReNotch $1^{\text {flox/flox }}$ mice a week before a three-week course of antibody treatment (Extended data Fig. 8f). Effective blockade NOTCH1 signaling in the epithelium eliminates the competitive advantage of Notch $1 \%$ clones over wild type cells, so mutant and wild type cells compete neutrally and mutant clone size will be reduced compared with controls (Extended data Fig. 8g). $10 \mathrm{mg} / \mathrm{kg}$ Anti-NRR1.1E3 was both tolerated and significantly reduced in Notch $1 \%$ clone size ( $p<0.0001$, One-way ANOVA; Tukey's multiple comparisons test) (Extended data Fig. $8 \mathrm{~h}, \mathbf{i})$.

Wild type mice were given DEN and Sorafenib after which tumors were allowed to develop for 9 weeks. Animals were then treated with anti-NRR1.1E3 or control antibody at $10 \mathrm{mg} / \mathrm{kg}$ for 6 weeks (Fig. 7i). The anti-NOTCH1 antibody treatment resulted in significant reduction in tumor size compared with controls, indicating active Notch signaling promotes the growth of established lesions (Fig.7 j-k). 


\section{Extended data Figure 8}

a.

$\mathrm{C} 57 \mathrm{BI} / 6$

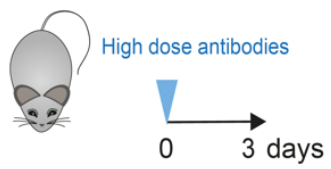

b.

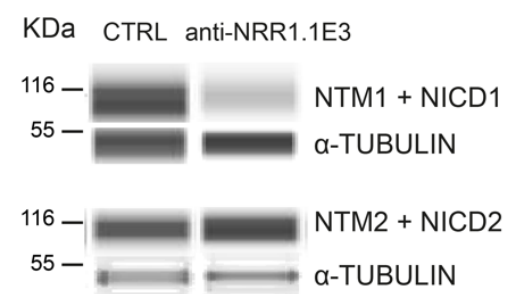

C. $z$

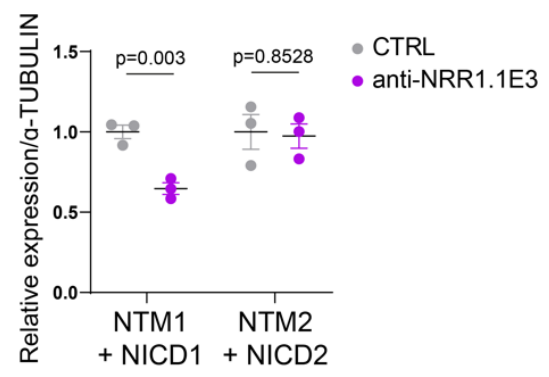

d.

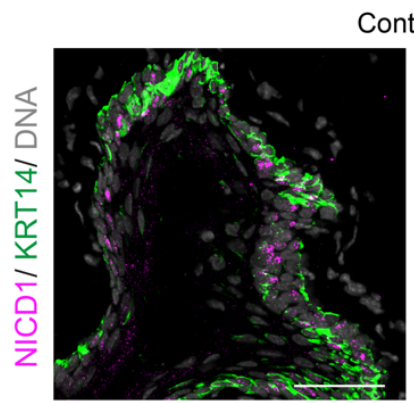

Control
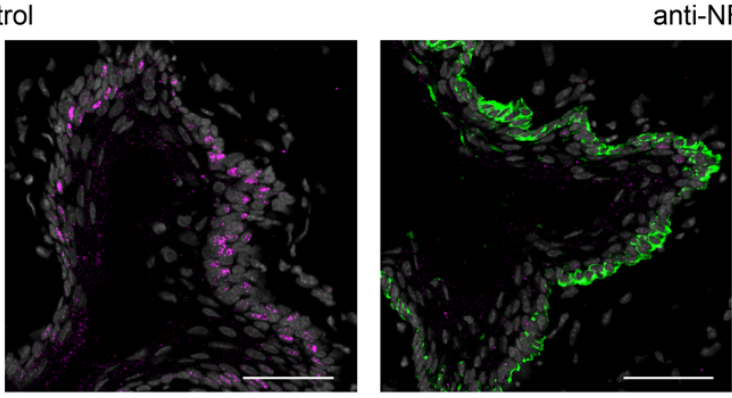

anti-NRR1.1E3

e.
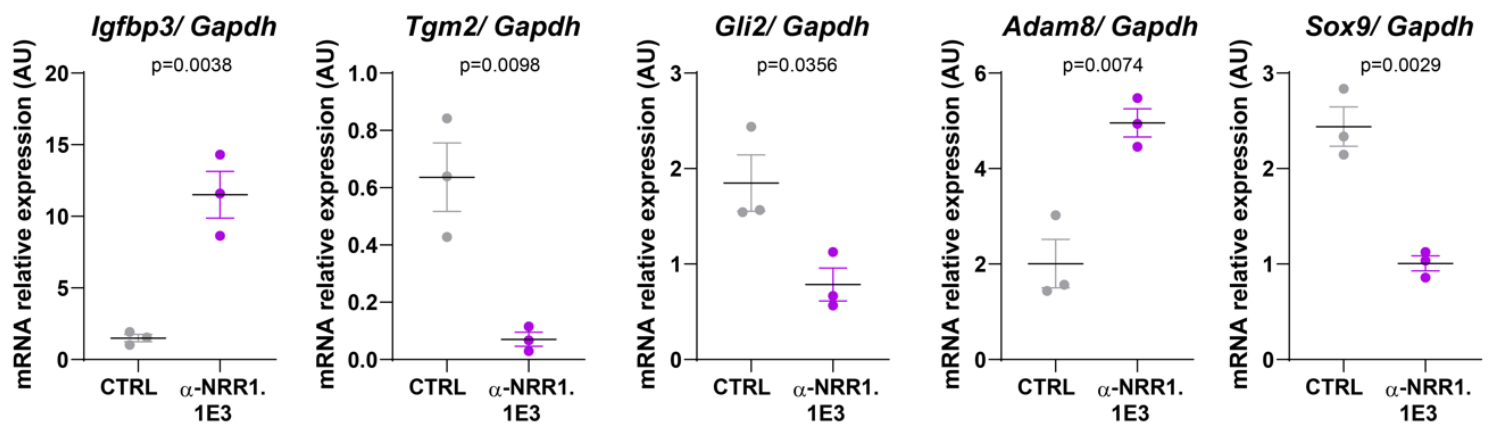

f.

g.
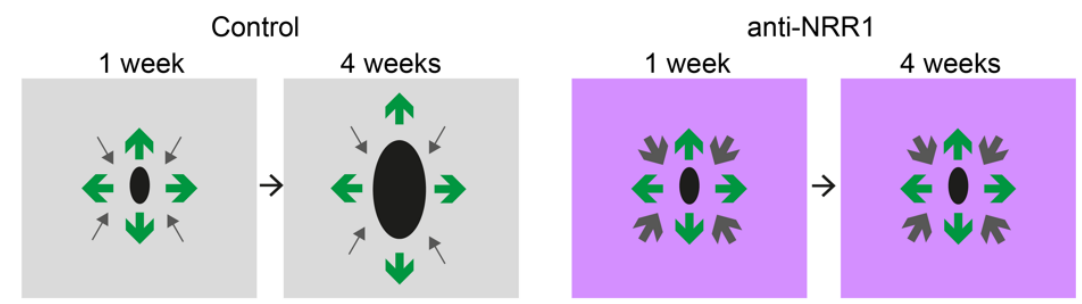

h.

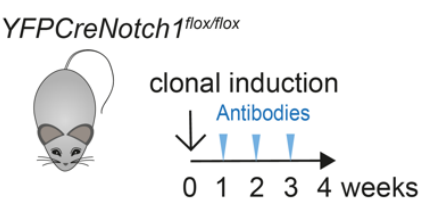

1 week

i.
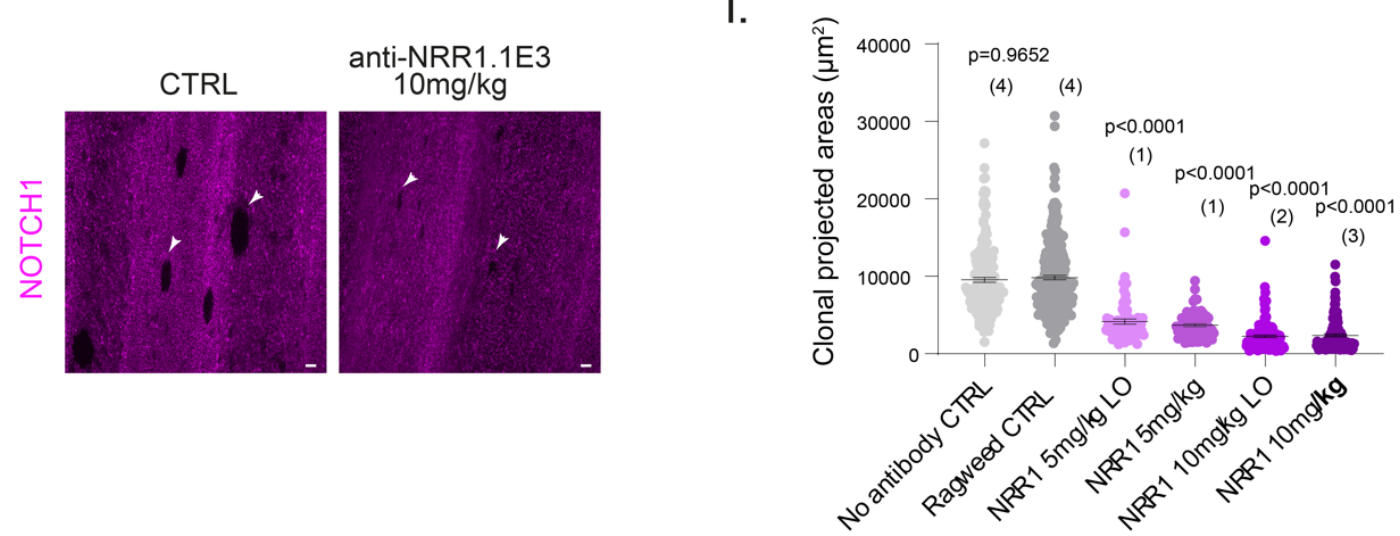


\section{Extended data figure 8: anti-NRR1 antibodies inhibit NOTCH1 signaling in vivo}

a. Protocol for b-e. C57BI/6 wild type mice were treated using neutralizing anti-NRR1.1E3 or antiRagweed control antibodies (CTRL) at high dose $(25 \mathrm{mg} / \mathrm{kg}$ ) three days before tissue collection $\mathbf{b}$. Immune Capillary Electrophoresis assay for the cleaved transmembrane and intracellular regions of NOTCH1 (NTM1 + NICD1, Extended data Fig1a) and NOTCH2 (NTM2 + NICD2), and $\alpha$-TUBULIN proteins from esophageal epithelium of mice in a. c. Quantification of the relative expression of the cleaved transmembrane/intracellular regions of NOTCH1 and NOTCH2 (NTM + NICD) normalized on $\alpha-$ TUBULIN expression in the indicated conditions. Mean \pm SEM, each dot represents a mouse, $n=3$ mice. Two-tailed unpaired Student's t-test. d. Representative pictures of immunostaining for NICD1 (magenta), KRT14 (green) and nuclei (grey) in esophageal cryosections of mice treated with control or anti-NRR1.1E3 antibodies $(n=3)$. Scale bars, $30 \mu \mathrm{m}$. e. Plots show RT-qPCR measurement of Notch1 loss of function marker transcripts identified in the bulk RNAseq (Supplementary table 5) in control and anti-NRR1.1E3 treated samples. Mean \pm SEM, each dot represents a mouse, $n=3$. Two-tailed unpaired Student's t-test. f. Protocol for g-i. YFPCreNotch $1^{\text {flox/flox }}$ mice were induced at clonal density and antibody treatment started one week later. Mice were dosed with anti-NRR1.1E3 or control antibodies for 3 weeks before esophageal collection. $g$. Principle of assay shown in $\mathbf{f}$ to measure the efficacy of the long-term treatment. As expansion of clones is subject to the fitness of the neighbor cells ${ }^{19}$, induced Notch $1 \%$ cells treated with control antibody one week after induction have higher fitness (green arrows) than surrounding wild type cells (grey arrows) and clones expand to a bigger size until collection at 4 weeks. If the anti-NRR1.1E3 treatment blocks NOTCH1 signaling, the surrounding cells have a fitness identical to the induced Notch $1^{-}$cells and the expansion of Notch $1^{-}$ clones will be halted. $\mathbf{h}$. Representative images of NOTCH1 negative clones in esophageal epithelia treated with control or anti-NRR1.1E3 antibody at $10 \mathrm{mg} / \mathrm{kg}$ and collected one week after the last dose. Images are representative of entire esophagus from 3 mice. Scale bars, $50 \mu \mathrm{m}$. i. Quantification of area of clones negative for NOTCH1 staining. Mean \pm SEM, each dot represents a clone. Number of mice analyzed per dose are indicated in brackets. One-way ANOVA; Tukey's multiple comparisons test, adjusted p-values shown against Ragweed control condition. AU, arbitrary unit. LO, loading.

\section{Discussion}

These results shed light on the disparity in the prevalence of NOTCH1 mutations in normal esophageal epithelium and tumors. A consequence of normal progenitor behavior is that most clones carrying mutations that do not alter progenitor cell behavior are lost by differentiation, making biallelic loss unlikely. Mutations reducing the function of one Notch1 allele confer a competitive advantage on mutant progenitors, giving them a greatly enhanced probability of founding persistent, expanding clones. As the heterozygous mutant population grows, the likelihood that the remaining allele will be lost increases. When this happens, it confers a further increase in fitness. Over time, the result is extensive colonization of the epithelium by biallelic mutants, as wild type and heterozygous cells are displaced.

Once an area has been colonized by biallelic Notch1 mutants, the behavior of mutant progenitors reverts towards that of wild type progenitors so that the epithelium remains histologically normal in both humans and mice. Loss of Notch1 does impact transcription of genes such as MCM proteins implicated in DNA replication and transcripts that regulate squamous epithelial morphogenesis, but functional and histological analyses indicate the epithelium is not substantially perturbed by these 
changes. These observations explain the remarkably high prevalence of Notch1 mutations in normal appearing esophagus.

In contrast to normal epithelium, the loss of Notch1 has a substantial phenotypic effect in carcinogenesis. Notch1 is less mutated in tumors than wild type epithelium and its loss impairs tumor growth, an effect recapitulated by NOTCH1 neutralizing antibodies. How might wild type Notch1 promote tumor growth? Our transcriptional analysis suggests some potential mechanisms. Examples include transcripts of genes that promote growth and invasiveness in ESCC cells, such as Nrp2, Cdk14 and Sox9 which are more highly expressed in Notch1 wild type than null esophageal cells ${ }^{35-37}$. Conversely, transcription of Cldn7 which represses ESCC invasiveness is upregulated in Notch 1 - cells 38,39. Our study opens the way for further characterization of the pleotrophic effects of Notch1 in esophageal tumors.

We conclude that Notch1 illustrates how inactivating mutations in the same gene can drive clonal expansion in normal tissue but inhibit tumor growth. Our results further raise the possibility that blockade of NOTCH1 may have therapeutic potential in early esophageal neoplasia by reducing the growth of premalignant tumors. Multiple NOTCH1 inhibitors are in preclinical and clinical development and further investigation of their role in esophageal neoplasia seems warranted.

\section{Competing interests}

The authors declare no competing interests.

\section{Author contributions}

E.A., J.C.F, and P.H.J designed experiments. E.A. and J.C.F performed experiments. E.A., S.C.D, M.W.J.H, S.H.O and R.S. analysed experimental and sequencing data. S.C.D. and M.G. created pipelines for copy number and scRNA-seq analyses. M.W.J.H. and B.A.H. performed clone simulations. C.W.S. provided neutralizing antibodies. E.A, S.C.D, M.W.J.H. and P.H.J wrote the paper. M.G, B.AH. and P.H.J supervised the research. All authors discussed the results and commented on the manuscript.

\section{Acknowledgements}

This work was supported by grants from the Wellcome Trust to the Wellcome Sanger Institute (098051 and 296194) and Cancer Research UK Programme Grants to P.H.J. (C609/A17257 and C609/A27326). E.A. benefited from the award of a Postdoc fellowship, 2016-21, from the Wellcome Sanger Institute. B.A.H. and M.W.J.H. are supported by the Medical Research Council (Grant-in-Aid to the MRC Cancer unit grant no. MC_UU_12022/9 and NIRG to B.A.H. grant no. MR/S000216/1). M.W.J.H. acknowledges support from the Harrison Watson Fund at Clare College, Cambridge. B.A.H. acknowledges support from the Royal Society (grant no. UF130039). S.C.D. benefited from the award of an ESPOD fellowship, 2018-21, from the Wellcome Sanger Institute and the European Bioinformatics Institute EMBL-EBI.

We thank Esther Choolun, Tom Metcalf and Sanger RSF facility for technical support with animal research. We thank Yvette Hooks, Claire Hardy, Calli Latimer and staff from the CASM support laboratory for technical support with histology and sequencing. 


\section{Legends for videos and tables}

\section{Supplementary tables}

The supplementary tables include source data for all figures

Supplementary Table 1: List of genes in the targeted sequencing experiments performed in this study Supplementary Table 2a: Targeted sequencing of Human esophageal epithelium micro-biopsies; Complete mutation calling with Shearwater (part1) and from Caveman and Pindel pipelines (Part2)

Supplementary Table $2 \mathrm{~b}$ : $\mathrm{dNdS}$ analysis of the Human micro-biopsy sequencing

Supplementary Table 2c: NOTCH1 mutations summary in Human esophageal epithelium microbiopsies

Supplementary table 3: Human samples metadata

Supplementary table 4a: Notch1 mutations and CNLOH analysis in NOTCH1 stained Notch1+/microbiopsies

Supplementary table 4b: Mutation calling in Notch1+/- micro-biopsies

Supplementary Table 5a: Differentially expressed genes in Notch1 mutant mouse esophageal epithelium

Supplementary Table 5b: EnrichGO analysis in Notch1-/- vs Notch1+/+ and Notch1+/- vs Notch1+/+ esophageal epithelium

Supplementary Table 5c: Differential expression analysis of canonical Notch targets and keratinocyte function related genes in Notch1-/- mouse tissue

Supplementary table 6: Single-cell transcriptomic analysis of Notch1-/- and Notch1+/+ mouse esophageal epithelium

Supplementary table 7a: Mutation calling from targeted sequencing of DEN SOR treated esophageal epithelium

Supplementary table 7b: Mutation calling from targeted sequencing of esophageal tumors 28 weeks post-DEN and Sorafenib treatment

Supplementary Table 7c: dNdS analysis in normal mouse esophageal epithelium after DEN SOR treatment

Supplementary Table 7d: $d N d S$ analysis in mouse esophageal tumors

Supplementary table 8: Antibodies used for immunostaining 
bioRxiv preprint doi: https://doi.org/10.1101/2021.06.18.448956; this version posted June 18, 2021. The copyright holder for this preprint (which was not certified by peer review) is the author/funder, who has granted bioRxiv a license to display the preprint in perpetuity. It is made available under aCC-BY 4.0 International license.

\section{Video1:}

In silico simulations showing clonal expansion in YFPCreNotch1 mutant mouse model over a period of 30 days after clonal induction. The top video (+/+) shows YFP+ Notch $1^{+/+}$cells (green) expanding within a wild type epithelium (magenta). The middle video (+/-) shows YFP+ Notch $1^{+/+}$and YFP+ Notch $1^{+/}$ clones (green) as well as YFP- Notch1 ${ }^{+/}$(purple) evolving in a wild type tissue (magenta). The bottom video (-/-) shows YFP+ Notch1 ${ }^{+/+}$and YFP+ Notch1 ${ }^{-/}$clones (green) as well as YFP-Notch1 ${ }^{-/}$(blue) evolving in a wild type tissue (magenta). 2-dimensional Wright-Fisher style model of clone dynamics in the esophagus and parameter fitting are described in Extended data Fig.4 and Supplementary Note.

\section{Video2:}

In silico simulations showing the effect of Notch1 haploinsufficiency on the appearance of spontaneous Notch $1 \%$ clone in the epithelium. The top video shows the scenario of Notch1 haploinsufficiency ( $\mathrm{HI})$, using the best fit fitness parameter to the experimental analysis of Notch $1^{+/}$clones. The bottom video shows the scenario of Notch $1^{+/-}$fitness being neutral (haplosufficiency, HS). Notch $1^{+-}$clones are shown in purple, Notch $1 \%$ emerging by selection from Notch $1^{+/-}$cells are shown in red and wild type cells are shown in light blue. Simulation results are shown over a period of 5000 days after the scattered induction Notch1+- clones. In both cases, the best fit fitness parameter for Notch $1 \%$ fitness was used. See Extended data Fig.4 and Supplementary Note. 


\section{References}

1 Martincorena, I. et al. Somatic mutant clones colonize the human esophagus with age. Science (New York, N.Y.) 362, 911-917, (2018).

2 Yokoyama, A. et al. Age-related remodelling of oesophageal epithelia by mutated cancer drivers. Nature 565, 312-317, (2019).

3 Consortium, T. Integrated genomic characterization of oesophageal carcinoma. Nature 541, 169-175, (2017).

4 Blokzijl, F. et al. Tissue-specific mutation accumulation in human adult stem cells during life. Nature 538, 260-264, (2016).

5 Martincorena, I. et al. Tumor evolution. High burden and pervasive positive selection of somatic mutations in normal human skin. Science (New York, N.Y.) 348, 880-886, (2015). Aster, J. C., Pear, W. S. \& Blacklow, S. C. The Varied Roles of Notch in Cancer. Annual Review of Pathology: Mechanisms of Disease 12, 245-275, (2017).

7 Lloyd-Lewis, B., Mourikis, P. \& Fre, S. Notch signalling: sensor and instructor of the microenvironment to coordinate cell fate and organ morphogenesis. Current Opinion in Cell Biology 61, 16-23, (2019).

8 Kovall, R. A., Gebelein, B., Sprinzak, D. \& Kopan, R. The Canonical Notch Signaling Pathway: Structural and Biochemical Insights into Shape, Sugar, and Force. Developmental Cell 41, 228241, (2017).

9 Zhang, Y. et al. 3D Modeling of Esophageal Development using Human PSC-Derived Basal Progenitors Reveals a Critical Role for Notch Signaling. Cell stem cell 23, 516-529.e515, (2018). Lubin, D. J., Mick, R., Shroff, S. G., Stashek, K. \& Furth, E. E. The notch pathway is activated in neoplastic progression in esophageal squamous cell carcinoma. Human Pathology 72, 66-70, (2018).

11 Natsuizaka, M. et al. Interplay between Notch1 and Notch3 promotes EMT and tumor initiation in squamous cell carcinoma. Nature Communications 8, 1758, (2017).

12 Sawangarun, W. et al. Loss of Notch1 predisposes oro-esophageal epithelium to tumorigenesis. Experimental Cell Research 372, 129-140, (2018).

13 Doupe, D. P. et al. A single progenitor population switches behavior to maintain and repair esophageal epithelium. Science (New York, N.Y.) 337, 1091-1093, (2012).

14 Piedrafita, G. et al. A single-progenitor model as the unifying paradigm of epidermal and esophageal epithelial maintenance in mice. Nature Communications 11, 1429, (2020).

15 Murai, K. et al. Epidermal Tissue Adapts to Restrain Progenitors Carrying Clonal p53 Mutations. Cell stem cell 23, 687-699.e688, (2018).

16 Alcolea, M. P. et al. Differentiation imbalance in single oesophageal progenitor cells causes clonal immortalization and field change. Nature cell biology 16, 615-622, (2014).

17 Radtke, F. et al. Deficient T cell fate specification in mice with an induced inactivation of Notch1. Immunity 10, 547-558, (1999).

18 Lowell, S., Jones, P., Le Roux, I., Dunne, J. \& Watt, F. M. Stimulation of human epidermal differentiation by delta-notch signalling at the boundaries of stem-cell clusters. Curr Biol 10, 491-500, (2000).

19 Colom, B. et al. Spatial competition shapes the dynamic mutational landscape of normal esophageal epithelium. Nature Genetics 52, 604-614, (2020).

20 Rand, M. D. et al. Calcium depletion dissociates and activates heterodimeric notch receptors. Molecular and cellular biology 20, 1825-1835, (2000).

21 Schymkowitz, J. et al. The FoldX web server: an online force field. Nucleic Acids Res 33, W382$388,(2005)$.

22 Fowler, J. C. et al. Selection of oncogenic mutant clones in normal human skin varies with body site. Cancer Discovery, CD-20-1092, (2020).

23 Gordon, W. R. et al. Structure of the Notch1-negative regulatory region: implications for normal activation and pathogenic signaling in T-ALL. Blood 113, 4381-4390, (2009). 

lymphoproliferative disorders. Current opinion in hematology 23, 362-370, (2016).

Tate, J. G. et al. COSMIC: the Catalogue Of Somatic Mutations In Cancer. Nucleic Acids Res 47, D941-d947, (2019).

26 Lee, J., Basak, J. M., Demehri, S. \& Kopan, R. Bi-compartmental communication contributes to the opposite proliferative behavior of Notch1-deficient hair follicle and epidermal keratinocytes. Development (Cambridge, England) 134, 2795-2806, (2007).

27 Blanpain, C., Lowry, W. E., Pasolli, H. A. \& Fuchs, E. Canonical notch signaling functions as a commitment switch in the epidermal lineage. Genes \& development 20, 3022-3035, (2006).

28 Stuart, T. et al. Comprehensive Integration of Single-Cell Data. Cell 177, 1888-1902.e1821, (2019).

29 Frede, J., Greulich, P., Nagy, T., Simons, B. D. \& Jones, P. H. A single dividing cell population with imbalanced fate drives oesophageal tumour growth. Nature cell biology 18, 967-978, (2016).

30 Martincorena, I. et al. Universal Patterns of Selection in Cancer and Somatic Tissues. Cell 171, 1029-1041.e1021, (2017).

31 Liu, L. H., Boivin, G. P., Prasad, V., Periasamy, M. \& Shull, G. E. Squamous Cell Tumors in Mice Heterozygous for a Null Allele of Atp2a2, Encoding the Sarco(endo)plasmic Reticulum Ca2+ATPase Isoform 2 Ca2+Pump. Journal of Biological Chemistry 276, 26737-26740, (2001).

32 Prasad, V. et al. Haploinsufficiency of $\langle$ em $>$ Atp2a2 $</$ em $>$, Encoding the Sarco(endo)plasmic Reticulum Ca $<$ sup $>2+</$ sup $>$-ATPase Isoform 2 Ca $<$ sup $>2+</$ sup $>$ Pump, Predisposes Mice to Squamous Cell Tumors via a Novel Mode of Cancer Susceptibility. Cancer Research 65, 86558661, (2005).

33 Stairs, D. B. et al. Deletion of p120-catenin results in a tumor microenvironment with inflammation and cancer that establishes it as a tumor suppressor gene. Cancer cell 19, 470483, (2011).

34 Wu, Y. et al. Therapeutic antibody targeting of individual Notch receptors. Nature 464, 10521057, (2010).

35 Wang, L. et al. Unbalanced YAP-SOX9 circuit drives stemness and malignant progression in esophageal squamous cell carcinoma. Oncogene 38, 2042-2055, (2019).

36 Chen, L. et al. CDK14 involvement in proliferation migration and invasion of esophageal cancer. Annals of translational medicine 7, 681, (2019).

37 Fung, T. M. et al. Neuropilin-2 promotes tumourigenicity and metastasis in oesophageal squamous cell carcinoma through ERK-MAPK-ETV4-MMP-E-cadherin deregulation. The Journal of pathology 239, 309-319, (2016).

38 Lioni, M. et al. Dysregulation of claudin-7 leads to loss of E-cadherin expression and the increased invasion of esophageal squamous cell carcinoma cells. The American journal of pathology 170, 709-721, (2007).

39 Usami, Y. et al. Reduced expression of claudin-7 correlates with invasion and metastasis in squamous cell carcinoma of the esophagus. Hum Pathol 37, 569-577, (2006).

40 Jones, D. et al. cgpCaVEManWrapper: Simple Execution of CaVEMan in Order to Detect Somatic Single Nucleotide Variants in NGS Data. Current Protocols in Bioinformatics 56, 15.10.11-15.10.18, (2016).

41 Raine, K. M. et al. cgpPindel: Identifying Somatically Acquired Insertion and Deletion Events from Paired End Sequencing. Current protocols in bioinformatics 52, 15.17.11-15.17.12 (2015).

42 Gerstung, M., Papaemmanuil, E. \& Campbell, P. J. Subclonal variant calling with multiple samples and prior knowledge. Bioinformatics 30, 1198-1204, (2014).

43 Kemp, R. et al. Elimination of background recombination: somatic induction of Cre by combined transcriptional regulation and hormone binding affinity. Nucleic Acids Res 32, e92, (2004). 
bioRxiv preprint doi: https://doi.org/10.1101/2021.06.18.448956; this version posted June 18, 2021. The copyright holder for this preprint (which was not certified by peer review) is the author/funder, who has granted bioRxiv a license to display the preprint in perpetuity. It is made available under aCC-BY 4.0 International license.

44 Movahedan, A. et al. Loss of Notch1 disrupts the barrier repair in the corneal epithelium. PLoS One 8, e69113, (2013).

45 Anders, S., Pyl, P. T. \& Huber, W. HTSeq--a Python framework to work with high-throughput sequencing data. Bioinformatics 31, 166-169, (2015).

46 Dobin, A. et al. STAR: ultrafast universal RNA-seq aligner. Bioinformatics 29, 15-21, (2013).

47 Love, M. I., Huber, W. \& Anders, S. Moderated estimation of fold change and dispersion for RNA-seq data with DESeq2. Genome biology 15, 550, (2014).

48 Yu, G., Wang, L. G., Han, Y. \& He, Q. Y. clusterProfiler: an R package for comparing biological themes among gene clusters. Omics 16, 284-287, (2012). 


\section{Methods}

\section{Human samples collection and processing}

Collection. Esophageal tissue was obtained from deceased organ donors from whom organs were being retrieved for transplantation. Informed consent for the use of tissue was obtained from the donor's family (REC reference: 15/EE/0152 NRES Committee East of England - Cambridge South). A full thickness segment of mid-esophagus was excised within 60 minutes of circulatory arrest and preserved in University of Wisconsin (UW) organ preservation solution (Belzer UW ${ }^{\circledR}$ Cold Storage Solution, Bridge to Life, USA) until processing. Tissue was flash frozen in tissue freezing medium (Leica catalog no. 14020108926).

Sampling for sequencing. For sequencing $20 \mu \mathrm{m}$ thick cryosections were fixed with $4 \%$ paraformaldehyde for $10 \mathrm{~min}$. Sections were stained for NOTCH1 (Cell Signaling catalog no. 3608) using the Rabbit specific HRP/DAB (ABC) Detection IHC Kit (Abcam Plc catalog no. ab64261) and counterstained with Haematoxylin. 250 to $1000 \mu \mathrm{m}$ lengths of epithelium sections were microdissected from 4 to 6 successive serial sections and DNA extracted using a QIAMP DNA microkit (Qiagen) by digesting overnight and following manufacturer's instructions. Flash frozen esophageal muscle DNA was used as the germline control and DNA extracted as for the epithelial samples.

Targeted sequencing. Samples were multiplexed and sequenced on an Illumina HiSeq 2000 sequencer using paired-end 75-base pair (bp) reads. Agilent SureSelect custom bait capture kits were used (Supplementary table 1). Details on DNA inputs, sequencing alignment, coverage metrics and pipelines used for mutation calling and copy number analysis are given in the Supplementary Note. Total number of protein altering mutations among the 60 sequenced samples mentioned in the main text are from CaVEMan and cgpPindel variant calling algorithms ${ }^{40,41}$. NOTCH1 mutations shown in Fig.1c were generated from these two algorithms with the addition of calls from ShearwaterML algorithm ${ }^{1,5,42}$ which detects mutations with low variant allele frequency, see Supplementary table 2c.

Immunostaining and analysis of contiguous segments. For in situ quantifications, $10 \mu \mathrm{m}$ thick serial cryosections were fixed with $4 \%$ paraformaldehyde for $10 \mathrm{~min}$. For NOTCH1 and NICD1 detection and quantification Fig. 1d-e), three successive sections were stained respectively for NOTCH1/KRT4/ITGA6/DNA (1), NICD1/KRT14/DNA (2) and NOTCH1/KRT14/ITGA6/DNA (3) and imaged in their entirety. Corresponding areas in each section were identified based on tissue structure, distances and staining. Contiguous areas were analyzed for presence or absence of NOTCH1 or nuclear NICD1 staining and their length along the section measured using Volocity 6 software (Perkin Elmer). For morphological analysis of NICD1 and positive negative areas (Extended data Fig.1 f-h), sections were stained for NICD1/KRT14/DNA or NICD1/Ki67/DNA and epithelial thickness measured and cell counting performed using Volocity 6 software (Perkin Elmer). Cell density was measured by counting the number of nuclei identified across the width of the epithelium on high resolution images of cryosections stained with DAPI and keratinocyte markers such as KRT4 and ITGA6 and dividing this number by the measured surface area. 


\section{Animals}

All experiments were conducted according to the UK Home Office Project Licenses P14FED054 and PF4639B40. Male and female adult mice were used for in vivo experiments. Animals were housed in individually ventilated cages and fed on standard chow and maintained at SPOF health status. B6.129X1-Notch $1^{\text {tm2Rko/GridJ }}$ mice were purchased from the Jackson Laboratory and crossed with Rosa26 floxedYFP and AhCre ${ }^{E R T}$ to generate YFPCreNotch1 triple mutant mice (Extended data Fig. 2b-d) $13,17,43$. C57BL/6J wild-type mice were also used as indicated.

\section{qPCR Recombination assay}

Genomic DNA from esophageal epithelium whole mount tissue was extracted using QIAamp DNA Micro Kit (Qiagen, catalog no. 56304). Specific primer sets were designed to analyze excision of the floxed exon1 of Notch1 by Cre recombinase (Extended data Fig. 3c). Primer set A allows intragenic normalization using the non-floxed Notch1 exon 3: Forward: GCCTGACACCTCTGGACAAC; Reverse: GCGGCACTTGTACTCTGTGA. Primer set B measures the disappearance floxed exon 1 with recombination: Forward: AGGAAAGAGGGCATCAGAGG; Reverse: GGACGTTGACGCACACCA. Quantitative PCR on genomic DNA was carried out using specific primers and SYBR Green master mix (Thermo Fisher Scientific, catalog no. 4309155) according to the manufacturer's instructions in a StepOnePlus ${ }^{\text {TM }}$ Real-Time PCR System (Thermo Fisher Scientific, catalog no. 4376600). Relative q-PCR expression was calculated using delta-delta $\mathrm{Ct}$ method, a wild type reference sample was used within the same assay. Validation of the linearity of the recombination assay was performed against a standard curve reproducing different recombination rates with Exon1/Exon3 ratio of 1, 0.75, 0.5, 0.25 and 0 . The Standard curve was made using diluted genomic DNA from the esophagus of highly induced and fully recombined Notch $1 \%$ mice (as verified by $\mathrm{QPCR}$, staining and protein assay) and from Notch1 wild type tissue. Full recombination of the esophageal epithelium will reduce the Exon1/Exon3 ratio to zero in induced Notch $1^{-/}$mice and to 0.5 in induced Notch $1^{+-}$mice (Extended data Fig. 3e, Extended data Fig.6a).

\section{RT-qPCR assay}

RNA extractions were performed on mouse esophageal tissue as described in the RNA sequencing method section. Total RNA was measured using Qubit ${ }^{\mathrm{TM}}$ RNA BR Assay Kit (Thermo Fishcer Scientific, catalog no. Q10211). cDNA synthesis of 500ng total RNA was performed using QuantiTect Reverse Transcription Kit (Qiagen, catalog no. 205313). RT-qPCR was performed with Taqman Fast Advanced Master Mix (Thermo Fisher Scientific, catalog no. 4444557) on StepOnePlus ${ }^{\mathrm{TM}}$ Real-Time PCR System (Thermo Fisher Scientific, catalog no. 4376600) and analyzed using StepOne Software v2.3. Relative q-PCR expression to Gapdh housekeeping gene was calculated using delta-delta Ct method. The specific Taqman primers used for quantification were the following: Gapdh: Mm99999915_g1; Notch1: Mm00627185_m1; Gli2: Mm01293117_m1; lgfbp3: Mm01187817_m1; Adam8: Mm00545745_m1; Tgm2: Mm00436979_m1; Sox9: Mm00448840_m1.

\section{Immune Capillary Electrophoresis}


RLT Plus lysates with Complete Protease Inhibitor (Roche, catalog no. 11836170001) homogenized as described in the 'RNA sequencing' section were passed through the RNA binding column from the AllPrep DNA/RNA Mini kit (Qiagen) and the flow through was collected for protein precipitation. For precipitation, 9 volumes of ice cold pure Ethanol were mixed to the lysates before storage overnight at -80 degrees. Precipitates were spun $30 \mathrm{~min}$ at $20,000 \mathrm{~g}$ at 4 degrees, pellets were dried and solubilized progressively with $5 \%$ Sodium dodecyl sulfate in $100 \mathrm{mM}$ TEAB solution (Sigma-Aldrich, catalog no. T7408). Total protein quantification was performed using Pierce ${ }^{\mathrm{TM}}$ BCA Protein Assay Kit (Thermo Fisher Scientific, catalog no. 10678484)). Immune capillary electrophoresis was performed using Wes Simple ${ }^{\mathrm{TM}}$ (ProteinSimple) following manufacturer's instructions and analyzed using Compass for SW version 4.1.0. Primary antibodies were the following: anti-NOTCH1 targeting $\mathrm{C}$ terminus of the protein (Cell signaling, catalog no. 3608); anti-NOTCH2 targeting $\mathrm{C}$ terminus of the protein (Cell signaling, catalog no. 5732); alpha-TUBULIN (Cell signaling, catalog no. 2125).

\section{Whole-mount sample preparation of mouse esophagus}

Mouse esophagus was cut and opened longitudinally before removing the muscle layer with forceps. For the clonal lineage tracing experiments, the tissue was incubated for 15 minutes in Dispase I (Roche catalog no. 04942086001) diluted at $1 \mathrm{mg} / \mathrm{ml}$ in PBS before separating the epithelium with fine forceps. For all the other immunostaining experiments, tissue was incubated for $2 \mathrm{~h} 15$ to $3 \mathrm{~h}$ in 5 mM EDTA at $37{ }^{\circ} \mathrm{C}$ before gentle peeling of the epithelium. The epithelium was flattened and fixed in $4 \%$ paraformaldehyde for $1 \mathrm{~h} 15$ at room temperature under agitation. Tissues were then washed in PBS and stored at $4{ }^{\circ} \mathrm{C}$.

\section{Whole mount immunostaining}

Whole mount tissues were blocked an hour in staining buffer (0.5\% BSA, 0.25\% fish skin gelatin, $0.5 \%$ Triton X-100, 10\% donkey serum in PHEM). Samples were incubated with primary antibodies (Supplementary Table 8) in staining buffer overnight at room temperature, followed by three washes of $30 \mathrm{~min}$ with $0.2 \%$ Tween-20 in PHEM. Samples were incubated with secondary antibodies (Supplementary Table 8) in staining buffer for $3 \mathrm{~h}$ at room temperature and washed. Finally, tissues were incubated overnight at room temperature with $1 \mu \mathrm{g} \cdot \mathrm{ml}^{-1}$ DAPI or $0.5 \mu \mathrm{M}$ Sytox ${ }^{\mathrm{TM}}$ Blue solution (Biolegend, catalog no. 425305, see section on 'preparation of mouse samples for sequencing') to counterstain cell nuclei and mounted using Vectashield mounting media (Vector Laboratories, catalog no. $\mathrm{H}-1000)$.

\section{Cryosections}

Esophageal tissue was flash frozen in tissue freezing medium (Leica catalog no. 14020108926). $10 \mu \mathrm{m}$ transversal sections were fixed with $4 \%$ paraformaldehyde for $10 \mathrm{~min}$, blocked in staining buffer $(0.5 \%$ BSA, $0.25 \%$ fish skin gelatin, $0.5 \%$ Triton X-100, 10\% donkey serum in PHEM) and stained with the respective primary and secondary antibodies for 3 hours to overnight at room temperature (Supplementary Table 8). Samples were washed with PHEM buffer between incubations. Prior to NICD1 staining, sections were incubated 20 minutes in 50mM Glycine/PBS solution. Finally, tissues were incubated overnight at room temperature with $1 \mu \mathrm{g} \cdot \mathrm{ml}^{-1}$ DAPI or $0.5 \mu \mathrm{M}$ Sytox ${ }^{\mathrm{TM}}$ Blue solution (Biolegend, catalog no. 425305) to counterstain cell nuclei and mounted using Vectashield mounting media (Vector Laboratories, catalog no. H-1000). 


\section{Histology}

Hematoxylin and eosin staining was either performed on $10 \mu \mathrm{m}$ cryosectioned tissue processed as described above or on $5 \mu \mathrm{m}$ paraffin embedded tissue sections. Prior paraffin embedding, esophageal tissue was collected and fixed in $4 \%$ paraformaldehyde for at least 2 hours before undergoing progressive dehydration in Tissue-Tek VIP ${ }^{\circledR} 6$ Al tissue processor (Sakura). Slides were then scanned at objective 20x using NanoZoomer S60 Digital slide scanner (Hamamatsu).

\section{Confocal microscopy}

Immunofluorescence images were acquired on a Leica TCS SP8 confocal microscope using $\times 10, \times 20$ or $\times 40$ objectives. Typical settings for acquisition were optimal pinhole, line average $3-4$, and scan speed $400-600 \mathrm{~Hz}$ and a resolution of $1024 \times 1024$ pixels. Visualization and image analysis were performed using Volocity 6 Image Analysis software (PerkinElmer).

\section{Lineage tracing using a YFP reporter}

To genetically label wild type and Notch1 mutant clones in the same tissue, we crossed floxed Notch1 mice with floxed Rosa26 ${ }^{\text {EYFP }}$ animals carrying a conditional fluorescent YFP reporter at the Rosa26 locus and the inducible $A h C r e^{E R T}$ mice ${ }^{13,17}$. Pharmaceutical treatment with $\beta$-naphthoflavone (BNF, MP Biomedicals, catalog no.156738) and tamoxifen (TAM, SigmaAldrich, catalog no. N3633) allows the expression and nuclear translocation of the Cre protein and the subsequent recombination of the floxed Notch1 resulting in the excision of its first exon and/or Rosa26 alleles resulting in the expression of the fluorescent reporter YFP. Titration of the dosage of BNF/TAM was performed to allow recombination of in a small proportion of the esophageal basal cells, called clonal level. This allows the recombined cells to expand over time and generate clones without fusion. Excision of the Notch1 allele and expression of the YFP reporter at the Rosa26 locus can occur in the cells either in combination or separately, resulting in four different population of cells, Notch1 mutant or not and expressing YFP or not (Extended data Fig. 2b, c). Mice between 10-16 weeks of age were injected intraperitoneally (i.p.) with BNF (80 mg. $\left.\mathrm{kg}^{-1}\right)$ and TAM $(0.125 \mathrm{mg})$ and collected at different time points. Staining and processing of the whole mount tissue allowed us to track the clones based on YFP and NOTCH1 expression (Extended data Fig. 3). YFP expressing clones were imaged with confocal microscopy at 40x objective. In induced Notch $1^{\text {floxflox }}$ tissue, YFP+ clones expressing NOTCH1 were categorized as wild type, while the clones without detectable expression of NOTCH1 were classified as Notch $1^{-}$.

In order to distinguish the wild type and Notch1+/- clonal populations in induced Notch $1^{+/ f l o x e d}$ tissue, we set up a method based on measurement of NOTCH1 staining. For each clone, the mean intensity of NOTCH1 immunofluorescence was recorded in the basal cells expressing the YFP reporter, normalized to the mean intensity of the corresponding DNA stain in each image. We analyzed the NOTCH1 staining intensity distribution in 3 wild type mice in $n=126 \mathrm{YFP}+$ clones and used this as a reference (Extended data Fig. $3 \mathrm{~h}$ ). The distribution of the relative intensities could be approximated with a normal curve ( $p=0.25$, Shapiro-Wilks test) with mean 1.02 and standard deviation 0.15 , fit with Python package Scipy v1.5.2. The distribution of relative NOTCH1 staining intensity of the $n=172$ YFP

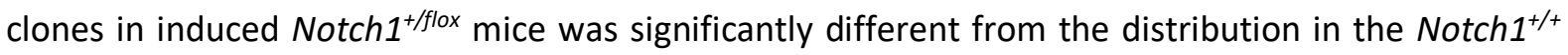
mice ( $p=5 \mathrm{e}^{-27}$, two-tailed Kolmogorov-Smirnov test). To confirm that the clones in induced Notch $1^{+f f l o x}$ mice were a mixture of Notch1 wild type and Notch $1^{+/-}$clones, we fit a Gaussian Mixture model with 
two components to the Notch $1^{+/ f l o x}$ relative intensity distribution using the Python package Scikit-learn v0.23.2. The resultant fit consisted of a normal distribution highly similar to that fit to the clones from the Notch $1^{+/+}$mice (mean=1.0, sd=0.15), and a normal distribution with half the intensity of the Notch1 wild type clones (mean $=0.53, \mathrm{sd}=0.15$ ). We chose to split the induced Notch $1^{+/}$clones using a relative intensity threshold of 0.75 . This threshold is slightly lower than the mid-point of the two distributions, and was selected so that very few of the wild type clones $(<5 \%)$ would be categorized as Notch $1^{+<}$. The higher intensity clones were assumed to be Notch1 wild type $(+/+$ in $+/$ flox $)$ and had a similar distribution of staining intensity to those in the Notch $1^{+/+}$mice $(p=0.38$, two-tailed KolmogorovSmirnov test). The remaining clones were categorized as Notch1 ${ }^{+/-}$(Extended data Fig. 3e). These methods were used at each time point. Number of basal and suprabasal cells in each clone was counted using Volocity 6 Image Analysis software (PerkinElmer).

Clones with no basal cells in the induced mutant mice could not be categorized with this method but represented only $\sim 1 \%$ of the clones in the dataset. Fusion of expanding clones prevented quantification of clone size beyond 4 weeks in induced Notch $1^{\text {flox/flox }}$ esophagus and beyond 13 weeks in induced Notch $1^{+/ \text {flox }}$ tissue.

\section{EDU lineage tracing}

EdU (5-ethynyl-2'-deoxyuridine) incorporates during replication in the proliferating cells located in the basal layer of the esophageal epithelium (Extended data Fig. 2a). EdU i.p. injection at $10 \mu \mathrm{g}$ was performed either at 1 hour or $48 \mathrm{~h}$ before tissue collection. Tissue was processed and EdU was detected in whole mount using Click-iT EdU imaging kit (Life Technologies, catalog no. C10338 or C10340). Images were acquired by confocal microscopy and analyzed using Volocity 6 software.

Performed 1 hour before collection, the assay was used to quantify the proliferation rate in the esophageal epithelium. The proportion of EdU+ basal cells: total basal cells was recorded as a measure of proliferation. Performed $48 \mathrm{~h}$ before collection, this assay was used to quantify differentiation. Indeed, the EdU labelled cells generate daughter cells that can either stay in the basal layer or differentiate and leave the basal layer. The proportion of EdU positive suprabasal cells relative to total $\mathrm{EdU}+$ cells was recorded as a measure of cell stratification. In the clonal induction experiment, the wild type neighbor cells of the mutant clones called $+/+$ edge were identified as the first row of cells adjacent to the YFP expressing cells while the distant cells called $+/+$ distant were randomly situated further away from the clone (Fig.2 g-n).

\section{Aging experiments}

YFPCreNotch1 mice between 10-16 weeks of age were injected intraperitoneally (ip) with BNF at 80 $\mathrm{mg} . \mathrm{kg}^{-1}$ and TAM at $1 \mathrm{mg}$ for Notch $1^{+/+}$and Notch $1^{+/ f l o x-}$ and at $0.25 \mathrm{mg}$ for Notch $1^{\text {flox flox }}$ and collected at different time points after induction and up to 78 week-old. (Fig. 3c, Fig.5, Extended data Fig.5). Notch $1^{+/+}$or non-induced mice were used as wild type controls as indicated. A lower dose of Tamoxifen was used for the Notch $1^{\text {floxfflox }}$ mice in order to minimize the recombination of the Notch1 allele in the corneal epithelium, possibly leading to corneal opacification and keratinization ${ }^{44}$.

\section{Projected NOTCH1 stained area quantification}


To quantify the percentage of NOTCH1 positive or negative area in the entire esophageal epithelium or the projected surface of NOTCH1 negative clones (Fig. 3b, Fig. 6e, Extended data Fig. 8i), whole mount esophageal epithelia were prepared and stained for NOTCH1 and counterstained with DAPI or Sytox ${ }^{\mathrm{TM}}$ Blue as described in the dedicated sections. Tissues were imaged entirely using high precision motorized stage coupled to a Leica TCS SP8 confocal microscope to obtain contiguous 3D images of all epithelial layers and merged using the mosaic function of the Leica software. Typical settings for acquisition of multiple $z$ stacks were optimized $2.41 \mu \mathrm{m} z$ step size, zoom $\times 1$, optimal pinhole, line average 4 , scan speed $400-600 \mathrm{~Hz}$ and a resolution of $1024 \times 1024$ pixels using an $\times 10 \mathrm{HC}$ PL Apo CS Dry objective with a 0.4 numerical aperture. Images were processed using Volocity 6 software. To measure their projected surface area, images were opened using the 'extended focus' visualization mode on the Volocity software. Regions of interest were defined with ROI tool allowing surface area measurement. NOTCH1 staining was automatically detected based on defined intensity and minimum object size.

\section{Detection of spontaneous mutant clones and ex vivo Notch1 mutant functional assay in aging Notch1 ${ }^{+/-}$tissue}

Esophageal tissue from induced YFPCreNotch $1^{+/}$mice aged to 54-78 week-old was incubated for $2 \mathrm{~h} 30$ in $5 \mathrm{mM}$ EDTA solution at $37^{\circ} \mathrm{C}$ before peeling. Whole mount esophageal epithelium was stained for NOTCH1/YFP and Sytox ${ }^{\mathrm{TM}}$ Blue and imaged. In the aged YFPCreNotch $1^{+-}$tissues, the detection of large and ovoid areas negative for NOTCH1 staining allowed the identification of putative spontaneous NOTCH1 negative clones (Fig. 3b, e). To identify putative clones expressing NOTCH1, we used the combination of NOTCH1 and YFP staining. In highly induced heterozygous tissue, YFP staining normally appear as a patchwork of small YFP+ clones. Putative clones appeared as large and ovoid areas devoid or fully stained with YFP (Fig.3e). Sequencing confirmed that this method allowed the identification of large spontaneous clones carrying Notch1 mutations on the non-recombined allele (Fig. 3e-g). EDTA treatment triggers the cleavage of NICD1, followed by nuclear translocation without ligand activation 20. We used this assay to test the functionality of the cleavage of the spontaneous NOTCH1 positive clones identified (Extended data Fig. 5e-j).

\section{Carcinogen treatment}

Mice were induced with BNF/TAM at a dose that allowed full coverage of the tissue with the mutant Notch1 heterozygous or homozygous cells within three months. YFPCreNotch $1^{+/ f l o x}$ mice were injected intraperitoneally (i.p.) on two consecutive days with BNF at $80 \mathrm{mg} \cdot \mathrm{kg}^{-1}$ and TAM at $1 \mathrm{mg}$ for and YFPCreNotch $1^{\text {flox/flox }}$ were injected once with BNF at $80 \mathrm{mg} \mathrm{kg}^{-1}$ and TAM at $0.25 \mathrm{mg}$. Non-induced YFPCreNotch1 mice were used as controls. Then, in order the generate mutations and tumor formation in the esophageal epithelium, mice were treated with DEN (Sigma, catalog no. N0756) in sweetened drinking water $(40 \mathrm{mg} / \mathrm{L}$ ) for 24 h, 3 days a week (Monday, Wednesday and Friday) for 8 weeks ${ }^{16,19}$. Sorafenib (LC Chemicals, catalog no. S8502) was then administered at $50 \mathrm{mg} / \mathrm{kg}$ (5 microliters of $10 \mathrm{mg} / \mathrm{ml}$ solution per $\mathrm{g}$ bodyweight) by i.p. injection on alternate days during 6 weeks, for a total of 21 doses ${ }^{29}$.

Mice were aged for 28 weeks after the last dose of Sorafenib and esophageal tissue was collected and opened longitudinally. Macroscopic images of unpeeled tissue were obtained under Leica M80 zoom stereomicroscope with Leica Plan 1.0x Objective M-Series 10450167 coupled with Leica DFC295 Camera (Leica microsystems). Macroscopic tumors were punched and flash frozen in tissue freezing 
medium (Leica catalog no. 14020108926) for further processing. Esophageal tissue was peeled for 3 hours in $5 \mathrm{mM}$ EDTA, fixed, processed through whole mount immunostaining for the Keratin 6a stress marker and DNA staining before confocal imaging. Detailed analysis of macroscopic and microscopic images was performed to quantify the projected areas of the lumps using ROI tool in Volocity 6 software.

\section{Antibody treatment validation}

Antibody validation was performed in two steps. For step 1, C57BL/6J were injected intraperitoneally at high dose with rat anti-Notch1 NRR hybridoma Clone 1E3.19.1 (anti-NRR1.1E3, Genentech) at $25 \mathrm{mg} \cdot \mathrm{kg}^{-1}$ ( $\mathrm{n}=3$ mice per group). Anti-NRR1.1E3 is distinct from the anti-NRR1 from Wu et al. ${ }^{34}$. This rat monoclonal antibody has a lower affinity for mouse anti-NRR and is better tolerated. Antibody Ragweed:9652 10D9.W.STABLE mlgG2a (CTRL, Genentech) was used as control at $25 \mathrm{mg} / \mathrm{kg}^{-1} .3$ days later, esophageal tissue was collected and processed for RT-qPCR assay and protein quantification as described in the 'RT-qPCR' and 'Immune Capillary Electrophoresis assay' (ICE) sections. At the protein level, cleaved transmembrane/intracellular regions (NTM + NICD) of NOTCH1 and NOTCH2 were quantified by ICE. The assay confirmed the specificity of the antibodies for NOTCH1 neutralization (Extended data Fig.8a-c). Tissue was also sectioned and stained for NICD1, confirming the absence of active NOTCH1 in basal keratinocytes with the anti-NRR1.1E3 treatment (Extended data Fig.8d). At the transcriptional level, RT-qPCR for Notch1 loss of functions markers Igfbp3, Tgm2, Gli2, Adam8 and Sox9 were performed (Extended data Fig.8e). For step 2, treatment doses were titrated to allow efficiency and safety of the treatment over an extended period of time. YFPCreNotch $1^{\text {floxfflox }}$ mice were induced at clonal level, and starting a week later, were treated once a week with the antibodies for 3 weeks and tissue was collected, peeled and whole mount stained for NOTCH1. As expansion of clones is subject to the fitness of the neighbor cells ${ }^{19}$, a total NOTCH1 blockade would be expected to neutralize the competitive advantage of $\mathrm{Notch}^{1}$ clones over wild type cells, reducing clonal expansion. NOTCH1 negative staining areas were therefore measured as to determine the efficacy of the antibody in inhibiting NOTCH1 in esophageal epithelium (Extended data Fig. 8f-i). Anti-NRR1.1E3 at $10 \mathrm{mg} . \mathrm{kg}-1$ offered the strongest reduction in NOTCH1 negative clone size showing the efficacy of the treatment. The health and weight of the mice was assessed through the treatment. $n=4$ mice injected with Ragweed controls at $10 \mathrm{mg} / \mathrm{kg} ; \mathrm{n}=3$ mice treatedNRR1.1E3 at $10 \mathrm{mg} / \mathrm{kg} ; \mathrm{n}=2$ mice given NRR1.1E3 10mg/kg loading dose (week 1: $10 \mathrm{mg} / \mathrm{kg}$, week 2: 7.5, week $3: 5 \mathrm{mg} / \mathrm{kg}$ ); $\mathrm{n}=1$ mouse given NRR1.1E3 5mg/kg; and n=1 mouse given NRR1.1E3 5mg/kg loading (week1: $5 \mathrm{mg} / \mathrm{kg}$, week 2: $4 \mathrm{mg} / \mathrm{kg}$, week $3: 3 \mathrm{mg} / \mathrm{kg}$ ). Antibody NRR1.1E3 did not lead to any weight loss or observable adverse effects.

\section{Immunotherapeutic treatment}

YFPCreNotch $1^{+/+}$mice were first treated with DEN and Sorafenib and aged for 9 weeks to allow the tumors to start growing before starting a treatment with anti-NRR1.1E3 at 10mg.kg-1 or with Ragweed control at 10mg.kg-1 ( $n=4$ mice per group), once a week for 6 weeks (Fig. 7i-k). One week after the last dosage, tissue was collected and processed for macroscopic and microscopic quantification of the projected areas of the tumors using Volocity 6 software as described in 'carcinogen treatment' section

\section{Cell density in mouse esophagus}

For aged mouse tissue (Fig. 5c), density of the basal cells was measured on whole mount stained tissue, imaged at 40x objective using Leica TCD SP8 confocal microscope (see 'confocal microscopy'). 
DAPI+ or Sytox ${ }^{\mathrm{TM}}$ Blue + basal nuclei were quantified per area in 3 to 6 random positions of the tissue, $\mathrm{n}=4$ mice per genotype analyzed.

\section{Cell counting and epithelial thickness in mouse tissue cryosections}

Esophageal epithelial thickness was quantified in transversal cryosections stained with Hematoxylin and eosin (H\&E) with NanoZoomer Digital Pathology software (NDP.view2, Hamamatsu). Measurements were performed at 18 to 23 positions in the tissue and averaged for each mouse $(n=3)$. For cell counting in cryosections, stained sections were imaged using Leica TCD SP8 confocal microscope at 40x objective as described in 'confocal microscopy' and analyzed with Volocity 6 software (Perkin Elmer). For counting Ki67 positive basal cells in the aged tissue (Fig.5d), tissue was stained for Ki67 \& DNA and analysis were performed at three different positions per animal and averaged for each mouse (n=3-4). For Ki67 counting in the tumors (Extended data Fig.7f), tissue cryosections were stained for Ki67, KRT14 and DNA to identify keratinocytes in the tumor mass ( $\mathrm{N}=8$ 9 tumors from $n=5$ Notch $^{+/+}$and 6 Notch $1^{-1}$ mice respectively). For NICD1 counting, sections of tumors and adjacent normal tissue were stained for NICD1, KRT14 and DNA. Proportion of KRT14+ keratinocytes expressing nuclear NICD1 inside the tumor mass and in the adjacent normal epithelium was quantified ( $n=10$ tumors from 6 mice).

\section{Projected E-cadherin intensity quantification}

Esophageal sections carrying tumors and adjacent normal tissue were stained for E-cadherin, Keratin 14, counterstained with DAPI (Fig. 7f-h) and imaged at high resolution using Leica TCD SP8 confocal microscope as described in the previous sections. To measure the intensity of the staining on projected areas, images were opened using the 'extended focus' visualization mode on the Volocity 6 software. Regions of interest (adjacent normal epithelium and keratinocytes populated tumor mass) were defined with ROI tool and mean intensity within areas were quantified. Mean intensity of Ecadherin was normalized on the mean intensity of DAPI for each ROI. Analysis compared measures in the tumors to the measures in the adjacent normal tissue ( 8 tumors from 5 wild type mice; 8 tumors from 6 Notch $1 \%$ mice). We verified that E-cadherin staining was not affected in the normal tissue of DEN/SOR treated Notch1\% mice compared to wild type mice using this method on tissues embedded in the same OCT bloc and stained together on the same slide ( $n=3$ mice).

\section{RNA sequencing}

YFPCreNotch $1^{\text {flox/flox }}$ and YFPCreNotch $1^{\text {tflox }}$ mice between 10-16 weeks of age were injected intraperitoneally (i.p.) on two consecutive days with BNF at $80 \mathrm{mg} . \mathrm{kg}-1$ and TAM at $1 \mathrm{mg}$ and aged for 8 weeks before collection (Fig. 4a). The dose was set in allow Notch $1^{+/}$or by Notch $1^{\%}$ cells to fully occupy the tissue by two months post induction, as measured by qPCR recombination assay (Extended data Fig.3e), qPCR assay and protein assay (Fig.2b,c). Uninduced littermates were used as wild type control. Esophageal tissues were incubated for 15 minutes in Dispase I (Roche catalog no. 04942086001) diluted at $1 \mathrm{mg} / \mathrm{ml}$ in PBS before separating the epithelium with fine forceps. Peeled epithelium were lysed in RLT Plus buffer using MagNA Lyser Green Beads (Roche, catalog no. 03358941001) and FastPrep 24 homogenizer (MP Biomedicals, catalog no.116004500). Lysates were 
then processed for RNA and DNA extraction using AllPrep DNA/RNA Mini kit (Qiagen), with Complete Protease Inhibitor (Roche, catalog no. 11836170001) added instead of the proteinase K step, allowing protein extraction as described in the Immune Capillary Electrophoresis section. Total RNA was measured using Qubit ${ }^{\text {TM }}$ RNA BR Assay Kit (Thermo Fisher Scientific, catalog no. Q10211).

For RNA-seq, libraries were prepared in an automated fashion using an Agilent Bravo robot with aKAPA Standard mRNA-Seq Kit (KAPA BIOSYSTEMS). In-house sequencing adaptors were ligated to 100-300 bp fragments of input dsDNA. Samples were then subjected to 10 PCR cycles using sanger_168 tag set of primers and paired-end sequencing was performed on Illumina HiSeq 2500 with $75 \mathrm{bp}$ read length. Reads were mapped using STAR 2.5.3a, the alignment files were sorted and duplicate-marked using Biobambam2 2.0.54, and the read summarization performed by the htseqcount script from version 0.6.1p1 of the HTSeq framework ${ }^{45,46}$.Differential gene expression was analyzed using the DESeq2 $\mathrm{R}$ package ${ }^{47}$ and the downstream pathway analysis and visualization using $\mathrm{R}$ (https://www.R-project.org/) and the packages Pheatmap (https://cran.rproject.org/package=pheatmap), RColorBrewer (https://cran.r-project.org/package=RColorBrewer), clusterProfiler 48 and org.Mm.eg.db (https://bioconductor.org/packages/org.Mm.eg.db/). Background noise was filtered out from the dataset using the following rule: genes with an eigenvector value of $>0.05$ or $<-0.05$ for both PC1 and PC2 among wild type control samples were removed from the dataset (151 genes out of the initial 47,467 genes) for final analysis. Differentially-expressed genes (DEG) are hits reported by DESeq2 with adjusted $p$ value ( $p \_$adj) of less than 0.05 .

Heatmaps were generated from the ratio of TPM values of the treated sample over the average of the respective control samples. The volcano plots are plotted using the $\mathrm{R}$ package plotrix to enable gapped axis (https://cran.r-project.org/package=plotrix) using the output from DESeq2 as input.

\section{Single cell RNA sequencing}

YFPCreNotch $1^{\text {flox/flox }}$ mice were injected intraperitoneally (ip) with BNF at $80 \mathrm{mg} \cdot \mathrm{kg}^{-1}$ and TAM at $1 \mathrm{mg}$ and aged for 11 weeks. Uninduced littermates were used as wild type controls. Mouse esophagus was collected and placed immediately in PBS on ice. Esophagus was opened and cut into 4 equal pieces and placed in $1 \mathrm{ml}$ dispase $(2.4 \mathrm{U} / \mathrm{ml})$ at $37 \mathrm{C}$ for $10 \mathrm{mins}$. Epithelia was then dissected away from the underlying stroma and muscle. Resulting epithelia was minced very finely using a scalpel and the slurry placed in $1 \mathrm{ml}$ trypsin-EDTA for 30mins at 37C. After incubation each sample was placed in $9 \mathrm{ml}$ ice-cold 0.04\% BSA/PBS in a C-type GentleMacs tube (Miltenyi Biotec). Tissue was further dissociated using a GentleMACs dissociator with programs $A 1$ and two rounds of program B1 with the sample kept on ice between programs. Samples were briefly centrifuged and filtered through a 30um filter (Miltenyi Biotec). Samples were then centrifuged at $4^{\circ} \mathrm{C}$ for $10 \mathrm{mins}$ at $500 \mathrm{xg}$. The pellet was resuspended in ice-cold $0.04 \% \mathrm{BSA} / \mathrm{PBS}$ and centrifuged again a $4^{\circ} \mathrm{C}$ for $5 \mathrm{mins}$ at $300 \mathrm{xg}$. Pellet was resuspended in ice-cold $0.04 \%$ BSA/PBS. Cells were counted and sufficient volume was placed into a Chromium reaction to allow 5000 cell recovery. Single cells were collected using Chromium (10X) with version 3 chemistry following manufacturer's instructions with one mouse esophagus per inlet. cDNA was sequenced using HiSeq4000 (Illumina) with the following read length 28 bp read 1; 91 bp read 2; 8 bp index 1; 0 bp index 2 with a single inlet per lane. Details of analytic methods for sc-RNAseq are given in the Supplementary Note. 


\section{Mouse sample preparation for DNA sequencing}

Prior to sampling of the normal esophageal tissue, esophageal epithelium was processed for whole mount immunostaining and imaged with confocal microscopy as described in the corresponding sections. Tissue for sequencing was incubated for 1 hour with $0.5 \mu \mathrm{M}$ Sytox ${ }^{\mathrm{TM}}$ Blue solution (Biolegend, catalog no. 425305) to counterstain cell nuclei. For sequencing, DNA from the ears of the same mice was extracted with the same method and used as germline controls.

Punching. For subclonal analysis of stained areas of interest, stained and imaged tissue was flattened and punched under a Fluorescent Stereo Microscope Leica M165 FC (Leica) using $0.25 \mathrm{~mm}$ diameter punch (Stoelting, catalog no. 57391) (Fig. 3e, Extended data Fig.5). DNA from the microbiopsies was extracted using Arcturus ${ }^{\circledast}$ PicoPure $^{\circledR}$ DNA extraction kit (Applied Biosystems, catalog no. 11815-00) following the manufacturer's instructions).

Gridding. For analysis of the mutations in the healthy esophageal epithelium, the esophageal epithelium was flattened, mapped and cut into $2 \mathrm{~mm}^{2}$ contiguous biopsies (Fig. 6a-c, Extended data Fig. 8d-f). Samples were digested and DNA extracted using the QIAMP DNA microkit (Qiagen, catalog no. 56304) following the manufacturer's instructions.

Tumor sampling. Flash frozen tumors collected as described in the 'carcinogenesis' section were cut into serial $50 \mu \mathrm{m}$ cryosections and stained for KRT14, KRT6a and Sytox ${ }^{\mathrm{TM}}$ Blue (Fig. 6a-c, Extended data Fig. 7a,b). In order to separate the tumor mass from the surrounding normal tissue and the underlying submucosa, sections were micro-dissected under a Fluorescent Stereo Microscope Leica M165 FC (Leica) using a micro knife (FST, catalog no. 10317-14). Dissected tumor from serial sections were pooled and tumor DNA was extracted using the QIAMP DNA microkit (Qiagen, catalog no. 56304) following the manufacturer's instructions.

\section{Targeted Sequencing.}

Samples were multiplexed and sequenced on Illumina HiSeq 2000 sequencer using paired-end 75-base pair (bp) reads. Agilent SureSelect custom bait capture kits were used (Supplementary table 1). Details on the custom bait kit, DNA inputs, sequencing alignment and coverage metrics are given in the Supplementary Note.

Details of processing and analytic methods for mutation calling and copy number alteration analysis are given in the Supplementary Note.

\section{Modelling}

Stochastic simulations of clonal dynamics are explained in Supplementary Note.

\section{Statistical analysis}

Data are expressed as mean values \pm SEM unless otherwise stated. Statistical tests are indicated in figure legends. No statistical method was used to predetermine sample size. Data are expressed as mean values \pm SEM unless otherwise stated. Statistical tests are indicated in figure legends. No statistical method was used to predetermine sample size. Animals of the correct genotype were randomly assigned to experimental groups. The investigators were not blinded to allocation during experiments and outcome assessment. 


\section{Data availability}

Accession numbers for the datasets are as follow:-Targeted sequencing of Human esophageal epithelium microbiopsies (EGA): EGAD00001006969. -Targeted sequencing of aged Notch1 +/- mouse esophageal epithelium microbiopsies (ENA): ERP126992-Targeted sequencing of mouse normal esophageal epithelium 28 weeks after DEN SOR treatment (ENA): ERP126993 -Targeted sequencing of mouse esophageal tumours 28 weeks after DEN SOR treatment (ENA): ERP126994-Transcriptomic analysis of Notch1 mutant esophageal epithelium (ENA): ERP126995 -Single cell transcriptional analysis of Notch1 mutant esophageal epithelium (ENA): ERP126996.

\section{Code availability}

The codes developed in this study has been made publicly available and can be found at https://github.com/PHJonesGroup/Abby etal SI code

\section{Biological Resources}

Mouse strains are available from the Jax repository (https://www.jax.org), with the exception of the $A h c r e^{E R T}$ line which may be obtained by contacting the corresponding author. 


\section{Supplementary Note}

This supplementary note details the computational modelling used in this study to simulate clonal dynamics followed by a description of the DNA and sc-RNA sequencing approaches and pipelines used.

\section{Stochastic simulations of clonal dynamics}

\subsection{Previous models of mutant clonal dynamics in esophageal epithelium}

Murine esophageal epithelium (EE) consists of layers of keratinocyte cells and is maintained by the proliferation of cells in the basal layer (Extended data Fig. 2a). Each basal cell division can form a pair of cells that remain in the basal layer and go on to divide, a pair of cells that will differentiate and stratify into the suprabasal layers without dividing, or one cell of each fate. The outcome of each division is random, but in homeostasis the balance between dividing and differentiating cells is maintained (Extended data Fig. 2a) ${ }^{1}$ so that the overall number of proliferative cells remains constant. Due the random (stochastic) nature of the process, some cell lineages will be lost from the tissue and others will form large clones of the original cell ${ }^{1,2}$.

A genetic mutation in a basal cell may introduce a bias towards the production of more proliferating cells, leading to the non-neutral expansion of a mutant clone ${ }^{3,4}$. However, in normal EE, a clone cannot not expand indefinitely ${ }^{5,6}$. Mutant clones compete for the limited space in the tissue, and expanding clones revert towards more neutral growth once they encounter similarly fit clones ${ }^{6}$. Therefore, the fate of a cell division depends not only on the dividing cell, but also on the neighboring cells ${ }^{6}$. This spatial clonal competition in EE has been previously been modelled using stochastic simulations of cellular automata on fixed $2 \mathrm{D}$ grids ${ }^{6}$.

\subsubsection{Wright-Fisher simulations}

In this study, we wished to compare the growth of wild type $(+/+)$ clones, induced heterozygous Notch $1^{+-}$clones and induced homozygous Notch1 ${ }^{-/}$clones. We were looking for broad differences between growth rates, not for precise characterization of the clonal dynamics of each genotype. We therefore aimed to keep the model simple, with few free parameters, so that the results of parameter fitting for each genotype could easily be compared.

We used simulations based on a Wright-Fisher model ${ }^{7}$ constrained to a $2 \mathrm{D}$ grid ${ }^{8}$. Each cell, $C_{a, b}$, in the grid represents a cell in the basal layer of the EE. The subscripts denote the cell in location $a$ in the grid and generation $b$ of the simulation. We used a fixed size (500×500 cells) hexagonal grid with periodic boundary conditions.

Each cell has a fitness, $F_{a, b}$. In each step of the simulation, each cell $C_{a, b}$ in the new generation picks a parent cell from its immediate neighborhood $\mathcal{N}_{a}$ (the six adjacent cells plus the cell in the same grid position) in the previous generation (Extended data Fig. 4a). The chance of a cell being picked as the parent depends on its fitness relative to the other potential parent cells in the neighborhood,

$$
P\left(C_{a, b} \text { parent is } C_{a^{\prime}, b-1}\right)=\left\{\begin{array}{cc}
\frac{F_{a^{\prime}, b-1}}{\sum_{j \in \mathcal{N}_{a}} F_{j, b-1}} & a^{\prime} \in \mathcal{N}_{a} \\
0 & a^{\prime} \notin \mathcal{N}_{a}
\end{array}\right.
$$


A cell can produce multiple offspring in the next generation and the offspring cells inherit the fitness of their parent cells. In this way, a clone with high fitness can expand over multiple generations (Extended data Fig. 4a). At the start of a simulation, a small proportion of cells in the grid were randomly selected to be the induced mutant cells. In each simulation, a single fitness value was assigned to all the mutant cells (see section 1.4 below). The rest of the cells in the simulation were given a fitness of 1 . We tracked the sizes of the clones that grew from the mutant cells.

Division rate did not significantly differ between the wild type and Notch1 mutant tissue (Fig. 2g-j). We therefore fixed the division rate for all simulations at the division rate previously measured in the murine EE, 0.27 divisions per day ${ }^{1}$. The fitting results showed that this was appropriate for capturing the average growth of the wild type clones in this model (see section 1.4 below).

For each simulation, we had two parameters that were not fixed: the fitness of mutations and the induced proportion of cells at the start of the simulation. The induction proportion must be considered because as the clones expand they can collide and restrict each other's growth. In a highly induced tissue, fit mutant clones will have less room to expand and will be smaller at later time points than in a sparsely induced tissue. The other parameter, mutant fitness, is the key parameter we wish to compare between the genotypes. It represents how much of a growth advantage the mutant has compared to the surrounding non-mutant cells.

\subsection{Approximate Bayesian Computation}

To fit the parameters to the data we used Approximate Bayesian Computation $(A B C)^{9,10}$. In this fitting method, prior distributions for the parameters are defined. We used uniform distributions across wide intervals: mutant fitness between 0 and 50 , induction between $0 \%$ and $10 \%$ of cells. A parameter set is randomly drawn from the prior distributions, and a simulation run with those parameters. The simulation results are measured against the observed data using summary statistics. We used the Kolmogorov-Smirnov statistic ${ }^{11}$ to compare the simulated and experimentally observed basal clone size distributions. We used 100 simulated clones at each time point, and summed the KolmogorovSmirnov statistics calculated for each time point to get the total "distance" between the simulation and the data. If the distance between the simulation and the experimental data is less than a set threshold, those simulation parameters are accepted, otherwise they are rejected. Repeating this process many times with parameter sets randomly drawn from the prior distributions builds up a set of accepted parameters, which approximates the posterior parameter distribution ${ }^{10}$.

We used a version of $A B C$ based on sequential Monte Carlo sampling (ABC-SMC) ${ }^{12}$. This reduces the required number of simulations by running multiple generations of simulations, with new generations of parameters based on the successful parameters from the previous generation. The threshold distance for parameter acceptance is reduced with each generation so that the distribution of accepted parameter sets increasingly approaches the target posterior distribution ${ }^{12}$. We used the $A B C-S M C$ implementation from the python package PyABC ${ }^{13}$, and ran 15 generations using a population of 1000 particles (the required number of accepted parameter sets per generation). 


\subsection{Parameter fitting results}

Firstly, we see that the YFP clones in the $N o t c h 1^{+/+}$mice and the wild type intensity clones in the clonally induced $\mathrm{Notch}^{+/}$and Notch1\% mice have inferred fitness values closely centered on neutral (Extended data Fig. 4b, c, Table 1). Both Notch $1^{+/}$and Notch $1^{\%}$ clones are fitter than wild type clones, with Notch $1 \%$ clones substantially fitter than Notch $1^{+-}$clones. Should this be paralleled in humans, this large increase in fitness conveyed by the second Notch 1 mutation may explain the strong selection and high prevalence of "double hit" NOTCH1 mutant clones in aged human esophagus ${ }^{14}$ (Fig. 1c).

Table 1 Inferred fitness parameter values from $A B C$ fitting to lineage tracing data

\begin{tabular}{|l|l|l|l|}
\hline & Median inferred fitness & \multicolumn{2}{|l|}{$\begin{array}{l}95 \% \text { credible interval } \\
\text { Lower }\end{array}$} \\
\hline$+/+$ & 0.99 & 0.96 & Upper \\
\hline$+/+$ in $+/$ flox & 0.96 & 0.93 & 1.03 \\
\hline$+/+$ in flox/flox & 1.03 & 0.96 & 0.99 \\
\hline$+/-$ & 2.3 & 2.0 & 1.12 \\
\hline$-/-$ & 7.0 & 6.2 & 2.6 \\
\hline
\end{tabular}

In the cases of neutral clones (Notch $1^{+/+}$and wild type intensity clones from induced mutant mice) the induction proportion was not constrained by the fitting (Extended data Fig. 4b, Table 2), because these clones have the same fitness as the surrounding wild type cells and therefore do not impact the growth of any other clones. The inferred induction proportion for the Notch $1^{+/}$clones was larger than for the Notch1\% clones (Extended data Fig. 4b, Table 2). In simulations of the best fitting Notch1 ${ }^{+/}$ parameters, the mutant clones were colliding at the later time points, reducing the clone sizes (Video 1). This high density of clones and extensive clonal collision was not occurring to such an extent in the experimental data (Extended data Fig. 3g), suggesting that the simple model is not fully capturing all details of the Notch1+/- clonal dynamics and may be overestimating the fitness of large Notch $1^{+-}$ clones. However, the conclusions of the inferred fitness comparison are still valid: that the Notch $1^{+/-}$ clones have a clear growth advantage over wild type cells, and that the Notch $1^{-}$clones have much larger growth advantage than the Notch $1^{+/}$clones.

Table 2 Inferred induction proportion parameter values from $A B C$ fitting to lineage tracing data

\begin{tabular}{|l|l|l|l|}
\hline & Median inferred induction & \multicolumn{2}{|c|}{$95 \%$ credible interval } \\
Lower & Upper \\
\hline$+/+$ & 0.054 & 0.002 & 0.099 \\
\hline$+/+$ in +/flox & 0.051 & 0.004 & 0.098 \\
\hline$+/+$ in flox/flox & 0.052 & 0.003 & 0.097 \\
\hline$+/-$ & 0.023 & 0.017 & 0.029 \\
\hline$-/-$ & 0.0045 & 0.0001 & 0.0085 \\
\hline
\end{tabular}

\subsection{Simulations of haploinsufficiency}


It is clear from the lineage tracing experiments and the model fitting results that Notch1 is haploinsufficient, i.e. the heterozygous mutation gives a growth advantage to the clone. This growth advantage of the heterozygous mutant increases the chance of a double Notch1 mutant occurring because a higher proportion of the tissue becomes primed with the first mutation.

To illustrate the large difference haploinsufficiency makes, we ran simulations of haploinsufficient and haplosufficient versions of Notch1. In the haplosufficient simulations, the first Notch1 mutation has the same fitness as wild type cells, and a second Notch1 mutation in the same cell increases the fitness to the best fitting Notch- ${ }^{-/}$fitness (see section 1.4). In the haploinsufficient simulations, the first Notch1 mutation provides a fitness advantage equal to the best fitting Notch $1^{+/-}$fitness, then a second Notch1 mutation in the same cell increases the fitness to the best fitting Notch 1 - fitness

We ran simulations with a very low mutation rate to parallel the experiments in which we age wild type mice and observed the appearance of Notch1 mutant clones (Extended data Fig. 4e, f, Video 2). Each cell has a $0.0005 \%$ chance of gaining a mutation in each generation, with the mutation rate picked so that the haplosufficient simulations approximate the experimental data (Extended data Fig. 4e). In the simulations, a mutation can either appear on allele 1 or allele 2 of Notch 1 with $50 \%$ chance of each. A cell with a single mutant allele has the heterozygous fitness of that simulation type (see above), a cell with both alleles mutated has the homozygous fitness. A second mutation to the same allele does not change the cell fitness. We ran the simulations for 5000 days or until all cells in the tissue had both Notch1 alleles mutated.

The simulations showed that it would take substantially longer for the tissue to be swept by the homozygous mutant clones if Notch1 was haplosufficient (Extended data Fig. 4e, f, Video 2). This shows how the haploinsufficiency of Notch1 is a key property that allows the gene to dominate clonal competition in normal EE.

\section{DNA sequencing and coverage metrics}

\section{Custom baitset design}

For targeted sequencing experiments, we used three different Agilent SureSelect custom bait capture kits. Lists of the genes included in each custom bait capture kits are detailed in Supplementary table 1. For the Human dataset, we used a kit comprising 322 genes designed to include frequently mutated genes in cancer. For the targeted sequencing of mouse tissue, we used a kit comprising 73 cancer related and Notch pathway related genes. This kit (baitset_3181471_Covered.bed) also includes several regions of chromosome 2, which carries Notch1 gene. These regions, identified in a preliminary assay, allowed detection of SNP signal along the chromosome resulting in robust CNLOH analysis.

\section{$\underline{\text { Targeted sequencing }}$}

For targeted sequencing, gridded tissue and tumor samples (Methods) were submitted at standard input of 200 ng of DNA for library preparation. Human micro-dissected samples and mouse subclonal samples were submitted for DNA library preparation allowing a reduced DNA input from 100-1000 cells. Samples were multiplexed and sequenced on Illumina HiSeq 2000 sequencer using paired-end 75-base pair (bp) reads.

For gridded tissue and tumor mouse samples, the average coverage across all genes after removing off-target reads, PCR duplicates were: DEN-SOR Notch1 ${ }^{++}$grids: 478x; DEN-SOR tumors: $768 x$. 
For the samples submitted at low DNA input, the average coverage across all genes after removing off-target reads, PCR duplicates were: Human biopsies: 33x; Notch $1^{+/-}$subclonal biopsies: $194 x$.

\section{Alignment, variant calling and gene selection analysis}

\section{Alignment}

Paired-end reads were aligned with BWA-MEM (v.0.7.17, https://github.com/lh3/bwa) ${ }^{15}$ with optical and PCR duplicates marked using Biobambam2 (v.2.0.86, https://gitlab.com/german.tischler/biobambam2). Human samples were aligned to the GRCh37d5 reference genome whilst Mouse samples were mapped to the $\mathrm{GRCm} 38$ reference.

\section{-Mutation calling in the datasets}

Methods used for mutation calling were adapted to the dataset, depending on if the samples were clonal/near-clonal or if we aimed at identifying somatic mutations present in a small fraction of cells within the samples.

Human micro-dissected samples and mouse subclonal samples for clonal or nearly clonal (Fig. 1, Extended data Fig. 1, Fig. 3, Extended data fig. 5c-j, Supplementary tables 2, 4). Substitution mutations were called using the CaVEMan (Cancer Variants through Expectation Maximization, version 1.13.14) variant caller (http://cancerit.github.io/CaVEMan) ${ }^{16}$. Insertions and deletions were called using cgpPindel (http://cancerit.github.io/cgpPindel, version 3.3.0) ${ }^{17}$. Mutations were annotated using VAGrENT (https://github.com/cancerit/VAGrENT, version 3.3.3) ${ }^{18}$. Mutations occurring outside of the targeted genes were removed by retaining only mutations annotated with the effects 'missense', 'nonsense', 'ess_splice', 'frameshift', 'inframe' or 'silent'. The ShearwaterML pipeline (details below) was also used to analyze the Human dataset in order to report additional calling to the calls identified with CaVEMan and cgpPindel and allow a more exhaustive analysis of NOTCH1 mutations (Supplementary table 2).

Gridded normal tissue and tumors (Fig. 6, Extended data Fig. 7a,b, Supplementary table 7) were sampled to analyze gene selection, map somatic mutations clones spanning multiple samples or present in a small fraction of cells within the samples. To do so, we used ShearwaterML algorithm from the deepSNV package (v1.21.3, https://github.com/gerstung-lab/deepSNV) to call for mutation events on ultra-deep targeted data ${ }^{14,19,20}$. Instead of using a single-matched normal sample, the ShearwaterML algorithm uses a collection of deeply-sequenced normal samples as a reference for variant calling that enables the identification of mutations at very low allele frequencies.

The total coverage provided by the references used for each bait kits were as following. For the specifically designed Notch bait kit (Supplementary table 1), a total of 34 germline samples from the ears of the analyzed and the ears of extra mice providing a total coverage of $22885 x$. For the kit used for the Human sequencing, we used 68 germline samples for a total coverage of 14230x.

\section{$\underline{- \text { Gene selection }(d N d S)}$}

We used the maximum-likelihood implementation of the dNdScv algorithm (v0.0.1.0, https://github.com/im3sanger/dndscv) to identify genes under positive selection ${ }^{21}$. dNdScv estimates the ratio of non-synonymous to synonymous mutations across genes, controlling for the sequence 
composition of the gene and the mutational signatures, using trinucleotide context-dependent substitution matrices to avoid common mutation biases affecting $\mathrm{dN} / \mathrm{dS}$. Values of $\mathrm{dN} / \mathrm{dS}$ significantly higher than 1 indicate an excess of non-synonymous mutations in that particular gene and therefore imply positive selection, whereas $\mathrm{dN} / \mathrm{dS}$ values significantly lower than 1 suggest negative selection.

\section{-Mutant tissue coverage}

The size of mutant clones within each sample can be calculated by taking into account the area of the biopsy and the fraction of cells carrying a mutation within the sample, as described previously ${ }^{14,20}$. The lower (=VAF) and upper (=2xVAF) bound estimates of the percentage of epithelium covered by clones carrying non-synonymous mutations in a given gene was calculated for each biopsy. The fraction of epithelium covered by the mutant genes was then calculated from the mean of summed VAF (capped at 1.0) of all the biopsies in the same tissue.

\section{$\underline{- \text { Merging of clones }}$}

For analysis of the mutational landscape in the normal epithelium from highly mutagenized mice, we performed gridding of the tissue into $2 \mathrm{~mm}^{2}$ biopsies and kept record of the relative positions of the samples (Fig. 6b,c, Extended data Fig.7a). In order to avoid counting the same mutation multiple times and to obtain a more accurate estimate of clone sizes clonal mutations that spanned between two or more adjacent biopsies were merged as individual events ${ }^{14,20}$. To do this we calculated the mean number of shared mutations between biopsies at increasing distances, since the immediately adjacent samples are predicted to have more shared mutations than distant samples. Mutations common between samples closer than 3 mm were merged.

\section{Copy number variation and copy neutral loss of heterozygosity}

The NOTCH1 locus is frequently affected by copy neutral loss of heterozygosity (CNLOH) events ${ }^{14,20}$. CNLOH consists of the presence of two copies of either the maternal or paternal chromosome copy and none of the other. In sequencing data, this manifests itself as a change in the allele frequency of germline heterozygous SNPs (typically referred to as b-allele frequency, BAF), whilst the total coverage at the $\mathrm{CNLOH}$ event locus remains normal. The detection of a significant change in BAF and the absence of a change in coverage therefore constitutes $\mathrm{CNLOH}$. To detect these events, we applied two pipelines based on previously published methods: One to detect alterations in coverage data and one for germline heterozygous SNPs.

Whilst a change in total coverage can readily be detected in data from both human and mouse samples, typically, inbred mouse strains do not contain enough germline heterozygous SNPs for the purposes of detecting a BAF imbalance. This project, however, contains data sets from mice of mixedstrain background where we did detect the presence germline heterozygous SNPs. We reasoned that, because the mouse strains used in this project are common, any reliable SNP call from the Mouse Genome Project ${ }^{22}$, which is an effort to catalogue common strains, could be a viable germline heterozygous SNP candidate. Such a SNP would result from the mixture of strains, or rarely due to a de novo germline mutation. We therefore only considered SNPs that were reported by the Mouse 
Genome Project and subsequently applied stringent coverage criteria to identify heterozygous SNPs in the matched control samples during the analysis (which is detailed further below).

Since both coverage data and germline heterozygous SNPs are available for both species, we applied both pipelines to all human and mouse samples.

\subsection{Detection of changes in total copy number}

To call changes in total copy number in both human and mouse data we adapted QDNAseq for our purposes ${ }^{23}$. Briefly, QDNAseq typically collects read counts in bins across the genome, normalizes the counts to produce relative coverage (commonly referred to as logR; coverage log ratio), adjusts coverage for GC content correlated artifacts, segments the data in sections of constant signal and finally calls regions that are significantly different from normal as either a gain or a loss.

The standard QDNAseq pipeline was adapted in three ways. First, since the datasets described in this manuscript always include a matched control sample, we included an extra step in the pipeline to adjust for coverage variability observed in the control sample to further reduce noise, as described in 24.

Second, the calling of significantly different regions was adapted with a more conservative test, with the aim to produce robust calls. This step takes as input the segmented genome and each segment is tested by performing two one-sided t-tests: One to test for a gain and one to test for a loss. The test is performed between two distributions, one with the mean and standard deviation calculated from the $\log \mathrm{R}$ of the segment $(\mathrm{H} 1)$ and one with a mean of 0 (normal, unaltered $\log \mathrm{R}$ ) and the standard deviation of the segment ( $\mathrm{HO}$ ). This test may detect very small deviations for large segments, in-line with our aim for robust calls, we calculate the mean and standard deviation based on 100 sampled data bins for segments where more than 100 bins are available. The resulting $p$-values are adjusted for multiple testing (Bonferroni) and 0.05 is used as significance cutoff.

Finally, we included a pre-processing step to allow QDNAseq to run on targeted sequencing datasets. Typically, targeted sequencing data includes read-pairs that fall outside of the targeted genomic areas (referred to as off-target reads) and these off-target reads effectively form a shallow whole genome sequencing sample that can be used to call alterations ${ }^{25}$. To obtain this shallow genome we first count reads in $1 \mathrm{~kb}$ bins and subsequently remove any bin that overlaps with the targeted genomic coordinates. The remaining $1 \mathrm{~kb}$ bins are then mapped to $1 \mathrm{mb}$-sized bins, after which the regular pipeline is applied.

\subsection{Detection of allelic imbalance}

The pipeline to detect allelic imbalance in both human and mouse data through germline heterozygous SNPs builds on previously published methods ${ }^{14,24}$. This pipeline consists of 4 steps: (1) count reads for a set of reference SNPs (human: 1000 Genomes Project ${ }^{26}$ mouse: Mouse Genomes Project) and determine which SNPs are heterozygous based on the matched control sample. (2) Reconstruct haplotypes to improve the accuracy of the b-allele frequencies as is used in the Battenberg algorithm ${ }^{24}$, this step is skipped for mouse samples due to the requirement of reference 
haplotype data. (3) Segmentation of the data in regions of constant signal. (4) Calling of segments with significantly different signal and multiple-testing correction.

Allele counts are obtained for each potential SNP. For a read to be included in the count both the mapping quality and the base quality at the position within the read have to meet the below thresholds. A single read is counted for fragments where read pairs overlap, selecting the highest quality read to be included. These thresholds are applied to all counts in both the samples of interest and the matched controls.

\begin{tabular}{|l|l|l|}
\hline Description & Species & Value \\
\hline Minimum base quality & Both & 20 \\
\hline Minimum mapping quality & Both & 35 \\
\hline
\end{tabular}

A SNP was considered to be heterozygous when it met the criteria listed below in the matched germline sample. These thresholds aim to select bona fide heterozygous SNPs. Lower coverage thresholds were applied to the human samples due to their lower effective sequencing coverage. To compensate for the lower certainty in these samples, we subsequently increased the penalty of starting a new segment (segmentation Gamma).

\begin{tabular}{|l|l|l|}
\hline Description & Species & Value \\
\hline Minimum covering reads & Mouse & 25 \\
\hline Minimum covering reads & Human & 8 \\
\hline Minimum reads per allele & Mouse & 10 \\
\hline Minimum reads per allele & Human & 3 \\
\hline Minimum VAF & Both & 0.4 \\
\hline Maximum VAF & Both & 0.6 \\
\hline
\end{tabular}

For human samples we next perform haplotype reconstruction to refine the BAF values, as is implemented in the Battenberg algorithm. Segmentation is subsequently performed via piecewise constant fitting, as implemented in the ASCAT software package ${ }^{24}$, using the parameters listed below. This step results in segments that each have the following values: genomic start and end position, mean BAF in the sample of interest and mean BAF in the matched control.

\begin{tabular}{|l|l|l|}
\hline Description & Species & Value \\
\hline Gamma (reluctance to start a new segment) & Mouse & 6 \\
\hline Gamma & Human & 10 \\
\hline Kmin (minimum SNPs per segment) & Both & 3 \\
\hline
\end{tabular}

Finally, for each segment the BAF of the sample of interested is tested against the BAF of the matched control sample. The BAF is required to deviate 0.1 from 0.5 (i.e. BAF $<0.4$ or BAF $>0.6$ ) before a segment is tested, with the aim to prohibit noise from generating low quality calls. Testing is subsequently performed via a two-tailed t-test where the BAF of all heterozygous SNPs in the sample of interest are tested against the BAF of the same SNPs in the matched control. The resulting $p$-values 
are adjusted for multiple-testing (Bonferroni). A segment is considered having a BAF imbalance when the adjusted p-value from this test is below 0.05 .

\begin{tabular}{|l|l|l|}
\hline Description & Species & Value \\
\hline Minimum BAF deviation & Both & 0.1 \\
\hline T-test significance threshold & Both & 0.05 \\
\hline
\end{tabular}

\section{Single-cell RNA sequencing analysis}

\subsection{Alignment and transcript quantification}

Alignment of the sequencing reads and expression quantification was performed for each library individually using the CellRanger pipeline version 3.0.2 (10xGenomics). We subsequently used EmptyDrops version 1.2.2 ${ }^{27}$ to detect empty droplets in the raw feature count matrix output from CellRanger and discarded any barcode identified as an empty droplet. All the subsequent analysis described below was performed in R version 4.0.3 (https://www.R-project.org/ ) using the Seurat software package version $3.2 .0^{28}$.

\subsection{Cell type detection}

The pipeline to assign cell types first applies a number of filters per library to select healthy cells and remove any potential doublets. We filter on mitochondrial (MT) and overall expression levels: Cells are kept if their proportion of expression from MT genes is between 0.03 and 0.10 , total expressed genes is between 2,500 and 6,500 and total expression is below 55,000 total unique molecule identifiers (UMI). Genes are kept if they are expressed in 30 cells or more per library.

After filtering, expression is normalized and adjusted for proportion of MT expression, total expression, total number of expressed genes and predicted cell cycle state using the SCTransform function from Seurat. So far, all steps have been applied to the four libraries individually. The libraries are next combined using Seurat's SCT integration methodology that aims to incorporate libraries, taking into account possible batch effects. To capture the majority of the variation we required 3,000 genes to be used for the integration. Next, PCA was performed and we selected the top 30 principal components to continue into UMAP dimensionality reduction, clustering of cells and detection of variable genes between clusters. A plot of the UMAP space revealed good mixing of the four libraries throughout (Fig. 4g).

Cell types were identified through a series of marker genes. For keratinocytes we used Krt14, Tgm3, and Lor and required a per cell cluster average of at least 50, 5 and 10 transcripts per million (TPM) respectively (Extended data Fig. 6e). For fibroblasts we used Col1a1 with an average cluster TPM cutoff of greater than 20, for endothelial cells we used Pecam 1 requiring an average cluster TPM over 2 and, finally, for immune cells we used Cd83, Cd84, Cd86, Cd52 and Ptprc with average cluster TPM values of 1, 1, 0.4, 50 and 0.25 respectively. Extended data Fig. $6 c$ shows the expression of a number of selected markers overlaied on the UMAP space. Extended data Fig. 6e shows expression of a number of keratinocyte marker genes. 
Visual inspection of the assignment through a UMAP plot revealed that, even though the cell types were well separated (Fig. 4h, Supplementary table 6), 35 cells had been marked as keratinocytes, but visually clustered with the other cell types. These cells were flagged and their cell type label was set to NA to reflect their ambiguous status. These cells were not used for further analysis.

The number of cells per cell type, per library were counted and divided by the total number of cells per library to produce the data shown in Fig. $\mathbf{4 h}$.

\subsection{Analysis of keratinocytes}

Having identified which cells are keratinocytes, we restarted the analysis from the raw UMI counts, but now only including those cells that were previously marked as keratinocytes. The analysis differed from that above in two critical ways: 1 . No cell cycle adjustment was performed as the cell cycle state and keratinocyte differentiation are deeply linked, with basal cells cycling and differentiating cells having exited the cell cycle, and 2. as now only keratinocytes were included, we increased the minimum number of genes expressed per cell to 3,000 to strictly select for highly viable cells.

After integration of the four libraries, we performed a PCA and selected the top 10 principal components for further analysis. Clustering was performed and significant differentially expressed genes were identified through standard Seurat functions. Cells were assigned to a cell cycle phase using the Seurat function CellCycleScoring (Fig. 4j, Supplementary table 6) and we subsequently counted the cells per phase, per library and divided these counts by the total number of keratinocytes in each library to produce the data shown in Fig. $\mathbf{4 j}$.

To determine the number of basal cells per library we made use of the visual dividing line in the UMAP space marked by a clear drop in expression of Krt14 (basal cell marker gene) and the emergence of that of differentiation markers Krt4 and Tgm3 (Extended data Fig. 6e). The line separating the 'circle' shape from the 'tail' shape was defined to roughly capture the area where this expression divide manifests itself. We subsequently counted the cells from each library on both sides of the line to obtain the data shown in Fig. 4i (Supplementary table 6). We note that the exact placement of the dividing line matters little for the differences in proportions between libraries, because of the good mixing of cells from both wild-type and knock out libraries across the UMAP space (Fig. $\mathbf{4 g}$ ).

\section{Supplementary Note references}

1 Doupe, D. P. et al. A single progenitor population switches behavior to maintain and repair esophageal epithelium. Science (New York, N.Y.) 337, 1091-1093, (2012).

2 Clayton, E. et al. A single type of progenitor cell maintains normal epidermis. Nature 446, 185189, (2007).

3 Klein, A. M., Brash, D. E., Jones, P. H. \& Simons, B. D. Stochastic fate of $<$ em $>$ p53-</em>mutant epidermal progenitor cells is tilted toward proliferation by UV B during preneoplasia. Proceedings of the National Academy of Sciences 107, 270-275, (2010).

4 Murai, K. et al. Epidermal Tissue Adapts to Restrain Progenitors Carrying Clonal p53 Mutations. Cell stem cell 23, 687-699.e688, (2018). 

dynamics in normal epithelia. Journal of the Royal Society Interface 16, 20190230, (2019).

Colom, B. et al. Spatial competition shapes the dynamic mutational landscape of normal esophageal epithelium. Nature Genetics 52, 604-614, (2020).

Fowler, J. C. et al. Selection of oncogenic mutant clones in normal human skin varies with body site. Cancer Discovery, CD-20-1092, (2020).

Tavaré, S., Balding, D. J., Griffiths, R. C. \& Donnelly, P. Inferring coalescence times from DNA sequence data. Genetics 145, 505-518, (1997).

Beaumont, M. A., Zhang, W. \& Balding, D. J. Approximate Bayesian computation in population genetics. Genetics 162, 2025-2035, (2002).

11 Smirnov, N. V. Estimate of deviation between empirical distribution functions in two independent samples. Bull Moscow University 2, 3-16, (1939).

12 Toni, T., Welch, D., Strelkowa, N., Ipsen, A. \& Stumpf, M. P. Approximate Bayesian computation scheme for parameter inference and model selection in dynamical systems. Journal of the Royal Society, Interface 6, 187-202, (2009).

13 Klinger, E., Rickert, D. \& Hasenauer, J. pyABC: distributed, likelihood-free inference. Bioinformatics 34, 3591-3593, (2018).

14 Martincorena, I. et al. Somatic mutant clones colonize the human esophagus with age. Science (New York, N.Y.) 362, 911-917, (2018).

$15 \mathrm{Li}, \mathrm{H}$. Aligning sequence reads, clone sequences and assembly contigs with BWA-MEM. ArXiv, 1303.3997, (2013).

16 Jones, D. et al. cgpCaVEManWrapper: Simple Execution of CaVEMan in Order to Detect Somatic Single Nucleotide Variants in NGS Data. Current Protocols in Bioinformatics 56, 15.10.11-15.10.18, (2016).

17 Raine, K. M. et al. cgpPindel: Identifying Somatically Acquired Insertion and Deletion Events from Paired End Sequencing. Current protocols in bioinformatics 52, 15.17.11-15.17.12 (2015).

18 Menzies, A. et al. VAGrENT: Variation Annotation Generator. Current Protocols in Bioinformatics 52, 15.18.11-15.18.11, (2015).

19 Gerstung, M., Papaemmanuil, E. \& Campbell, P. J. Subclonal variant calling with multiple samples and prior knowledge. Bioinformatics 30, 1198-1204, (2014).

20 Martincorena, I. et al. Tumor evolution. High burden and pervasive positive selection of somatic mutations in normal human skin. Science (New York, N.Y.) 348, 880-886, (2015).

21 Martincorena, I. et al. Universal Patterns of Selection in Cancer and Somatic Tissues. Cell 171, 1029-1041.e1021, (2017).

22 Keane, T. M. et al. Mouse genomic variation and its effect on phenotypes and gene regulation. Nature 477, 289-294, (2011). 

made available under aCC-BY 4.0 International license.

23 Scheinin, I. et al. DNA copy number analysis of fresh and formalin-fixed specimens by shallow whole-genome sequencing with identification and exclusion of problematic regions in the genome assembly. Genome research 24, 2022-2032, (2014).

24 Nik-Zainal, S. et al. The life history of 21 breast cancers. Cell 149, 994-1007, (2012).

25 Kuilman, T. et al. CopywriteR: DNA copy number detection from off-target sequence data. Genome biology 16, 49, (2015).

26 McVean, G. A. et al. An integrated map of genetic variation from 1,092 human genomes. Nature 491, 56-65, (2012).

27 Lun, A. T. L. et al. EmptyDrops: distinguishing cells from empty droplets in droplet-based single-cell RNA sequencing data. Genome biology 20, 63, (2019).

28 Stuart, T. et al. Comprehensive Integration of Single-Cell Data. Cell 177, 1888-1902.e1821, (2019). 